\title{
Ancient solutions to the Ricci flow in dimension 3
}

\author{
by \\ Simon BRENDLE \\ Columbia University \\ New York, NY, U.S.A.
}

\section{Contents}

1. Introduction . . . . . . . . . . . . . . . . . . . 1

Part I. Proof of Theorem $1.1 \ldots \ldots \ldots \ldots$

2. A barrier construction . . . . . . . . . . . . . . 4

3. Asymptotics of ancient $\kappa$-solutions with rotational symmetry . . . 15

4. Uniqueness of ancient $\kappa$-solutions with rotational symmetry . . . 27

Part II. Proof of Theorem $1.2 \ldots \ldots \ldots 42$

5. A PDE for the Lie derivative of the metric along a vector field . . . 42

6. The parabolic Lichnerowicz equation on shrinking cylinders . . . . . 44

7. Gluing approximate Killing vector fields . . . . . . . . . . . . . 53

8. The neck improvement theorem . . . . . . . . . . . 58

9. Rotational symmetry of ancient $\kappa$-solutions in dimension $3 \ldots 75$

Appendix A. Summary of known results about ancient $\kappa$-solutions . . 95

Appendix B. A variant of the Anderson-Chow estimate . . . . . . . . 99

References ... . . . . . . . . . . . . . . . . . . 101

\section{Introduction}

A central problem in the study of geometric flows is to understand singularity formation. It turns out that singularities can often be modeled on ancient solutions; these are solutions which are defined on $(-\infty, T]$. The notion of an ancient solution was first introduced in Hamilton's work [17]. Perelman [21] studied ancient solutions to the Ricci flow in dimension 3 which are complete; non-flat; $\kappa$-non-collapsed; and have bounded and non-negative curvature. These solutions are referred to as ancient $\kappa$-solutions. Perelman [21] proved that every finite-time singularity of the Ricci flow in dimension 3 is modeled on an ancient $\kappa$-solution. Moreover, he proved an important structure theorem for ancient $\kappa$-solutions. Roughly speaking, this theorem asserts that every non-compact 
ancient $\kappa$-solution with positive sectional curvature consists of a tube with a positively curved cap attached on one side.

The purpose of this paper is to give a classification of all non-compact ancient $\kappa$ solutions in dimension 3. In the first part of this paper, we classify all non-compact ancient $\kappa$-solutions with rotational symmetry.

Theorem 1.1. Assume that $(M, g(t))$ is a 3-dimensional ancient $\kappa$-solution which is non-compact and has positive sectional curvature. If $(M, g(t))$ is rotationally symmetric, then $(M, g(t))$ is isometric to the Bryant soliton up to scaling.

In the second part of this paper, we reduce the classification of non-compact ancient $\kappa$-solutions to the rotationally symmetric case.

Theorem 1.2. Assume that $(M, g(t))$ is a 3-dimensional ancient $\kappa$-solution which is non-compact and has positive sectional curvature. Then $(M, g(t))$ is rotationally symmetric.

Theorem 1.2 extends our earlier work [8], where we proved that the Bryant soliton is the only non-collapsed steady gradient Ricci soliton in dimension 3 . Note that, by work of Chen, every complete ancient solution to the Ricci flow in dimension 3 has non-negative sectional curvature (see [11, Corollary 2.4]).

Combining Theorems 1.1 and 1.2, we obtain the following result.

Theorem 1.3. Assume that $(M, g(t))$ is a 3-dimensional ancient $\kappa$-solution which is non-compact. Then $(M, g(t))$ is isometric to either a family of shrinking cylinders (or a quotient thereof), or to the Bryant soliton.

Combining Theorem 1.3 with work of Perelman [21], we can draw the following conclusion.

Corollary 1.4. Consider a solution to the Ricci flow on a compact 3-manifold which forms a singularity in a finite time. Then, at the first singular time, the only possible blow-up limits are quotients of the round sphere $S^{3}$, quotients of the standard cylinder $S^{2} \times \mathbb{R}$, and the Bryant soliton.

Let us sketch how Corollary 1.4 follows from Theorem 1.3. Consider a smooth solution of the Ricci flow on a compact 3-manifold which is defined on a finite-time interval $[0, T)$ and becomes singular as $t \rightarrow T$. By work of Perelman [21], every blowup limit as $t \rightarrow T$ is an ancient $\kappa$-solution. If a blow-up limit is compact with strictly positive sectional curvature, then the original flow will have positive sectional curvature for $t$ sufficiently close to $T$. A classical theorem of Hamilton [14] then implies that the original flow becomes round as $t \rightarrow T$. If a blow-up limit is non-compact with strictly 
positive sectional curvature, then it must be the Bryant soliton by Theorem 1.3. Finally, if a blow-up limit does not have strictly positive sectional curvature, then it must be a quotient of the cylinder by standard isometries.

Let us mention some related work. In [13], Daskalopoulos, Hamilton, and Šešum obtained a classification of all compact ancient solutions to the Ricci flow in dimension 2. In [9], it was shown that the bowl soliton is the only non-compact ancient solution to the mean curvature flow in $\mathbb{R}^{3}$ which is non-collapsed and strictly convex. Angenent, Daskalopoulos, and Šešum [3] later obtained a classification of all compact ancient solutions to mean curvature flow in $\mathbb{R}^{3}$ which are non-collapsed and strictly convex.

We now give an overview of the main ideas involved in the proof of Theorems 1.1 and 1.2 .

In the first part of this paper, we classify non-compact ancient $\kappa$-solutions with rotational symmetry. In $\S 2$, we set up a barrier argument for solutions to the Ricci flow with rotational symmetry. One important ingredient in our barrier construction are the steady gradient Ricci solitons with singularity at the tip which were found by Robert Bryant [10]. In $\S 3$, we study the asymptotic behavior of a non-compact ancient $\kappa$-solution with rotational symmetry. To that end, we focus on the cylindrical region, and carry out a spectral decomposition in Hermite polynomials. As in [4] and [9], a subtle point here is that we need to control certain error terms arising from the cut-off functions. In our work, this is done using barrier arguments. Using the spectral analysis, we obtain precise asymptotics for the solution in the cylindrical region. Combining these estimates with the barrier arguments, we conclude that $\liminf _{t \rightarrow-\infty} R_{\max }(t)>0$ (see Proposition 3.17). In $\S 4$, we complete the proof of Theorem 1.1. The idea is to consider a quantity which is constant on the Bryant soliton, and then show that this quantity must be constant on any non-compact ancient $\kappa$-solution with rotational symmetry.

In the second part of this paper, we show that every non-compact ancient $\kappa$-solution must be rotationally symmetric. In $\S 5$, we derive a crucial evolution equation for the Lie derivative of the metric along a vector field. In $\S \S 6-8$, we establish a neck improvement theorem for the Ricci flow, which tells us that a neck-like region becomes more symmetric under the evolution. The proof of the neck improvement theorem is based on the vector field method developed in [8], and requires a careful analysis of the parabolic Lichnerowicz equation on the cylinder. Finally, in $\S 9$, we complete the proof of Theorem 1.2. The idea is as follows. Since our solution is of type II, we can find a sequence of points $\left(\hat{p}_{k}, \hat{t}_{k}\right)$ in space-time such that, if we rescale the flow around $\left(\hat{p}_{k}, \hat{t}_{k}\right)$, then the rescaled flows converge to a steady gradient Ricci soliton as $k \rightarrow \infty$. By [8], this limiting soliton must be the Bryant soliton. In particular, we can find a sequence $\hat{\varepsilon}_{k} \rightarrow 0$ such that the flow is $\hat{\varepsilon}_{k^{-}}$ symmetric at time $\hat{t}_{k}$ (see Definition 9.2 for a precise definition). We now move forward 
in time, starting from time $\hat{t}_{k}$. As long as the solution is nearly rotationally symmetric, it will remain close to the Bryant soliton by Theorem 1.1. On the other hand, as long as the cap is close to the Bryant soliton, we are able to show that the symmetry improves under the evolution (see Proposition 9.19). Using a continuity argument, we are able to show that there exists a sequence $\varepsilon_{k} \geqslant 2 \hat{\varepsilon}_{k}$ such that $\varepsilon_{k} \rightarrow 0$ and the flow is $\varepsilon_{k}$-symmetric at time $t$ for all $t \in\left[\hat{t}_{k}, 0\right]$. Passing to the limit as $k \rightarrow \infty$, it follows that $(M, g(t))$ is rotationally symmetric for all $t$.

Remark 1.5. The proof of Theorem 1.2 can be adapted to the compact case. This will imply that every ancient $\kappa$-solution on $S^{3}$ must be rotationally symmetric.

Acknowledgements. I am grateful to Robert Bryant for sharing with me his insights on singular Ricci solitons (cf. Theorem 2.1), and to Kyeongsu Choi for pointing out to me his variant of the Anderson-Chow estimate (cf. Theorem B.1). I would like to thank Keaton Naff for comments on an earlier version of this paper. I am grateful to Tübingen University, where part of this work was carried out. This project was supported by the National Science Foundation under grant DMS-1806190 and by the Simons Foundation.

\section{Part I. Proof of Theorem 1.1}

\section{A barrier construction}

In this section, we study the Ricci flow in the rotationally symmetric setting. In this case, the Ricci flow reduces to a parabolic equation for a single scalar function (see [6]). We first construct a family of functions $\psi_{a}$ which will serve as barriers. A key ingredient in our construction is the following result due to Robert Bryant [10] (see also [1, Proposition 2.1]).

TheOREm 2.1. (Bryant $[10, \S 3.4])$ There exists a steady gradient Ricci soliton which is rotationally symmetric, singular at the tip, and asymptotic to the Bryant soliton near infinity. This soliton can be written in the form $\varphi(r)^{-1} d r \otimes d r+r^{2} g_{S^{2}}$, where $\varphi(r)$ is a positive function defined on the interval $(0, \infty)$ satisfying

$$
\varphi(r) \varphi^{\prime \prime}(r)-\frac{1}{2} \varphi^{\prime}(r)^{2}+r^{-2}(1-\varphi(r))\left(r \varphi^{\prime}(r)+2 \varphi(r)\right)=0 .
$$

The function $\varphi(r)$ satisfies $\varphi(r) \rightarrow \infty$ as $r \rightarrow 0$. Like the Bryant soliton, $\varphi(r)$ satisfies an asymptotic expansion of the form $\varphi(r)=r^{-2}+2 r^{-4}+O\left(r^{-6}\right)$ as $r \rightarrow \infty$.

Proof. We sketch how Theorem 2.1 follows from Bryant's results. In equation (3.26) in [10], Robert Bryant considers the ordinary differential equation (ODE)

$$
\frac{d u}{d s}=\frac{u\left(1-u^{2}\right) s^{2}}{(u+s)\left(2-s^{2}\right)}
$$


It is shown in [10] that this ODE admits a solution $u(s)$ which is defined for $s \in(-\sqrt{2}, 0)$, takes values in the interval $(0,1)$, and satisfies $u(s) \rightarrow 1$ as $s \searrow-\sqrt{2}$ and $u(s) \rightarrow 0$ as $s \nearrow 0$. Moreover, this solution satisfies $u(s)+s<0$ for all $s \in(-\sqrt{2}, 0)$. Given a solution $u(s)$ of this ODE, the metric

$$
g=\frac{1-u^{2}}{(u+s)^{2}\left(2-s^{2}\right)^{2}} d s \otimes d s+\frac{1-u^{2}}{u^{2}\left(2-s^{2}\right)} g_{S^{2}}
$$

will be a steady gradient Ricci soliton (cf. equation (3.28) in [10]). Using the differential equation for $u$, we compute

$$
\frac{1}{2} \frac{d}{d s}\left(\frac{1-u^{2}}{u^{2}\left(2-s^{2}\right)}\right)=\frac{\left(1-u^{2}\right) s}{u(u+s)\left(2-s^{2}\right)^{2}}>0
$$

for all $s \in(-\sqrt{2}, 0)$. Consequently, the function $s \mapsto\left(1-u^{2}\right) / u^{2}\left(2-s^{2}\right)$ is strictly monotone increasing. Moreover, $\left(1-u^{2}\right) / u^{2}\left(2-s^{2}\right) \rightarrow 0$ as $s \searrow-\sqrt{2}$, and $\left(1-u^{2}\right) / u^{2}\left(2-s^{2}\right) \rightarrow \infty$ as $s \nearrow 0$. Hence, the metric $g$ can be rewritten as

$$
g=\varphi(r)^{-1} d r \otimes d r+r^{2} g_{S^{2}}
$$

where $\varphi$ is defined by

$$
\varphi\left(\sqrt{\frac{1-u^{2}}{u^{2}\left(2-s^{2}\right)}}\right)=\frac{s^{2}}{2-s^{2}} .
$$

The function $\varphi(r)$ is defined for all $r \in(0, \infty)$, and satisfies the ODE

$$
\varphi(r) \varphi^{\prime \prime}(r)-\frac{1}{2} \varphi^{\prime}(r)^{2}+r^{-2}(1-\varphi(r))\left(r \varphi^{\prime}(r)+2 \varphi(r)\right)=0 .
$$

Moreover, $\varphi(r) \rightarrow \infty$ as $r \rightarrow 0$. Finally, after replacing $\varphi(r)$ by $\varphi(c r)$ for a suitable constant $c>0$, the function $\varphi(r)$ will have the desired asymptotic expansion as $r \rightarrow \infty$. From this, Theorem 2.1 follows.

Remark 2.2. Robert Bryant proved that there is a 1-parameter family of singular steady gradient Ricci solitons, which satisfy $\varphi(r) \sim r^{-2(\sqrt{2}-1)}$ as $r \rightarrow 0$. However, for the purposes of this paper, one example is sufficient.

In the following, we fix a function $\varphi$ as in Theorem 2.1. Moreover, we fix a positive number $r_{*}$ such that $\varphi\left(r_{*}\right)=2$.

Let us choose a smooth function $\zeta$ such that

$$
\frac{d}{d s}\left(\left(s^{-2}-1\right)^{-1} \zeta(s)\right)=\left(s^{-2}-1\right)^{-2}\left(2 s^{-3}-5 s^{-6}-\frac{1}{2} s^{27}\right) .
$$

Note that

$$
\left(s^{-2}-1\right)^{-2}\left(2 s^{-3}-5 s^{-6}-\frac{1}{2} s^{27}\right)=-5 s^{-2}+O(1)
$$


as $s \rightarrow 0$, and

$$
\left(s^{-2}-1\right)^{-2}\left(2 s^{-3}-5 s^{-6}-\frac{1}{2} s^{27}\right)=-\frac{7}{8}(1-s)^{-2}+O(1) .
$$

as $s \rightarrow 1$. The first statement gives $\zeta(s)=5 s^{-3}+O\left(s^{-2}\right)$ as $s \rightarrow 0$. The second statement implies that $\zeta(s)$ is indeed smooth at $s=1$, and $\zeta(1)=-\frac{7}{4}$. By continuity, we can find a small constant $\theta \in\left(0, \frac{1}{100}\right)$ such that $2 s^{-4}+\zeta(s) \geqslant \frac{1}{8}$ for all $s \in[1-\theta, 1+\theta]$.

LEMma 2.3. We can find a large constant $N$ with the following property. If a is sufficiently large, then

$$
\varphi(a s)-a^{-2}+a^{-4} \zeta(s) \geqslant a^{-2}\left(s^{-2}-1\right)+\frac{1}{16} a^{-4}
$$

for all $s \in\left[1-\theta, 1+\frac{1}{100} a^{-2}\right]$, and

$$
\varphi(a s)-a^{-2}+a^{-4} \zeta(s) \geqslant \frac{1}{32} a^{-4}
$$

for all $s \in\left[N a^{-1}, 1+\frac{1}{100} a^{-2}\right]$.

Proof. Since $2 s^{-4}+\zeta(s) \geqslant \frac{1}{8}$ for all $s \in[1-\theta, 1+\theta]$, we obtain

$$
\begin{aligned}
\varphi(a s)-a^{-2}+a^{-4} \zeta(s) & =a^{-2}\left(s^{-2}-1\right)+a^{-4}\left(2 s^{-4}+\zeta(s)\right)+O\left(a^{-6}\right) \\
& \geqslant a^{-2}\left(s^{-2}-1\right)+\frac{1}{8} a^{-4}+O\left(a^{-6}\right)
\end{aligned}
$$

for all $s \in[1-\theta, 1+\theta]$. Consequently, if $a$ is sufficiently large, then

$$
\varphi(a s)-a^{-2}+a^{-4} \zeta(s) \geqslant a^{-2}\left(s^{-2}-1\right)+\frac{1}{16} a^{-4}
$$

for all $s \in[1-\theta, 1+\theta]$. This proves the first statement.

In particular, if $a$ is sufficiently large, then

$$
\varphi(a s)-a^{-2}+a^{-4} \zeta(s) \geqslant \frac{1}{32} a^{-4}
$$

for all $s \in\left[1-\theta, 1+\frac{1}{100} a^{-2}\right]$. We next observe that

$$
\varphi(a s)-a^{-2}+a^{-4} \zeta(s)=a^{-2} s^{-2}-a^{-2}+O\left(a^{-4} s^{-4}\right)
$$

for all $s \in\left[r_{*} a^{-1}, 1-\theta\right]$. Hence, if we choose $N$ sufficiently large (depending on $\theta$ ), then

$$
\varphi(a s)-a^{-2}+a^{-4} \zeta(s) \geqslant(1-\theta) a^{-2} s^{-2}-a^{-2} \geqslant\left((1-\theta)^{-1}-1\right) a^{-2}
$$

for all $s \in\left[N a^{-1}, 1-\theta\right]$. In particular, if $a$ is sufficiently large (depending on $\theta$ ), then

$$
\varphi(a s)-a^{-2}+a^{-4} \zeta(s) \geqslant \frac{1}{32} a^{-4}
$$

for all $s \in\left[N a^{-1}, 1-\theta\right]$. Putting these facts together, the second statement follows. The proof of Lemma 2.3 is now complete. 
LEMma 2.4. We can find a large constant $N$ with the following property. Suppose that a is sufficiently large, and let

$$
\psi_{a}(s):=\varphi(a s)-a^{-2}+a^{-4} \zeta(s)
$$

for $s \in\left[N a^{-1}, 1+\frac{1}{100} a^{-2}\right]$. Then,

$$
\psi_{a}(s) \psi_{a}^{\prime \prime}(s)-\frac{1}{2} \psi_{a}^{\prime}(s)^{2}+s^{-2}\left(1-\psi_{a}(s)\right)\left(s \psi_{a}^{\prime}(s)+2 \psi_{a}(s)\right)-s \psi_{a}^{\prime}(s)<0
$$

for $s \in\left[N a^{-1}, 1+\frac{1}{100} a^{-2}\right]$.

Proof. The function $\zeta$ satisfies

$$
\begin{aligned}
s^{-2}\left(s \zeta^{\prime}(s)+2 \zeta(s)\right)-s \zeta^{\prime}(s) & =s\left(s^{-2}-1\right)^{2} \frac{d}{d s}\left(\left(s^{-2}-1\right)^{-1} \zeta(s)\right) \\
& =2 s^{-2}-5 s^{-5}-\frac{1}{2} s^{28}
\end{aligned}
$$

Since $-r \varphi^{\prime}(r)-2 r^{-2}=8 r^{-4}+O\left(r^{-6}\right)$ as $r \rightarrow \infty$, it follows that

$$
\begin{aligned}
s^{-2}\left(s \psi_{a}^{\prime}(s)+2 \psi_{a}(s)\right)-s \psi_{a}^{\prime}(s)= & s^{-2}\left(a s \varphi^{\prime}(a s)+2 \varphi(a s)\right)-a s \varphi^{\prime}(a s)-2 a^{-2} s^{-2} \\
& +2 a^{-4} s^{-2}-5 a^{-4} s^{-5}-\frac{1}{2} a^{-4} s^{28} \\
=s^{-2}\left(a s \varphi^{\prime}(a s)+2 \varphi(a s)\right)+8 a^{-4} s^{-4} & +2 a^{-4} s^{-2}-5 a^{-4} s^{-5}-\frac{1}{2} a^{-4} s^{28}+O\left(a^{-6} s^{-6}\right)
\end{aligned}
$$

for $s \in\left[r_{*} a^{-1}, 1+\frac{1}{100} a^{-2}\right]$. Moreover, using the identity

$$
\varphi^{\prime \prime}(r)-r^{-2}\left(r \varphi^{\prime}(r)+4 \varphi(r)\right)=4 r^{-4}+O\left(r^{-6}\right)
$$

as $r \rightarrow \infty$, we obtain

$$
\begin{gathered}
\psi_{a}(s) \psi_{a}^{\prime \prime}(s)-\frac{1}{2} \psi_{a}^{\prime}(s)^{2}-s^{-2} \psi_{a}(s)\left(s \psi_{a}^{\prime}(s)+2 \psi_{a}(s)\right) \\
=a^{2} \varphi(a s) \varphi^{\prime \prime}(a s)-\frac{1}{2} a^{2} \varphi^{\prime}(a s)^{2}-s^{-2} \varphi(a s)\left(a s \varphi^{\prime}(s)+2 \varphi(a s)\right) \\
-2 a^{-4} s^{-2}-\left[\varphi^{\prime \prime}(a s)-a^{-2} s^{-2}\left(a s \varphi^{\prime}(a s)+4 \varphi(a s)\right)\right] \\
+a^{-4}\left[\varphi(a s) \zeta^{\prime \prime}(s)+a^{2} \varphi^{\prime \prime}(a s) \zeta(s)-a \varphi^{\prime}(a s) \zeta^{\prime}(s)\right. \\
\left.-s^{-1} \varphi(a s) \zeta^{\prime}(s)-a s^{-1} \varphi^{\prime}(a s) \zeta(s)-4 s^{-2} \varphi(a s) \zeta(s)\right] \\
-a^{-6}\left[\zeta^{\prime \prime}(s)-s^{-2}\left(s \zeta^{\prime}(s)+4 \zeta(s)\right)\right] \\
+a^{-8}\left[\zeta(s) \zeta^{\prime \prime}(s)-\frac{1}{2} \zeta^{\prime}(s)^{2}-s^{-2} \zeta(s)\left(s \zeta^{\prime}(s)+2 \zeta(s)\right)\right] \\
=a^{2} \varphi(a s) \varphi^{\prime \prime}(a s)-\frac{1}{2} a^{2} \varphi^{\prime}(a s)^{2}-s^{-2} \varphi(a s)\left(a s \varphi^{\prime}(s)+2 \varphi(a s)\right) \\
-2 a^{-4} s^{-2}-4 a^{-4} s^{-4}+O\left(a^{-6} s^{-7}\right)
\end{gathered}
$$


for $s \in\left[r_{*} a^{-1}, 1+\frac{1}{100} a^{-2}\right]$. Adding both identities yields

$$
\begin{aligned}
\psi_{a}(s) \psi_{a}^{\prime \prime}(s) & -\frac{1}{2} \psi_{a}^{\prime}(s)^{2}+s^{-2}\left(1-\psi_{a}(s)\right)\left(s \psi_{a}^{\prime}(s)+2 \psi_{a}(s)\right)-s \psi_{a}^{\prime}(s) \\
= & a^{2}\left[\varphi(a s) \varphi^{\prime \prime}(a s)-\frac{1}{2} \varphi^{\prime}(a s)^{2}+(a s)^{-2}(1-\varphi(a s))\left(a s \varphi^{\prime}(s)+2 \varphi(a s)\right)\right] \\
& +4 a^{-4} s^{-4}-5 a^{-4} s^{-5}-\frac{1}{2} a^{-4} s^{28}+O\left(a^{-6} s^{-7}\right)
\end{aligned}
$$

for $s \in\left[r_{*} a^{-1}, 1+\frac{1}{100} a^{-2}\right]$. Using the ODE for $\varphi(r)$, we conclude that

$$
\begin{gathered}
\psi_{a}(s) \psi_{a}^{\prime \prime}(s)-\frac{1}{2} \psi_{a}^{\prime}(s)^{2}+s^{-2}\left(1-\psi_{a}(s)\right)\left(s \psi_{a}^{\prime}(s)+2 \psi_{a}(s)\right)-s \psi_{a}^{\prime}(s) \\
=4 a^{-4} s^{-4}-5 a^{-4} s^{-5}-\frac{1}{2} a^{-4} s^{28}+O\left(a^{-6} s^{-7}\right)
\end{gathered}
$$

for $s \in\left[r_{*} a^{-1}, 1+\frac{1}{100} a^{-2}\right]$. Clearly, the expression on the right-hand side is negative if $s \in\left[N a^{-1}, 1+\frac{1}{100} a^{-2}\right]$ and $N$ is sufficiently large.

From now on, we will fix a large number $N$ so that the conclusions of Lemmas 2.3 and 2.4 hold. For $a$ sufficiently large, we can find a smooth function $\beta_{a}(r)$ such that

$$
\begin{aligned}
& \beta_{a}(N)=a^{-3} \zeta\left(N a^{-1}\right)-a^{-1}, \\
& \beta_{a}^{\prime}(N)=a^{-4} \zeta^{\prime}\left(N a^{-1}\right),
\end{aligned}
$$

and

$$
\begin{aligned}
\varphi(r) & \beta_{a}^{\prime \prime}(r)+\varphi^{\prime \prime}(r) \beta_{a}(r)-\varphi^{\prime}(r) \beta_{a}^{\prime}(r) \\
& +r^{-2}(1-\varphi(r))\left(r \beta_{a}^{\prime}(r)+2 \beta_{a}(r)\right)-r^{-2} \beta_{a}(r)\left(r \varphi^{\prime}(r)+2 \varphi(r)\right)=-1
\end{aligned}
$$

for $r \in\left[r_{*}, N\right]$. Note that $\beta_{a}(N)$ and $\beta_{a}^{\prime}(N)$ are uniformly bounded independent of $a$. Consequently, the function $\beta_{a}$ and all its derivatives are uniformly bounded on the interval $\left[r_{*}, N\right]$, and the bounds are independent of $a$.

Lemma 2.5. Suppose that a is sufficiently large, and let

$$
\psi_{a}(s):=\varphi(a s)+a^{-1} \beta_{a}(a s)
$$

for $s \in\left[r_{*} a^{-1}, N a^{-1}\right]$. Then,

$$
\psi_{a}(s) \psi_{a}^{\prime \prime}(s)-\frac{1}{2} \psi_{a}^{\prime}(s)^{2}+s^{-2}\left(1-\psi_{a}(s)\right)\left(s \psi_{a}^{\prime}(s)+2 \psi_{a}(s)\right)-s \psi_{a}^{\prime}(s)<0
$$

for all $s \in\left[r_{*} a^{-1}, N a^{-1}\right]$. 
Proof. Using the ODEs for $\varphi(r)$ and $\beta_{a}(r)$, we obtain

$$
\begin{gathered}
\psi_{a}(s) \psi_{a}^{\prime \prime}(s)-\frac{1}{2} \psi_{a}^{\prime}(s)^{2}+s^{-2}\left(1-\psi_{a}(s)\right)\left(s \psi_{a}^{\prime}(s)+2 \psi_{a}(s)\right) \\
=a^{2}\left[\varphi(a s) \varphi^{\prime \prime}(a s)-\frac{1}{2} \varphi^{\prime}(a s)^{2}+(a s)^{-2}(1-\varphi(a s))\left(a s \varphi^{\prime}(a s)+2 \varphi(a s)\right)\right] \\
+a\left[\varphi(a s) \beta_{a}^{\prime \prime}(a s)+\varphi^{\prime \prime}(a s) \beta_{a}(a s)-\varphi^{\prime}(a s) \beta_{a}^{\prime}(a s)\right. \\
+(a s)^{-2}(1-\varphi(a s))\left(a s \beta_{a}^{\prime}(a s)+2 \beta_{a}(a s)\right) \\
\left.\quad-(a s)^{-2} \beta_{a}(a s)\left(a s \varphi^{\prime}(a s)+2 \varphi(a s)\right)\right] \\
+\left[\beta_{a}(a s) \beta_{a}^{\prime \prime}(a s)-\frac{1}{2} \beta_{a}^{\prime}(a s)^{2}-(a s)^{-2} \beta_{a}(a s)\left(a s \beta_{a}^{\prime}(a s)+2 \beta_{a}(a s)\right)\right] \\
\leqslant-a+C
\end{gathered}
$$

for all $s \in\left[r_{*} a^{-1}, N a^{-1}\right]$. On the other hand,

$$
s \psi_{a}^{\prime}(s)=a s \varphi^{\prime}(a s)+s \beta_{a}^{\prime}(a s) \geqslant-C
$$

for all $s \in\left[r_{*} a^{-1}, N a^{-1}\right]$. Hence, if $a$ is sufficiently large, then

$$
\psi_{a}(s) \psi_{a}^{\prime \prime}(s)-\frac{1}{2} \psi_{a}^{\prime}(s)^{2}+s^{-2}\left(1-\psi_{a}(s)\right)\left(s \psi_{a}^{\prime}(s)+2 \psi_{a}(s)\right)<s \psi_{a}^{\prime}(s)
$$

for all $s \in\left[r_{*} a^{-1}, N a^{-1}\right]$.

After these preparations, we now give the definition of our barriers:

Definition 2.6. Suppose that $a$ is sufficiently large. We define a function

$$
\psi_{a}:\left[r_{*} a^{-1}, 1+\frac{1}{100} a^{-2}\right] \longrightarrow \mathbb{R}
$$

by

$$
\psi_{a}(s):= \begin{cases}\varphi(a s)-a^{-2}+a^{-4} \zeta(s) & \text { for } s \in\left[N a^{-1}, 1+\frac{1}{100} a^{-2}\right], \\ \varphi(a s)+a^{-1} \beta_{a}(a s), & \text { for } s \in\left[r_{*} a^{-1}, N a^{-1}\right] .\end{cases}
$$

Using Lemmas 2.3-2.5, we can draw the following conclusion:

Proposition 2.7. Suppose that a is sufficiently large. Then, $\psi_{a}$ is continuously differentiable, and

$$
\psi_{a}(s) \psi_{a}^{\prime \prime}(s)-\frac{1}{2} \psi_{a}^{\prime}(s)^{2}+s^{-2}\left(1-\psi_{a}(s)\right)\left(s \psi_{a}^{\prime}(s)+2 \psi_{a}(s)\right)-s \psi_{a}^{\prime}(s)<0
$$

for all $s \in\left[r_{*} a^{-1}, 1+\frac{1}{100} a^{-2}\right]$. Also, we have $\psi_{a}(s) \geqslant \frac{1}{32} a^{-4}$ for all $s \in\left[r_{*} a^{-1}, 1+\frac{1}{100} a^{-2}\right]$, and $\psi_{a}(s) \geqslant a^{-2}\left(s^{-2}-1\right)+\frac{1}{16} a^{-4}$ for all $s \in\left[1-\theta, 1+\frac{1}{100} a^{-2}\right]$. 
Proof. Recall that $\beta_{a}(N)=a^{-3} \zeta\left(N a^{-1}\right)-a^{-1}$ and $\beta_{a}^{\prime}(N)=a^{-4} \zeta^{\prime}\left(N a^{-1}\right)$. This implies that $\psi_{a}$ is continuously differentiable at the point $s=N a^{-1}$. This proves the first statement. The second statement follows from Lemmas 2.4 and 2.5. Finally, the third and fourth statement follow directly from Lemma 2.3.

Corollary 2.8. The function

$$
\Psi_{a}(r, t):=\psi_{a}\left(\frac{r}{\sqrt{-2 t}}\right)
$$

satisfies

$$
\Psi_{a, t}>\Psi_{a} \Psi_{a, r r}-\frac{1}{2} \Psi_{a, r}^{2}+r^{-2}\left(1-\Psi_{a}\right)\left(r \Psi_{a, r}+2 \Psi_{a}\right)
$$

for $r \in\left[r_{*} a^{-1} \sqrt{-2 t},\left(1+\frac{1}{100} a^{-2}\right) \sqrt{-2 t}\right]$.

Proof. This follows immediately from Proposition 2.7.

In the remainder of this section, we will set up a barrier argument based on the functions $\psi_{a}$. We will assume throughout that $(M, g(t))$ is a 3 -dimensional ancient $\kappa$-solution which is non-compact, has positive sectional curvature, and is rotationally symmetric. After a reparametrization, the metric can be written in the form

$$
\tilde{g}(t)=u(r, t)^{-1} d r \otimes d r+r^{2} g_{S^{2}} .
$$

For each $t$, the function $r \mapsto u(r, t)$ is defined on an interval $\left[0, r_{\max }(t)\right)$, where $r_{\max }(t)$ may be finite or infinite.

The Ricci and scalar curvature of $\tilde{g}$ are given by

$$
\operatorname{Ric}_{\tilde{g}}=-\frac{1}{r} u^{-1} u_{r} d r \otimes d r+\left(1-u-\frac{1}{2} r u_{r}\right) g_{S^{2}}
$$

and

$$
R_{\tilde{g}}=\frac{2}{r^{2}}\left(1-u-r u_{r}\right)
$$

(cf. [5, p. 497]). Since the original metrics $g(t)$ evolve by the Ricci flow, the reparameterized metrics $\tilde{g}(t)$ satisfy an evolution equation of the form

$$
\frac{\partial}{\partial t} \tilde{g}=-2 \operatorname{Ric}_{\tilde{g}}+\mathscr{L}_{V}(\tilde{g})
$$

where $V$ is a radial vector field of the form $V=v(r, t) \partial / \partial r$, which may depend on time.

Clearly,

$$
\frac{\partial}{\partial t} \tilde{g}=-u^{-2} u_{t} d r \otimes d r .
$$


Moreover, $\mathscr{L}_{V}(r)=v$ and $\mathscr{L}_{V}(d r)=v_{r} d r$. This gives

$$
\mathscr{L}_{V}(\tilde{g})=\left(-u^{-2} u_{r} v+2 u^{-1} v_{r}\right) d r \otimes d r+2 r v g_{S^{2}},
$$

and hence

$$
\operatorname{Ric}_{\tilde{g}}-\frac{1}{2} \mathscr{L}_{V}(\tilde{g})=\left(-\frac{1}{r} u^{-1} u_{r}+\frac{1}{2} u^{-2} u_{r} v-u^{-1} v_{r}\right) d r \otimes d r+\left(1-u-\frac{1}{2} r u_{r}-r v\right) g_{S^{2}}
$$

Putting these facts together, we conclude that

$$
v=\frac{1}{r}\left(1-u-\frac{1}{2} r u_{r}\right)
$$

and

$$
u_{t}=2\left(-\frac{1}{r} u u_{r}+\frac{1}{2} u_{r} v-u v_{r}\right)=u u_{r r}-\frac{1}{2} u_{r}^{2}+r^{-2}(1-u)\left(r u_{r}+2 u\right) .
$$

The function $u$ has a natural geometric interpretation. Namely, we can view the radius $r$ as a scalar function on $M$. Then, $u=|d r|_{\tilde{g}(t)}^{2}$. In particular, $u$ is very small on a neck.

LEMma 2.9. We have $u(r, t) \leqslant 1, u_{r}(r, t) \leqslant 0$, and $v(r, t) \geqslant 0$ at each point in spacetime. Moreover, $1-u(r, t)=O\left(r^{2}\right)$ and $v(r, t)=O(r)$ as $r \rightarrow 0$.

Proof. As the metric is smooth at the tip, we obtain $1-u(r, t)=O\left(r^{2}\right)$ and $v(r, t)=$ $O(r)$ as $r \rightarrow 0$. Since $(M, g(t))$ has positive Ricci curvature, we have $u_{r}(r, t)=-r \operatorname{Ric}_{r}{ }^{r} \leqslant 0$ at each point in space-time. Integrating over $r$, we obtain $u(r, t) \leqslant 1$ at each point in space-time. Finally, $v(r, t)=\frac{1}{2} r\left(R-\mathrm{Ric}_{r}{ }^{r}\right) \geqslant 0$ at each point in space-time.

LEMMA 2.10. If a sphere of symmetry in $(M, g(t))$ has radius $r$, then its diameter in $(M, g(t))$ is at least $2 r$.

Proof. By Lemma 2.9, we have $u(r, t) \leqslant 1$. Consequently, the metric satisfies

$$
u(r, t)^{-1} d r \otimes d r+r^{2} g_{S^{2}} \geqslant d r \otimes d r+r^{2} g_{S^{2}} .
$$

This allows us to compare the distance function in $(M, g(t))$ to the distance function in Euclidean space. In particular, if we consider two antipodal points on a sphere of radius $r$, then their geodesic distance in $(M, g(t))$ is at least $2 r$.

LEMmA 2.11. Given any $\delta>0$, we have $\liminf _{t \rightarrow-\infty} \sup _{r \geqslant \delta \sqrt{-t}} u(r, t)=0$.

Proof. Let $\varepsilon>0$ be given. For each $t$, we denote by $R_{\max }(t)$ the supremum of the scalar curvature of $(M, g(t))$. By work of Perelman [21], the set of all points in $(M, g(t))$ which do not lie on an $\varepsilon$-neck has diameter less than $C(\varepsilon) R_{\max }(t)^{-1 / 2}$ (see Theorem A.2 
and Corollary A.3). Hence, if $r>C(\varepsilon) R_{\max }(t)^{-1 / 2}$ at some point in space-time, then that point lies on an $\varepsilon$-neck, and we have $u \leqslant 2 \varepsilon$. Thus,

$$
\sup _{r>C(\varepsilon) R_{\max }(t)^{-1 / 2}} u(r, t) \leqslant 2 \varepsilon
$$

for each $t$. On the other hand, we know that our ancient solution is of type II (cf. [25]), so that $\limsup _{t \rightarrow \infty}(-t) R_{\max }(t)=\infty$. Putting these facts together, we conclude that $\lim \inf _{t \rightarrow-\infty} \sup _{r \geqslant \delta \sqrt{-t}} u(r, t) \leqslant 2 \varepsilon$ for each $\delta>0$. Since $\varepsilon>0$ is arbitrary, it follows that $\lim \inf _{t \rightarrow-\infty} \sup _{r \geqslant \delta \sqrt{-t}} u(r, t)=0$ for each $\delta>0$. This completes the proof.

Proposition 2.12. There exists a large number $K$ with the following property. Suppose that $a \geqslant K$ and $\bar{t} \leqslant 0$. Moreover, suppose that $\bar{r}(t) \in\left[0, r_{\max }(t)\right)$ is a function satisfying

$$
\left|\frac{\bar{r}(t)}{\sqrt{-2 t}}-1\right| \leqslant \frac{1}{100} a^{-2} \quad \text { and } \quad u(\bar{r}(t), t)<\frac{1}{32} a^{-4}
$$

for all $t \leqslant \bar{t}$. Then $u(r, t) \leqslant \psi_{a}(r / \sqrt{-2 t})$ whenever $t \leqslant \bar{t}$ and $r_{*} a^{-1} \sqrt{-2 t} \leqslant r \leqslant \bar{r}(t)$. In particular, $u(r, t) \leqslant C a^{-2}$ whenever $t \leqslant \bar{t}$ and $\frac{1}{2} \sqrt{-2 t} \leqslant r \leqslant \bar{r}(t)$.

Proof. By Proposition 2.7, we can find a large constant $K$ such that $\psi_{a}(s) \geqslant \frac{1}{32} a^{-4}$ for all $s \in\left[r_{*} a^{-1}, 1+\frac{1}{100} a^{-2}\right]$ and all $a \geqslant K$. Moreover, we can arrange that $1+a^{-1} \beta_{a}\left(r_{*}\right)>0$ for all $a \geqslant K$.

We claim that $K$ has the desired property. To see this, we fix an arbitrary number $a \geqslant K$. Moreover, suppose that $\bar{r}(t) \in\left[0, r_{\max }(t)\right)$ is a function satisfying

$$
\left|\frac{\bar{r}(t)}{\sqrt{-2 t}}-1\right| \leqslant \frac{1}{100} a^{-2} \quad \text { and } \quad u(\bar{r}(t), t)<\frac{1}{32} a^{-4}
$$

for all $t \leqslant \bar{t}$. Then,

$$
\psi_{a}\left(\frac{\bar{r}(t)}{\sqrt{-2 t}}\right)-u(\bar{r}(t), t) \geqslant \frac{1}{32} a^{-4}-u(\bar{r}(t), t)>0
$$

for all $t \leqslant \bar{t}$. Moreover, since $\varphi\left(r_{*}\right)=2$ and $u \leqslant 1$, we have

$$
\psi_{a}\left(r_{*} a^{-1}\right)-u\left(r_{*} a^{-1} \sqrt{-2 t}, t\right)=2+a^{-1} \beta_{a}\left(r_{*}\right)-u\left(r_{*} a^{-1} \sqrt{-2 t}, t\right) \geqslant 1+a^{-1} \beta_{a}\left(r_{*}\right)>0
$$

for all $t \leqslant \bar{t}$. On the other hand, Lemma 2.11 implies that

$$
\limsup _{t \rightarrow-\infty} \inf _{r_{*} a^{-1} \sqrt{-2 t} \leqslant r \leqslant \bar{r}(t)}\left[\psi_{a}\left(\frac{r}{\sqrt{-2 t}}\right)-u(r, t)\right]>0 .
$$

Using Corollary 2.8 and the maximum principle, we obtain

$$
\psi_{a}\left(\frac{r}{\sqrt{-2 t}}\right)-u(r, t) \geqslant 0
$$

whenever $t \leqslant \bar{t}$ and $r_{*} a^{-1} \sqrt{-2 t} \leqslant r \leqslant \bar{r}(t)$. This completes the proof. 
Proposition 2.13. Suppose that there exists a function $\bar{r}(t) \in\left[0, r_{\max }(t)\right)$ such that $\bar{r}(t)=\sqrt{-2 t}+O(1)$ and $u(\bar{r}(t), t) \leqslant O(1 /-t)$ as $t \rightarrow-\infty$. Then, we can find a large constant $K \geqslant 100$ with the property that

$$
\frac{r}{\sqrt{-2 t+K a^{2}}} \leqslant 1+\frac{1}{100} a^{-2}
$$

and

$$
u(r, t) \leqslant \psi_{a}\left(\frac{r}{\sqrt{-2 t+K a^{2}}}\right)
$$

whenever $a \geqslant K, t \leqslant-K^{2} a^{2}$, and $r_{*} a^{-1} \sqrt{-2 t+K a^{2}} \leqslant r \leqslant \bar{r}(t)$. Note that $K$ is independent of $a$ and $t$. Moreover,

$$
\liminf _{t \rightarrow-\infty}(-t)^{-1} \int_{0}^{\bar{r}(t)} u(r, t)^{-1 / 2} d r>0 .
$$

Proof. We choose $K \geqslant 100$ sufficiently large so that the following holds:

- $\bar{r}(t) / \sqrt{-\left(2+K^{-1}\right) t} \geqslant 1-\theta$ for all $t \leqslant-K^{4}$;

- $\bar{r}(t)^{2}+2 t \leqslant \frac{1}{10} \sqrt{K} \bar{r}(t)$ for all $t \leqslant-K^{4}$;

- $u(\bar{r}(t), t) \leqslant K / 2 \bar{r}(t)^{2}$ for all $t \leqslant-K^{4}$;

- $\psi_{a}(s) \geqslant a^{-2}\left(s^{-2}-1\right)+\frac{1}{16} a^{-4}>0$ for all $s \in\left[1-\theta, 1+\frac{1}{100} a^{-2}\right]$ and all $a \geqslant K$;

- $1+a^{-1} \beta_{a}\left(r_{*}\right)>0$ for all $a \geqslant K$.

We claim that $K$ has the desired property. To prove this, we fix an arbitrary number $a \geqslant K$. Clearly,

$$
\frac{\bar{r}(t)}{\sqrt{-2 t+K a^{2}}} \geqslant \frac{\bar{r}(t)}{\sqrt{-\left(2+K^{-1}\right) t}} \geqslant 1-\theta
$$

for all $t \leqslant-K^{2} a^{2}$. Moreover, using the inequality $\bar{r}(t)^{2}+2 t \leqslant \frac{1}{10} \sqrt{K} \bar{r}(t)$, we obtain

$$
\begin{aligned}
\frac{-2 t+K a^{2}}{\bar{r}(t)^{2}}-1+\frac{1}{100} a^{-2} & =\frac{K a^{2}}{\bar{r}(t)^{2}}-\frac{\bar{r}(t)^{2}+2 t}{\bar{r}(t)^{2}}+\frac{1}{100} a^{-2} \\
& \geqslant \frac{K a^{2}}{\bar{r}(t)^{2}}-\frac{\sqrt{K}}{10 \bar{r}(t)}+\frac{1}{100} a^{-2} \\
& =\frac{3 K a^{2}}{4 \bar{r}(t)^{2}}+\left(\frac{\sqrt{K} a}{2 \bar{r}(t)}-\frac{1}{10} a^{-1}\right)^{2}
\end{aligned}
$$

for all $t \leqslant-K^{2} a^{2}$. Since the right-hand side is positive, it follows that

$$
\frac{\bar{r}(t)}{\sqrt{-2 t+K a^{2}}} \leqslant\left(1-\frac{1}{100} a^{-2}\right)^{-1 / 2} \leqslant 1+\frac{1}{100} a^{-2}
$$

for all $t \leqslant-K^{2} a^{2}$. This proves the first statement. 
We next observe that

$$
\begin{aligned}
\psi_{a}\left(\frac{\bar{r}(t)}{\sqrt{-2 t+K a^{2}}}\right)-u(\bar{r}(t), t) & \geqslant a^{-2}\left(\frac{-2 t+K a^{2}}{\bar{r}(t)^{2}}-1\right)+\frac{1}{100} a^{-4}-u(\bar{r}(t), t) \\
& \geqslant \frac{3 K}{4 \bar{r}(t)^{2}}-u(\bar{r}(t), t)>0
\end{aligned}
$$

for all $t \leqslant-K^{2} a^{2}$. Moreover, since $\varphi\left(r_{*}\right)=2$ and $u \leqslant 1$, we have

$$
\begin{aligned}
\psi_{a}\left(r_{*} a^{-1}\right)-u\left(r_{*} a^{-1} \sqrt{-2 t+K a^{2}}, t\right) & =2+a^{-1} \beta_{a}\left(r_{*}\right)-u\left(r_{*} a^{-1} \sqrt{-2 t+K a^{2}}, t\right) \\
& \geqslant 1+a^{-1} \beta_{a}\left(r_{*}\right)>0
\end{aligned}
$$

for all $t \leqslant-K^{2} a^{2}$. On the other hand, Lemma 2.11 implies

$$
\limsup _{t \rightarrow-\infty} \inf _{r_{*} a^{-1} \sqrt{-2 t+K a^{2}} \leqslant r \leqslant \bar{r}(t)}\left[\psi_{a}\left(\frac{r}{\sqrt{-2 t+K a^{2}}}\right)-u(r, t)\right]>0 .
$$

Using Corollary 2.8 and the maximum principle, we obtain

$$
\psi_{a}\left(\frac{r}{\sqrt{-2 t+K a^{2}}}\right)-u(r, t) \geqslant 0
$$

for all $t \leqslant-K^{2} a^{2}$ and $r_{*} a^{-1} \sqrt{-2 t+K a^{2}} \leqslant r \leqslant \bar{r}(t)$. This proves the second statement.

To prove the last statement, we recall that

$$
\frac{\bar{r}(t)}{\sqrt{-2 t+K a^{2}}} \geqslant 1-\theta
$$

for all $t \leqslant-K^{2} a^{2}$. Consequently,

$$
\begin{aligned}
\int_{r_{*} a^{-1} \sqrt{-2 t+K a^{2}}}^{\bar{r}(t)} u(r, t)^{-1 / 2} d r & \geqslant \int_{r_{*} a^{-1} \sqrt{-2 t+K a^{2}}}^{(1-\theta) \sqrt{-2 t+K a^{2}}} \psi_{a}\left(\frac{r}{\sqrt{-2 t+K a^{2}}}\right)^{-1 / 2} d r \\
& =\sqrt{-2 t+K a^{2}} \int_{r_{*} a^{-1}}^{1-\theta} \psi_{a}(s)^{-1 / 2} d s \\
& \geqslant \frac{a}{C} \sqrt{-2 t+K a^{2}}
\end{aligned}
$$

for $t \leqslant-K^{2} a^{2}$. To summarize, we have shown that

$$
\int_{0}^{\bar{r}(t)} u(r, t)^{-1 / 2} d r \geqslant \frac{a}{C} \sqrt{-t}
$$

whenever $a \geqslant K$ and $t \leqslant-K^{2} a^{2}$. Putting $t=-K^{2} a^{2}$, we conclude that

$$
\int_{0}^{\bar{r}(t)} u(r, t)^{-1 / 2} d r \geqslant \frac{1}{C K}(-t)
$$

for $t \leqslant-K^{4}$. 


\section{Asymptotics of ancient $\kappa$-solutions with rotational symmetry}

We continue to assume that $(M, g(t))$ is a 3 -dimensional ancient $\kappa$-solution which is noncompact, has positive sectional curvature, and is rotationally symmetric. Let $q \in M$ be a fixed reference point satisfying

$$
\sup _{t \leqslant 0}(-t) R(q, t) \leqslant 100
$$

such a point exists by Theorem A.4.

Proposition 3.1. If we dilate $(M, g(t))$ around $q$ by the factor $(-t)^{-1 / 2}$, then the rescaled manifolds converge in the Cheeger-Gromov sense to a cylinder of radius $\sqrt{2}$.

Proof. Recall that $\sup _{t \leqslant 0}(-t) R(q, t) \leqslant 100$ by our choice of $q$. Let $\ell$ denote the reduced distance from $(q, 0)$. Moreover, let us consider an arbitrary sequence of times $t_{k} \rightarrow-\infty$. Then,

$$
\ell\left(q, t_{k}\right) \leqslant \frac{1}{2 \sqrt{-t_{k}}} \int_{t_{k}}^{0} \sqrt{-t} R(q, t) d t \leqslant 1000
$$

if $k$ is sufficiently large. Let us dilate the flow $(M, g(t))$ around $\left(q, t_{k}\right)$ by the factor $\left(-t_{k}\right)^{-1 / 2}$. By work of Perelman, the rescaled flows converge in the Cheeger-Gromov sense to a shrinking gradient Ricci soliton (see [21, §11]), and this asymptotic soliton must be a cylinder (cf. $[22, \S 1])$.

For each $t$, we denote by $\bar{r}(t) \in\left[0, r_{\max }(t)\right)$ the radius of the sphere of symmetry passing through the point $q$. By Proposition 3.1, $\bar{r}(t) / \sqrt{-2 t} \rightarrow 1$ as $t \rightarrow-\infty$. Since $q$ is fixed, $\bar{r}(t)$ satisfies the following ODE:

$$
\frac{d}{d t} \bar{r}(t)=-v(\bar{r}(t), t)=-\frac{1}{\bar{r}(t)}\left(1-u(\bar{r}(t), t)-\frac{1}{2} \bar{r}(t) u_{r}(\bar{r}(t), t)\right) .
$$

We define a function $F(z, t)$ by

$$
F\left(\int_{\bar{r}(t)}^{\varrho} u(r, t)^{-1 / 2} d r, t\right)=\varrho .
$$

In other words, for each time $t$, the function $F(z, t)$ tells us the radius as a function of the signed distance $z$ from the reference point $q$. For each $t$, the function $z \mapsto F(z, t)$ is defined on the interval $[-d(t), \infty)$, where

$$
d(t)=\int_{0}^{\bar{r}(t)} u(r, t)^{-1 / 2} d r
$$

denotes the distance of the reference point $q$ from the tip. Note that $F(-d(t), t)=0$. 
Proposition 3.2. The function $F$ satisfies

$$
\begin{aligned}
0=F_{t}(z, t)-F_{z z}(z, t)+F(z, t)^{-1}\left(1+F_{z}(z, t)^{2}\right) \\
\quad+2 F_{z}(z, t)\left[-F(0, t)^{-1} F_{z}(0, t)+\int_{F(0, t)}^{F(z, t)} \frac{1}{r^{2}} u(r, t)^{1 / 2} d r\right] .
\end{aligned}
$$

Proof. Differentiating the identity

$$
\varrho=F\left(\int_{\bar{r}(t)}^{\varrho} u(r, t)^{-1 / 2} d r, t\right)
$$

with respect to $\varrho$ gives

$$
1=F_{z}\left(\int_{\bar{r}(t)}^{\varrho} u(r, t)^{-1 / 2} d r, t\right) u(\varrho, t)^{-1 / 2} .
$$

Taking another derivative with respect to $\varrho$ gives

$$
0=F_{z z}\left(\int_{\bar{r}(t)}^{\varrho} u(r, t)^{-1 / 2} d r, t\right) u(\varrho, t)^{-1}-\frac{1}{2} F_{z}\left(\int_{\bar{r}(t)}^{\varrho} u(r, t)^{-1 / 2} d r, t\right) u(\varrho, t)^{-3 / 2} u_{r}(\varrho, t) .
$$

Therefore,

$$
F_{z}\left(\int_{\bar{r}(t)}^{\varrho} u(r, t)^{-1 / 2} d r, t\right)=u(\varrho, t)^{1 / 2}
$$

and

$$
F_{z z}\left(\int_{\bar{r}(t)}^{\varrho} u(r, t)^{-1 / 2} d r, t\right)=\frac{1}{2} u_{r}(\varrho, t) .
$$

Using the identity

$$
\begin{aligned}
\frac{\partial}{\partial t}\left(u^{-1 / 2}\right) & =-\frac{1}{2} u^{-3 / 2}\left(u u_{r r}-\frac{1}{2} u_{r}^{2}+\frac{1}{r^{2}}(1-u)\left(r u_{r}+2 u\right)\right) \\
& =\frac{\partial}{\partial r}\left(\frac{1}{r} u^{-1 / 2}\left(1+u-\frac{1}{2} r u_{r}\right)\right)+\frac{2}{r^{2}} u^{1 / 2},
\end{aligned}
$$

we obtain

$$
\begin{aligned}
\frac{\partial}{\partial t}\left(\int_{\bar{r}(t)}^{\varrho} u(r, t)^{-1 / 2} d r\right)= & \frac{1}{\varrho} u(\varrho, t)^{-1 / 2}\left(1+u(\varrho, t)-\frac{1}{2} \varrho u_{r}(\varrho, t)\right) \\
& -\frac{1}{\bar{r}(t)} u(\bar{r}(t), t)^{-1 / 2}\left(1+u(\bar{r}(t), t)-\frac{1}{2} \bar{r}(t) u_{r}(\bar{r}(t), t)\right) \\
& \quad-u(\bar{r}(t), t)^{-1 / 2} \frac{d}{d t} \bar{r}(t)+\int_{\bar{r}(t)}^{\varrho} \frac{2}{r^{2}} u(r, t)^{1 / 2} d r \\
= & \frac{1}{\varrho} u(\varrho, t)^{-1 / 2}\left(1+u(\varrho, t)-\frac{1}{2} \varrho u_{r}(\varrho, t)\right) \\
& -\frac{2}{\bar{r}(t)} u(\bar{r}(t), t)^{1 / 2}+\int_{\bar{r}(t)}^{\varrho} \frac{2}{r^{2}} u(r, t)^{1 / 2} d r .
\end{aligned}
$$


Hence, if we differentiate the identity

$$
\varrho=F\left(\int_{\bar{r}(t)}^{\varrho} u(r, t)^{-1 / 2} d r, t\right)
$$

with respect to $t$, we find

$$
\begin{aligned}
0=F_{t} & \left(\int_{\bar{r}(t)}^{\varrho} u(r, t)^{-1 / 2} d r, t\right) \\
& +F_{z}\left(\int_{\bar{r}(t)}^{\varrho} u(r, t)^{-1 / 2} d r, t\right) \frac{1}{\varrho} u(\varrho, t)^{-1 / 2}\left(1+u(\varrho, t)-\frac{1}{2} \varrho u_{r}(\varrho, t)\right) \\
& +2 F_{z}\left(\int_{\bar{r}(t)}^{\varrho} u(r, t)^{-1 / 2} d r, t\right)\left(-\frac{1}{\bar{r}(t)} u(\bar{r}(t), t)^{1 / 2}+\int_{\bar{r}(t)}^{\varrho} \frac{1}{r^{2}} u(r, t)^{1 / 2} d r\right) .
\end{aligned}
$$

Putting these facts together, we conclude that

$$
\begin{aligned}
0=F_{t} & \left(\int_{\bar{r}(t)}^{\varrho} u(r, t)^{-1 / 2} d r, t\right)-F_{z z}\left(\int_{\bar{r}(t)}^{\varrho} u(r, t)^{-1 / 2} d r, t\right) \\
& +F\left(\int_{\bar{r}(t)}^{\varrho} u(r, t)^{-1 / 2} d r, t\right)^{-1}\left(1+F_{z}\left(\int_{\bar{r}(t)}^{\varrho} u(r, t)^{-1 / 2} d r, t\right)^{2}\right) \\
& +2 F_{z}\left(\int_{\bar{r}(t)}^{\varrho} u(r, t)^{-1 / 2} d r, t\right)\left(-\frac{1}{\bar{r}(t)} u(\bar{r}(t), t)^{1 / 2}+\int_{\bar{r}(t)}^{\varrho} \frac{1}{r^{2}} u(r, t)^{1 / 2} d r\right) .
\end{aligned}
$$

Using the relations $F(0, t)=\bar{r}(t)$ and $F_{z}(0, t)=u(\bar{r}(t), t)^{1 / 2}$, the assertion follows.

Corollary 3.3. The function F satisfies

$$
\begin{aligned}
& \left|F_{t}(z, t)-F_{z z}(z, t)+F(z, t)^{-1}\left(1+F_{z}(z, t)^{2}\right)\right| \\
& \leqslant 2 F(0, t)^{-1} F_{z}(0, t) F_{z}(z, t) \\
& \quad+2\left|\frac{1}{F(z, t)}-\frac{1}{F(0, t)}\right| \max \left\{F_{z}(z, t), F_{z}(0, t)\right\} F_{z}(z, t) .
\end{aligned}
$$

Proof. By Lemma 2.9, the function $u(r, t)$ is monotone decreasing in $r$. Hence, if $r$ lies in between $F(0, t)$ and $F(z, t)$, then $u(r, t)^{1 / 2} \leqslant \max \left\{F_{z}(z, t), F_{z}(0, t)\right\}$. This implies

$$
\left|\int_{F(0, t)}^{F(z, t)} \frac{1}{r^{2}} u(r, t)^{1 / 2} d r\right| \leqslant\left|\frac{1}{F(z, t)}-\frac{1}{F(0, t)}\right| \max \left\{F_{z}(z, t), F_{z}(0, t)\right\} .
$$

Therefore, the assertion follows from Proposition 3.2.

Proposition 3.4. We have the pointwise estimate

$$
F(z, t)^{m}\left|\partial_{z}^{m+1} F(z, t)\right| \leqslant C(m)\left(1+F(z, t)\left|F_{z z}(z, t)\right|\right)^{m}
$$

for each $m \geqslant 0$. 
Proof. We argue by induction on $m$. Lemma 2.9 implies $0 \leqslant F_{z} \leqslant 1$ at each point in space-time. Consequently, the assertion holds for $m=0$. Moreover, the assertion clearly holds for $m=1$.

Suppose now that $m \geqslant 2$, and the assertion holds for all integers less than $m$. Using the standard formula for the scalar curvature of a warped product, we obtain

$$
R=2 F^{-2}\left(1-F_{z}^{2}-2 F F_{z z}\right)
$$

Differentiating this identity with respect to $z$ gives

$$
\partial_{z}^{m-1} R+4 F^{-1} \partial_{z}^{m+1} F=\sum_{k=1}^{m+1} \sum_{\substack{i_{1} \geqslant 0, \ldots, i_{k} \geqslant 0 \\ i_{1}+\ldots+i_{k} \leqslant m-1}} c_{i_{1} \ldots i_{k}} F^{i_{1}+\ldots+i_{k}-m-1} \partial_{z}^{i_{1}+1} F \ldots \partial_{z}^{i_{k}+1} F .
$$

Using the induction hypothesis, we obtain

$$
\left|\partial_{z}^{m-1} R+4 F^{-1} \partial_{z}^{m+1} F\right| \leqslant C(m) F^{-m-1}\left(1+F\left|F_{z z}\right|\right)^{m-1} .
$$

On the other hand, Perelman's pointwise curvature derivative estimate (cf. [21]) implies

$$
\left|\partial_{z}^{m-1} R\right| \leqslant C(m) R^{(m+1) / 2} \leqslant C(m) F^{-m-1}\left(1+F\left|F_{z z}\right|\right)^{(m+1) / 2} .
$$

Putting these facts together, we conclude that

$$
\left|F^{-1} \partial_{z}^{m+1} F\right| \leqslant C(m) F^{-m-1}\left(1+F\left|F_{z z}\right|\right)^{m} .
$$

We now perform a rescaling. For $\tau \leqslant 0$, we define

$$
G(\xi, \tau):=e^{\tau / 2} F\left(e^{-\tau / 2} \xi,-e^{-\tau}\right)-\sqrt{2}
$$

Since $u(r, t)>0$ and $u_{r}(r, t) \leqslant 0$, it follows that $G_{\xi}(\xi, \tau)>0$ and $G_{\xi \xi}(\xi, \tau) \leqslant 0$.

Proposition 3.5. As $\tau \rightarrow-\infty, G(\xi, \tau) \rightarrow 0$ in $C_{\mathrm{loc}}^{\infty}$.

Proof. This follows from Proposition 3.1.

Proposition 3.6. The function $G$ satisfies

$$
\begin{aligned}
& \left|G_{\tau}(\xi, \tau)-G_{\xi \xi}(\xi, \tau)+\frac{1}{2} \xi G_{\xi}(\xi, \tau)-\frac{1}{2}(\sqrt{2}+G(\xi, \tau))+(\sqrt{2}+G(\xi, \tau))^{-1}\left(1+G_{\xi}(\xi, \tau)^{2}\right)\right| \\
& \leqslant 2(\sqrt{2}+G(0, \tau))^{-1} G_{\xi}(0, \tau) G_{\xi}(\xi, \tau) \\
& \quad+2\left|\frac{1}{\sqrt{2}+G(\xi, \tau)}-\frac{1}{\sqrt{2}+G(0, \tau)}\right| \max \left\{G_{\xi}(\xi, \tau), G_{\xi}(0, \tau)\right\} G_{\xi}(\xi, \tau) .
\end{aligned}
$$


Proof. This follows immediately from Corollary 3.3.

For each $k$, we define

$$
\begin{aligned}
\delta_{k}: & =\sup _{\tau \leqslant-k}|G(0, \tau)|+G_{\xi}(0, \tau) \\
& =\sup _{t \leqslant-e^{k}}\left|\frac{F(0, t)}{\sqrt{-t}}-\sqrt{2}\right|+F_{z}(0, t) \\
& =\sup _{t \leqslant-e^{k}}\left|\frac{\bar{r}(t)}{\sqrt{-t}}-\sqrt{2}\right|+u(\bar{r}(t), t)^{1 / 2} .
\end{aligned}
$$

By definition, $\delta_{k}$ is a decreasing sequence of positive numbers. Moreover, $\delta_{k} \rightarrow 0$ by Proposition 3.5.

LEMma 3.7. We have $|G(\xi, \tau)|+\left|G_{\xi}(\xi, \tau)\right| \leqslant C \delta_{k}^{1 / 4}$ for $\tau \leqslant-k$ and $|\xi| \leqslant 2 \delta_{k}^{-1 / 100}$.

Proof. By definition of $\delta_{k}$, we have

$$
\left|\frac{\bar{r}(t)}{\sqrt{-2 t}}-1\right| \leqslant \delta_{k} \quad \text { and } \quad u(\bar{r}(t), t) \leqslant \delta_{k}^{2}
$$

for all $t \leqslant-e^{k}$. We now apply Proposition 2.12 with $\bar{t}=-e^{k}$ and $a=\frac{1}{10} \delta_{k}^{-1 / 2}$. Using Proposition 2.12, we conclude that $u(r, t) \leqslant C \delta_{k}$ for all $t \leqslant-e^{k}$ and all $\frac{1}{2} \sqrt{-2 t} \leqslant r \leqslant \bar{r}(t)$. This implies that $0 \leqslant G_{\xi}(\xi, \tau) \leqslant C \delta_{k}^{1 / 2}$ whenever $\tau \leqslant-k$ and $-1 / \sqrt{2} \leqslant G(\xi, \tau) \leqslant G(0, \tau)$. Since $|G(0, \tau)| \leqslant \delta_{k}$ for $\tau \leqslant-k$, we conclude that $|G(\xi, \tau)|+\left|G_{\xi}(\xi, \tau)\right| \leqslant C \delta_{k}^{1 / 4}$ for all $\tau \leqslant-k$ and $-2 \delta_{k}^{-1 / 100} \leqslant \xi \leqslant 0$.

On the other hand, using the inequality $G_{\xi \xi}(\xi, \tau) \leqslant 0$, we obtain

$$
0 \leqslant G_{\xi}(\xi, \tau) \leqslant G_{\xi}(0, \tau) \leqslant \delta_{k}
$$

for all $\tau \leqslant-k$ and $0 \leqslant \xi \leqslant 2 \delta_{k}^{-1 / 100}$. Since $|G(0, \tau)| \leqslant \delta_{k}$, it follows that

$$
|G(\xi, \tau)|+\left|G_{\xi}(\xi, \tau)\right| \leqslant C \delta_{k}^{1 / 4}
$$

for $\tau \leqslant-k$ and $0 \leqslant \xi \leqslant 2 \delta_{k}^{-1 / 100}$.

LEMMA 3.8. We have $\left|G_{\xi \xi}(\xi, \tau)\right| \leqslant C \delta_{k}^{1 / 8}$ for $\tau \leqslant-k$ and $|\xi| \leqslant \delta_{k}^{-1 / 100}$.

Proof. Applying Proposition 3.4 with $m=2$, we obtain

$$
\left|G_{\xi \xi \xi}(\xi, \tau)\right| \leqslant C\left(1+\left|G_{\xi \xi}(\xi, \tau)\right|\right)^{2}
$$

for $\tau \leqslant-k$ and $|\xi| \leqslant 2 \delta_{k}^{-1 / 100}$. Moreover, Lemma 3.7 implies that

$$
\inf _{\xi^{\prime} \in\left[\xi-\delta_{k}^{1 / 8}, \xi+\delta_{k}^{1 / 8}\right]}\left|G_{\xi \xi}\left(\xi^{\prime}, \tau\right)\right| \leqslant C \delta_{k}^{1 / 8}
$$

for $\tau \leqslant-k$ and $|\xi| \leqslant \delta_{k}^{-1 / 100}$. Putting these facts together, we conclude that

$$
\left|G_{\xi \xi}(\xi, \tau)\right| \leqslant C \delta_{k}^{1 / 8}
$$

for $\tau \leqslant-k$ and $|\xi| \leqslant \delta_{k}^{-1 / 100}$. 
Lemma 3.9. We have $\left|\partial_{\xi}^{m+1} G(\xi, \tau)\right| \leqslant C(m)$ for $\tau \leqslant-k$ and $|\xi| \leqslant \delta_{k}^{-1 / 100}$.

Proof. Using Proposition 3.4, we obtain

$$
\left|\partial_{\xi}^{m+1} G(\xi, \tau)\right| \leqslant C(m)\left(1+\left|G_{\xi \xi}(\xi, \tau)\right|\right)^{m}
$$

for $\tau \leqslant-k$ and $|\xi| \leqslant 2 \delta_{k}^{-1 / 100}$. Moreover, Lemma 3.8 implies $\left|G_{\xi \xi}(\xi, \tau)\right| \leqslant C \delta_{k}^{1 / 8}$ for $\tau \leqslant-k$ and $|\xi| \leqslant \delta_{k}^{-1 / 100}$. Putting these facts together, the assertion follows.

LEMMA 3.10. We have

$$
\left|G_{\xi}(0, \tau)\right|^{4} \leqslant C \delta_{k}^{1 / 100} \int_{\left\{|\xi| \leqslant \delta_{k}^{-1 / 100}\right\}} e^{-\xi^{2} / 4}|G(\xi, \tau)|^{2} d \xi
$$

and

$$
\begin{gathered}
\int_{\left\{|\xi| \leqslant \delta_{k}^{-1 / 100}\right\}} e^{-\xi^{2} / 4}\left|G_{\xi}(\xi, \tau)\right|^{4} d \xi \leqslant C \delta_{k}^{1 / 100} \int_{\left\{|\xi| \leqslant \delta_{k}^{-1 / 100}\right\}} e^{-\xi^{2} / 4}|G(\xi, \tau)|^{2} d \xi \\
+C \exp \left(-\frac{1}{8} \delta_{k}^{-1 / 50}\right)
\end{gathered}
$$

for $\tau \leqslant-k$.

Proof. Using Lemma 3.9 and standard interpolation inequalities, we obtain

$$
\begin{aligned}
\left|G_{\xi}(0, \tau)\right|^{4} & \leqslant C\left(\int_{\{|\xi| \leqslant 1\}}|G(\xi, \tau)|^{2} d \xi\right)^{3 / 2} \\
& \leqslant C \delta_{k}^{1 / 100} \int_{\left\{|\xi| \leqslant \delta_{k}^{-1 / 100}\right\}} e^{-\xi^{2} / 4}|G(\xi, \tau)|^{2} d \xi
\end{aligned}
$$

for $\tau \leqslant-k$, where in the last step we have used Lemma 3.7. This proves the first statement. To prove the second statement, we observe that

$$
\begin{aligned}
& \int_{\left\{|\xi| \leqslant \delta_{k}^{-1 / 100}\right\}} e^{-\xi^{2} / 4} G_{\xi}(\xi, \tau)^{4} d \xi \\
& +3 \int_{\left\{|\xi| \leqslant \delta_{k}^{-1 / 100}\right\}} e^{-\xi^{2} / 4} G_{\xi}(\xi, \tau)^{2} G(\xi, \tau) G_{\xi \xi}(\xi, \tau) d \xi \\
& -\frac{1}{2} \int_{\left\{|\xi| \leqslant \delta_{k}^{-1 / 100}\right\}} e^{-\xi^{2} / 4} \xi G_{\xi}(\xi, \tau)^{3} G(\xi, \tau) d \xi \\
& \quad=\int_{\left\{|\xi| \leqslant \delta_{k}^{-1 / 100}\right\}} \frac{\partial}{\partial \xi}\left(e^{-\xi^{2} / 4} G_{\xi}(\xi, \tau)^{3} G(\xi, \tau)\right) d \xi \\
& \leqslant C \exp \left(-\frac{1}{8} \delta_{k}^{-1 / 50}\right)
\end{aligned}
$$


for $\tau \leqslant-k$, where in the last step we have used Lemma 3.7. Using Lemma 3.8, we obtain

$$
\begin{aligned}
& -3 \int_{\left\{|\xi| \leqslant \delta_{k}^{-1 / 100}\right\}} e^{-\xi^{2} / 4} G_{\xi}(\xi, \tau)^{2} G(\xi, \tau) G_{\xi \xi}(\xi, \tau) d \xi \\
& \quad \leqslant C \delta_{k}^{1 / 100} \int_{\left\{|\xi| \leqslant \delta_{k}^{-1 / 100}\right\}} e^{-\xi^{2} / 4} G_{\xi}(\xi, \tau)^{2}|G(\xi, \tau)| d \xi
\end{aligned}
$$

for $\tau \leqslant-k$. Moreover, Lemma 3.7 implies

$$
\begin{aligned}
& \frac{1}{2} \int_{\left\{|\xi| \leqslant \delta_{k}^{-1 / 100}\right\}} e^{-\xi^{2} / 4} \xi G_{\xi}(\xi, \tau)^{3} G(\xi, \tau) d \xi \\
& \quad \leqslant C \delta_{k}^{1 / 100} \int_{\left\{|\xi| \leqslant \delta_{k}^{-1 / 100}\right\}} e^{-\xi^{2} / 4} G_{\xi}(\xi, \tau)^{2}|G(\xi, \tau)| d \xi
\end{aligned}
$$

for $\tau \leqslant-k$. Adding these inequalities gives

$$
\begin{aligned}
& \int_{\left\{|\xi| \leqslant \delta_{k}^{-1 / 100}\right\}} e^{-\xi^{2} / 4} G_{\xi}(\xi, \tau)^{4} d \xi \\
& \leqslant C \delta_{k}^{1 / 100} \int_{\left\{|\xi| \leqslant \delta_{k}^{-1 / 100}\right\}} e^{-\xi^{2} / 4} G_{\xi}(\xi, \tau)^{2}|G(\xi, \tau)| d \xi \\
& +C \exp \left(-\frac{1}{8} \delta_{k}^{-1 / 50}\right) \\
& \leqslant C \delta_{k}^{1 / 100} \int_{\left\{|\xi| \leqslant \delta_{k}^{-1 / 100}\right\}} e^{-\xi^{2} / 4}\left(G_{\xi}(\xi, \tau)^{4}+G(\xi, \tau)^{2}\right) d \xi \\
& +C \exp \left(-\frac{1}{8} \delta_{k}^{-1 / 50}\right)
\end{aligned}
$$

for $\tau \leqslant-k$. Rearranging terms, the assertion follows.

LEMMA 3.11. We have

$$
\begin{aligned}
& \int_{\left\{|\xi| \leqslant \delta_{k}^{-1 / 100}\right\}} e^{-\xi^{2} / 4}\left|G_{\tau}(\xi, \tau)-G_{\xi \xi}(\xi, \tau)+\frac{1}{2} \xi G_{\xi}(\xi, \tau)-G(\xi, \tau)\right|^{2} d \xi \\
& \leqslant C \delta_{k}^{1 / 100} \int_{\left\{|\xi| \leqslant \delta_{k}^{-1 / 100}\right\}} e^{-\xi^{2} / 4}|G(\xi, \tau)|^{2} d \xi+C \exp \left(-\frac{1}{8} \delta_{k}^{-1 / 50}\right)
\end{aligned}
$$

for $\tau \leqslant-k$.

Proof. Note that

$$
\left|G(\xi, \tau)-\frac{1}{2}(\sqrt{2}+G(\xi, \tau))+(\sqrt{2}+G(\xi, \tau))^{-1}\right| \leqslant C G(\xi, \tau)^{2}
$$

for $\tau \leqslant-k$ and $|\xi| \leqslant \delta_{k}^{-1 / 100}$. Using Proposition 3.6, we obtain the pointwise estimate

$$
\begin{aligned}
\left|G_{\tau}(\xi, \tau)-G_{\xi \xi}(\xi, \tau)+\frac{1}{2} \xi G_{\xi}(\xi, \tau)-G(\xi, \tau)\right| & \\
\leqslant & C G(\xi, \tau)^{2}+C G_{\xi}(\xi, \tau)^{2}+C G_{\xi}(0, \tau) G_{\xi}(\xi, \tau) \\
\leqslant & C \delta_{k}^{1 / 100} G(\xi, \tau)+C G_{\xi}(\xi, \tau)^{2}+C G_{\xi}(0, \tau)^{2}
\end{aligned}
$$

for $\tau \leqslant-k$ and $|\xi| \leqslant \delta_{k}^{-1 / 100}$. Hence, the assertion follows from Lemma 3.10. 
We now perform a spectral decomposition for the operator $G_{\xi \xi}-\frac{1}{2} \xi G_{\xi}+G$. This operator is symmetric with respect to the inner product $\|G\|_{\mathcal{H}}^{2}=\int_{\mathbb{R}} e^{-|\xi|^{2} / 4} G^{2} d \xi$. The eigenvalues of this operator are given by $1-\frac{1}{2} n$, where $n \geqslant 0$. Moreover, the associated eigenfunctions are given by $H_{n}\left(\frac{1}{2} \xi\right)$, where $H_{n}$ is the $n$-th Hermite polynomial. Let us write $\mathcal{H}=\mathcal{H}_{+} \oplus \mathcal{H}_{0} \oplus \mathcal{H}_{-}$, where the subspace $\mathcal{H}_{+}$is defined as the span of $H_{0}\left(\frac{1}{2} \xi\right)$ and $H_{1}\left(\frac{1}{2} \xi\right)$, the subspace $\mathcal{H}_{0}$ is defined as the span of $H_{2}\left(\frac{1}{2} \xi\right)$, and $\mathcal{H}_{-}$is the orthogonal complement of $\mathcal{H}_{+} \oplus \mathcal{H}_{0}$. Moreover, let $P_{+}, P_{0}$, and $P_{-}$denote the orthogonal projections associated with the direct sum

$$
\mathcal{H}=\mathcal{H}_{+} \oplus \mathcal{H}_{0} \oplus \mathcal{H}_{-} .
$$

The eigenvalues of the operator $-G_{\xi \xi}+\frac{1}{2} \xi G_{\xi}-G$ on $\mathcal{H}_{+}$are bounded from above by $-\frac{1}{2}$. Similarly, the eigenvalues of the operator $-G_{\xi \xi}+\frac{1}{2} \xi G_{\xi}-G$ on $\mathcal{H}_{-}$are bounded from below by $\frac{1}{2}$.

Let $\chi$ denote a smooth cut-off function satisfying $\chi(s)=1$ for $s \in\left[-\frac{1}{2}, \frac{1}{2}\right], \chi(s)=0$ for $s \in \mathbb{R} \backslash[-1,1]$, and $s \chi^{\prime}(s) \leqslant 0$ for all $s \in \mathbb{R}$. We define

$$
\begin{aligned}
\gamma_{j} & :=\sup _{\tau \in[-j-1,-j]} \int_{\mathbb{R}} e^{-\xi^{2} / 4}\left|G(\xi, \tau) \chi\left(\delta_{j}^{1 / 100} \xi\right)\right|^{2} d \xi, \\
\gamma_{j}^{+} & :=\sup _{\tau \in[-j-1,-j]} \int_{\mathbb{R}} e^{-\xi^{2} / 4}\left|P_{+}\left(G(\xi, \tau) \chi\left(\delta_{j}^{1 / 100} \xi\right)\right)\right|^{2} d \xi, \\
\gamma_{j}^{0} & :=\sup _{\tau \in[-j-1,-j]} \int_{\mathbb{R}} e^{-\xi^{2} / 4}\left|P_{0}\left(G(\xi, \tau) \chi\left(\delta_{j}^{1 / 100} \xi\right)\right)\right|^{2} d \xi, \\
\gamma_{j}^{-} & :=\sup _{\tau \in[-j-1,-j]} \int_{\mathbb{R}} e^{-\xi^{2} / 4}\left|P_{-}\left(G(\xi, \tau) \chi\left(\delta_{j}^{1 / 100} \xi\right)\right)\right|^{2} d \xi .
\end{aligned}
$$

Clearly, $\frac{1}{C} \gamma_{j} \leqslant \gamma_{j}^{+}+\gamma_{j}^{0}+\gamma_{j}^{-} \leqslant C \gamma_{j}$. Using Lemma 3.7, we obtain

$$
\gamma_{j} \leqslant C \sup _{\tau \in[-j-1,-j]} \sup _{|\xi| \leqslant \delta_{j}^{-1 / 100}}|G(\xi, \tau)|^{2} \leqslant C \delta_{j}^{1 / 4} .
$$

In particular, $\gamma_{j} \rightarrow 0$.

LEMma 3.12. We have

$$
\begin{aligned}
\gamma_{j+1}^{+} & \leqslant e^{-1} \gamma_{j}^{+}+C \delta_{j}^{1 / 200}\left(\gamma_{j}+\gamma_{j+1}\right)+C \exp \left(-\frac{1}{64} \delta_{j}^{-1 / 50}\right), \\
\left|\gamma_{j+1}^{0}-\gamma_{j}^{0}\right| & \leqslant C \delta_{j}^{1 / 200}\left(\gamma_{j}+\gamma_{j+1}\right)+C \exp \left(-\frac{1}{64} \delta_{j}^{-1 / 50}\right) \\
\gamma_{j+1}^{-} & \geqslant e \gamma_{j}^{-}-C \delta_{j}^{1 / 200}\left(\gamma_{j}+\gamma_{j+1}\right)-C \exp \left(-\frac{1}{64} \delta_{j}^{-1 / 50}\right) .
\end{aligned}
$$


Proof. Fix $j$, and define $\widehat{G}(\xi, \tau):=G(\xi, \tau) \chi\left(\delta_{j}^{1 / 100} \xi\right)$. Note that

$$
\int_{\mathbb{R}} e^{-\xi^{2} / 4}|\widehat{G}(\xi, \tau)|^{2} d \xi \leqslant \gamma_{j}+\gamma_{j+1}
$$

for $\tau \in[-j-2,-j]$. Using Lemmas 3.11 and 3.7, we obtain

$$
\begin{gathered}
\int_{\mathbb{R}} e^{-\xi^{2} / 4}\left|\widehat{G}_{\tau}(\xi, \tau)-\widehat{G}_{\xi \xi}(\xi, \tau)+\frac{1}{2} \xi \widehat{G}_{\xi}(\xi, \tau)-\widehat{G}(\xi, \tau)\right|^{2} d \xi \\
\leqslant C \delta_{j}^{1 / 100}\left(\gamma_{j}+\gamma_{j+1}\right)+C \exp \left(-\frac{1}{32} \delta_{j}^{-1 / 50}\right)
\end{gathered}
$$

for $\tau \in[-j-2,-j]$. Consequently,

$$
\begin{aligned}
\frac{d}{d \tau}\left(\int_{\mathbb{R}} e^{-\xi^{2} / 4}\left|P_{+} \widehat{G}(\xi, \tau)\right|^{2} d \xi\right) \geqslant & \int_{\mathbb{R}} e^{-\xi^{2} / 4}\left|P_{+} \widehat{G}(\xi, \tau)\right|^{2} d \xi \\
& -C \delta_{j}^{1 / 200}\left(\gamma_{j}+\gamma_{j+1}\right)-C \exp \left(-\frac{1}{64} \delta_{j}^{-1 / 50}\right), \\
\left|\frac{d}{d \tau}\left(\int_{\mathbb{R}} e^{-\xi^{2} / 4}\left|P_{0} \widehat{G}(\xi, \tau)\right|^{2} d \xi\right)\right| \leqslant & C \delta_{j}^{1 / 200}\left(\gamma_{j}+\gamma_{j+1}\right)+C \exp \left(-\frac{1}{64} \delta_{j}^{-1 / 50}\right), \\
\frac{d}{d \tau}\left(\int_{\mathbb{R}} e^{-\xi^{2} / 4}\left|P_{-} \widehat{G}(\xi, \tau)\right|^{2} d \xi\right) \leqslant- & \int_{\mathbb{R}} e^{-\xi^{2} / 4}\left|P_{-} \widehat{G}(\xi, \tau)\right|^{2} d \xi \\
& +C \delta_{j}^{1 / 200}\left(\gamma_{j}+\gamma_{j+1}\right)+C \exp \left(-\frac{1}{64} \delta_{j}^{-1 / 50}\right)
\end{aligned}
$$

for $\tau \in[-j-2,-j]$. Integrating these inequalities over the interval $[\tau-1, \tau]$ gives

$$
\begin{gathered}
\int_{\mathbb{R}} e^{-\xi^{2} / 4}\left|P_{+} \widehat{G}(\xi, \tau-1)\right|^{2} d \xi \leqslant e^{-1} \int_{\mathbb{R}} e^{-\xi^{2} / 4}\left|P_{+} \widehat{G}(\xi, \tau)\right|^{2} d \xi \\
+C \delta_{j}^{1 / 200}\left(\gamma_{j}+\gamma_{j+1}\right)+C \exp \left(-\frac{1}{64} \delta_{j}^{-1 / 50}\right), \\
\left.\left|\int_{\mathbb{R}} e^{-\xi^{2} / 4}\right| P_{0} \widehat{G}(\xi, \tau-1)\right|^{2} d \xi-\int_{\mathbb{R}} e^{-\xi^{2} / 4}\left|P_{0} \widehat{G}(\xi, \tau)\right|^{2} d \xi \mid \\
\leqslant C \delta_{j}^{1 / 200}\left(\gamma_{j}+\gamma_{j+1}\right)+C \exp \left(-\frac{1}{64} \delta_{j}^{-1 / 50}\right), \\
\int_{\mathbb{R}} e^{-\xi^{2} / 4}\left|P_{-} \widehat{G}(\xi, \tau-1)\right|^{2} d \xi \geqslant e \int_{\mathbb{R}} e^{-\xi^{2} / 4}\left|P_{-} \widehat{G}(\xi, \tau)\right|^{2} d \xi \\
-C \delta_{j}^{1 / 200}\left(\gamma_{j}+\gamma_{j+1}\right)-C \exp \left(-\frac{1}{64} \delta_{j}^{-1 / 50}\right)
\end{gathered}
$$

for $\tau \in[-j-1,-j]$. We now define $\widetilde{G}(\xi, \tau):=G(\xi, \tau) \chi\left(\delta_{j+1}^{1 / 100} \xi\right)$. Using Lemma 3.7, we obtain

$$
\begin{aligned}
\int_{\mathbb{R}} e^{-\xi^{2} / 4}|\widetilde{G}(\xi, \tau-1)-\widehat{G}(\xi, \tau-1)|^{2} d \xi \\
\quad \leqslant \int_{\left\{\delta_{j}^{-1 / 100} / 2 \leqslant|\xi| \leqslant \delta_{j+1}^{-1 / 100}\right\}} e^{-\xi^{2} / 4|G(\xi, \tau-1)|^{2} d \xi} \\
\quad \leqslant C \exp \left(-\frac{1}{32} \delta_{j}^{-1 / 50}\right)
\end{aligned}
$$


for $\tau \in[-j-1,-j]$. Putting these facts together, we conclude that

$$
\begin{gathered}
\int_{\mathbb{R}} e^{-\xi^{2} / 4}\left|P_{+} \widetilde{G}(\xi, \tau-1)\right|^{2} d \xi \leqslant e^{-1} \int_{\mathbb{R}} e^{-\xi^{2} / 4}\left|P_{+} \widehat{G}(\xi, \tau)\right|^{2} d \xi \\
+C \delta_{j}^{1 / 200}\left(\gamma_{j}+\gamma_{j+1}\right)+C \exp \left(-\frac{1}{64} \delta_{j}^{-1 / 50}\right), \\
\left.\left|\int_{\mathbb{R}} e^{-\xi^{2} / 4}\right| P_{0} \widetilde{G}(\xi, \tau-1)\right|^{2} d \xi-\int_{\mathbb{R}} e^{-\xi^{2} / 4}\left|P_{0} \widehat{G}(\xi, \tau)\right|^{2} d \xi \mid \\
\leqslant C \delta_{j}^{1 / 200}\left(\gamma_{j}+\gamma_{j+1}\right)+C \exp \left(-\frac{1}{64} \delta_{j}^{-1 / 50}\right), \\
\int_{\mathbb{R}} e^{-\xi^{2} / 4}\left|P_{-} \widetilde{G}(\xi, \tau-1)\right|^{2} d \xi \geqslant e \int_{\mathbb{R}} e^{-\xi^{2} / 4}\left|P_{-} \widehat{G}(\xi, \tau)\right|^{2} d \xi \\
-C \delta_{j}^{1 / 200}\left(\gamma_{j}+\gamma_{j+1}\right)-C \exp \left(-\frac{1}{64} \delta_{j}^{-1 / 50}\right)
\end{gathered}
$$

for $\tau \in[-j-1,-j]$. Taking the supremum over $\tau \in[-j-1,-j]$, the assertion follows.

We next define

$$
\Gamma_{k}:=\sup _{j \geqslant k} \gamma_{j}, \quad \Gamma_{k}^{+}:=\sup _{j \geqslant k} \gamma_{j}^{+}, \quad \Gamma_{k}^{0}:=\sup _{j \geqslant k} \gamma_{j}^{0}, \quad \text { and } \quad \Gamma_{k}^{-}:=\sup _{j \geqslant k} \gamma_{j}^{-} .
$$

Clearly, $\Gamma_{k} / C \leqslant \Gamma_{k}^{+}+\Gamma_{k}^{0}+\Gamma_{k}^{-} \leqslant C \Gamma_{k}$. The inequality $\gamma_{j} \leqslant C \delta_{j}^{1 / 4}$ gives $\Gamma_{k} \leqslant C \delta_{k}^{1 / 4}$. In particular, $\Gamma_{k} \rightarrow 0$. Using Lemma 3.12, we obtain

$$
\begin{aligned}
\Gamma_{k+1}^{+} & \leqslant e^{-1} \Gamma_{k}^{+}+C \delta_{k}^{1 / 200} \Gamma_{k}+C \exp \left(-\frac{1}{64} \delta_{k}^{-1 / 50}\right), \\
\left|\Gamma_{k+1}^{0}-\Gamma_{k}^{0}\right| & \leqslant C \delta_{k}^{1 / 200} \Gamma_{k}+C \exp \left(-\frac{1}{64} \delta_{k}^{-1 / 50}\right), \\
\Gamma_{k+1}^{-} & \geqslant e \Gamma_{k}^{-}-C \delta_{k}^{1 / 200} \Gamma_{k}-C \exp \left(-\frac{1}{64} \delta_{k}^{-1 / 50}\right) .
\end{aligned}
$$

On the other hand, it follows from Lemma 3.9 and standard interpolation inequalities that

$$
\sup _{\tau \in[-j-1,-j]}|G(0, \tau)|+G_{\xi}(0, \tau) \leqslant C \gamma_{j}^{1 / 4}
$$

hence

$$
\delta_{k}=\sup _{\tau \leqslant-k}|G(0, \tau)|+G_{\xi}(0, \tau) \leqslant C \Gamma_{k}^{1 / 4} .
$$

Consequently, $\exp \left(-\frac{1}{64} \delta_{k}^{-1 / 50}\right) \leqslant C \delta_{k}^{5} \leqslant C \delta_{k} \Gamma_{k}$. Putting these facts together, we conclude that

$$
\begin{aligned}
\Gamma_{k+1}^{+} & \leqslant e^{-1} \Gamma_{k}^{+}+C \delta_{k}^{1 / 200} \Gamma_{k}, \\
\left|\Gamma_{k+1}^{0}-\Gamma_{k}^{0}\right| & \leqslant C \delta_{k}^{1 / 200} \Gamma_{k}, \\
\Gamma_{k+1}^{-} & \geqslant e \Gamma_{k}^{-}-C \delta_{k}^{1 / 200} \Gamma_{k} .
\end{aligned}
$$

The following lemma is inspired by a lemma of Merle and Zaag (cf. [19, Lemma A.1]). 
LEMmA 3.13. We either have $\Gamma_{k}^{0}+\Gamma_{k}^{-} \leqslant o(1) \Gamma_{k}^{+}$, or $\Gamma_{k}^{+}+\Gamma_{k}^{-} \leqslant o(1) \Gamma_{k}^{0}$.

Proof. By definition, the sequence $\Gamma_{k}^{-}$is monotone decreasing. This implies that $\Gamma_{k}^{-} \geqslant \Gamma_{k+1}^{-} \geqslant e \Gamma_{k}^{-}-o(1) \Gamma_{k}$. Thus, $\Gamma_{k}^{-} \leqslant o(1) \Gamma_{k}$. This gives $\Gamma_{k}^{-} \leqslant o(1)\left(\Gamma_{k}^{+}+\Gamma_{k}^{0}\right)$.

Let $I$ denote the set of all positive real numbers $\alpha$ with the property that the set $\left\{k: \Gamma_{k}^{0}<\alpha \Gamma_{k}^{+}\right\}$is finite. Moreover, let $J$ denote the set of all positive real numbers $\alpha$ with the property that the set $\left\{k: \Gamma_{k}^{0} \geqslant \alpha \Gamma_{k}^{+}\right\}$is infinite. Clearly, $I \subset J$.

We claim that $e^{1 / 2} \alpha \in I$ whenever $\alpha \in J$. To see this, suppose that $\alpha \in J$. We can find a large integer $k_{0}$ (depending on $\alpha$ ) such that

$$
\Gamma_{k+1}^{+} \leqslant e^{-1} \Gamma_{k}^{+}+\frac{1}{2(1+\alpha)}\left(e^{-1 / 2}-e^{-1}\right)\left(\Gamma_{k}^{+}+\Gamma_{k}^{0}\right)
$$

and

$$
\left|\Gamma_{k+1}^{0}-\Gamma_{k}^{0}\right| \leqslant \frac{\alpha}{2(1+\alpha)}\left(1-e^{-1 / 2}\right)\left(\Gamma_{k}^{+}+\Gamma_{k}^{0}\right)
$$

for all $k \geqslant k_{0}$. This implies that

$$
\begin{aligned}
\Gamma_{k+1}^{0}-e^{1 / 2} \alpha \Gamma_{k+1}^{+} & \geqslant \Gamma_{k}^{0}-e^{-1 / 2} \alpha \Gamma_{k}^{+}-\frac{\alpha}{1+\alpha}\left(1-e^{-1 / 2}\right)\left(\Gamma_{k}^{+}+\Gamma_{k}^{0}\right) \\
& =\left(1-\frac{\alpha}{1+\alpha}\left(1-e^{-1 / 2}\right)\right)\left(\Gamma_{k}^{0}-\alpha \Gamma_{k}^{+}\right)
\end{aligned}
$$

for all $k \geqslant k_{0}$. Since $\alpha \in J$, the set $\left\{k: \Gamma_{k}^{0} \geqslant \alpha \Gamma_{k}^{+}\right\}$is infinite. Hence, we can find an integer $k_{1} \geqslant k_{0}$ such that $\Gamma_{k}^{0}-\alpha \Gamma_{k}^{+} \geqslant 0$ for $k=k_{1}$. Proceeding inductively, we obtain $\Gamma_{k+1}^{0}-e^{1 / 2} \alpha \Gamma_{k+1}^{+} \geqslant 0$ for all $k \geqslant k_{1}$. Consequently, the set $\left\{k: \Gamma_{k}^{0}<e^{1 / 2} \alpha \Gamma_{k}^{+}\right\}$is finite. Thus, $e^{1 / 2} \alpha \in I$. This proves the claim.

Therefore, we may conclude that either $J=\varnothing$ or $I=(0, \infty)$. If $I=(0, \infty)$, we obtain $\Gamma_{k}^{+} \leqslant o(1) \Gamma_{k}^{0}$, and hence $\Gamma_{k}^{+}+\Gamma_{k}^{-} \leqslant o(1) \Gamma_{k}^{0}$. On the other hand, if $J=\varnothing$, then $\Gamma_{k}^{0} \leqslant o(1) \Gamma_{k}^{+}$, and hence $\Gamma_{k}^{0}+\Gamma_{k}^{-} \leqslant o(1) \Gamma_{k}^{+}$.

In the next step, we show that the second possibility in Lemma 3.13 cannot occur.

LEMma 3.14. We have $\Gamma_{k}^{0}+\Gamma_{k}^{-} \leqslant o(1) \Gamma_{k}^{+}$.

Proof. Suppose that the assertion is false. Lemma 3.13 implies that $\Gamma_{k}^{+}+\Gamma_{k}^{-} \leqslant o(1) \Gamma_{k}^{0}$. For each $k$, we can find an integer $j_{k} \geqslant k$ and a time $\tau_{k} \in\left[-j_{k}-1,-j_{k}\right]$ such that

$$
\Gamma_{k}=\gamma_{j_{k}}=\int_{\mathbb{R}} e^{-\xi^{2} / 4}\left|G\left(\xi, \tau_{k}\right) \chi\left(\delta_{j_{k}}^{1 / 100} \xi\right)\right|^{2} d \xi
$$

Note that

$$
\int_{\mathbb{R}} e^{-\xi^{2} / 4}\left|P_{+}\left(G\left(\xi, \tau_{k}\right) \chi\left(\delta_{j_{k}}^{1 / 100} \xi\right)\right)\right|^{2} d \xi \leqslant \gamma_{j_{k}}^{+} \leqslant \Gamma_{k}^{+} \leqslant o(1) \Gamma_{k}
$$


and

$$
\int_{\mathbb{R}} e^{-\xi^{2} / 4}\left|P_{-}\left(G\left(\xi, \tau_{k}\right) \chi\left(\delta_{j_{k}}^{1 / 100} \xi\right)\right)\right|^{2} d \xi \leqslant \gamma_{j_{k}}^{-} \leqslant \Gamma_{k}^{-} \leqslant o(1) \Gamma_{k} .
$$

After passing to a subsequence, the functions $\xi \mapsto \Gamma_{k}^{-1 / 2} G\left(\xi, \tau_{k}\right) \chi\left(\delta_{j_{k}}^{1 / 100} \xi\right)$ converge, in $\mathcal{H}$, to a non-zero multiple of the function $\xi^{2}-2$. Since the function $\xi \mapsto G\left(\xi, \tau_{k}\right)$ is monotone increasing, we have

$$
\int_{-3}^{-1} G\left(\xi, \tau_{k}\right) d \xi \leqslant \int_{-1}^{1} G\left(\xi, \tau_{k}\right) d \xi \leqslant \int_{1}^{3} G\left(\xi, \tau_{k}\right) d \xi
$$

for each $k$. Passing to the limit as $k \rightarrow \infty$, we obtain either

$$
\int_{-3}^{-1}\left(\xi^{2}-2\right) d \xi \leqslant \int_{-1}^{1}\left(\xi^{2}-2\right) d \xi \leqslant \int_{1}^{3}\left(\xi^{2}-2\right) d \xi
$$

or

$$
\int_{-3}^{-1}\left(2-\xi^{2}\right) d \xi \leqslant \int_{-1}^{1}\left(2-\xi^{2}\right) d \xi \leqslant \int_{1}^{3}\left(2-\xi^{2}\right) d \xi
$$

In either case, we arrive at a contradiction.

Lemma 3.15. We have $\Gamma_{k} \leqslant O\left(e^{-k}\right)$.

Proof. Note that $\Gamma_{k}^{0}+\Gamma_{k}^{-} \leqslant o(1) \Gamma_{k}^{+}$by Lemma 3.14. This implies

$$
\Gamma_{k+1}^{+} \leqslant e^{-1} \Gamma_{k}^{+}+C \delta_{k}^{1 / 200} \Gamma_{k}^{+} \leqslant e^{-1 / 2} \Gamma_{k}^{+}
$$

if $k$ is sufficiently large. Iterating this estimate gives $\Gamma_{k}^{+} \leqslant O\left(e^{-k / 2}\right)$, hence $\Gamma_{k} \leqslant O\left(e^{-k / 2}\right)$. Using the estimate $\delta_{k} \leqslant C \Gamma_{k}^{1 / 4}$, we obtain $\delta_{k} \leqslant O\left(e^{-k / 8}\right)$. This gives

$$
\Gamma_{k+1}^{+} \leqslant e^{-1} \Gamma_{k}^{+}+C \delta_{k}^{1 / 200} \Gamma_{k}^{+} \leqslant e^{-1} \Gamma_{k}^{+}+e^{-k / 2000} \Gamma_{k}^{+}
$$

if $k$ is sufficiently large. Iterating this estimate, we conclude that $\Gamma_{k}^{+} \leqslant O\left(e^{-k}\right)$, and hence $\Gamma_{k} \leqslant O\left(e^{-k}\right)$.

LEmma 3.16. We have $|G(0, \tau)| \leqslant O\left(e^{-k / 2}\right)$ and $\left|G_{\xi}(0, \tau)\right| \leqslant O\left(e^{-k / 2}\right)$ for all $\tau \leqslant-k$.

Proof. Lemma 3.15 gives

$$
\int_{\{|\xi| \leqslant 2\}}|G(\xi, \tau)|^{2} d \xi \leqslant O\left(e^{-k}\right)
$$

for all $\tau \leqslant-k$. Using Lemma 3.9 and standard interpolation inequalities, we obtain

$$
\sup _{|\xi| \leqslant 1}|G(\xi, \tau)|+\left|G_{\xi}(\xi, \tau)\right| \leqslant O\left(e^{-k / 3}\right)
$$


for all $\tau \leqslant-k$. Hence, Proposition 3.6 implies

$$
\sup _{|\xi| \leqslant 1}\left|G_{\tau}(\xi, \tau)-G_{\xi \xi}(\xi, \tau)+\frac{1}{2} \xi G_{\xi}(\xi, \tau)-G(\xi, \tau)\right| \leqslant O\left(e^{-2 k / 3}\right)
$$

for all $\tau \leqslant-k$. Using standard interior estimates for linear parabolic equations, we conclude that

$$
|G(0, \tau)| \leqslant O\left(e^{-k / 2}\right) \quad \text { and } \quad\left|G_{\xi}(0, \tau)\right| \leqslant O\left(e^{-k / 2}\right)
$$

for all $\tau \leqslant-k$.

After these preparations, we now prove the main result of this section.

Proposition 3.17. The function $d(t)$ satisfies $\liminf _{t \rightarrow-\infty}(-t)^{-1} d(t)>0$. Moreover, $\lim \inf _{t \rightarrow-\infty} R_{\max }(t)>0$, where $R_{\max }(t)$ denotes the supremum of the scalar curvature of $(M, g(t))$.

Proof. By Lemma 3.16, we have $|G(0, \tau)| \leqslant O\left(e^{\tau / 2}\right)$ and $G_{\xi}(0, \tau) \leqslant O\left(e^{\tau / 2}\right)$. Changing variables gives $|F(0, t)-\sqrt{-2 t}| \leqslant O(1)$ and $F_{z}(0, t) \leqslant O(1 / \sqrt{-t})$. Since $F(0, t)=\bar{r}(t)$ and $F_{z}(0, t)=u(\bar{r}(t), t)^{1 / 2}$, we obtain $|\bar{r}(t)-\sqrt{-2 t}| \leqslant O(1)$ and $u(\bar{r}(t), t) \leqslant O(1 /(-t))$. Applying Proposition 2.13, we conclude that

$$
\liminf _{t \rightarrow-\infty}(-t)^{-1} d(t)=\liminf _{t \rightarrow-\infty}(-t)^{-1} \int_{0}^{\bar{r}(t)} u(r, t)^{-1 / 2} d r>0
$$

We next observe that $d(t)=d_{g(t)}(p, q)$, where $p$ denotes the tip and $q$ is a fixed reference point on the manifold. Using Lemma 8.3 (b) in [21], we can control how fast the geodesic distance of $p$ and $q$ can grow as we go backwards in time:

$$
-\frac{d}{d t} d_{g(t)}(p, q) \leqslant C R_{\max }(t)^{1 / 2}
$$

Since $\liminf \operatorname{in}_{t \rightarrow-\infty}(-t)^{-1} d_{g(t)}(p, q)>0$, it follows that $\lim \sup _{t \rightarrow-\infty} R_{\max }(t)>0$. Since the function $t \mapsto R_{\max }(t)$ is monotone increasing by Hamilton's Harnack inequality [16], we conclude that $\liminf \operatorname{in}_{t \rightarrow-\infty} R_{\max }(t)>0$.

\section{Uniqueness of ancient $\kappa$-solutions with rotational symmetry}

We continue to assume that $(M, g(t))$ is a 3 -dimensional ancient $\kappa$-solution which is non-compact, has positive sectional curvature, and is rotationally symmetric. 
Proposition 4.1. Let $p$ denote the tip. Then $\liminf _{t \rightarrow-\infty} R(p, t)>0$.

Proof. Since the traceless Ricci tensor vanishes at the tip, the tip cannot lie on a neck. Hence, it follows from work of Perelman [21] that $R_{\max }(t) \leqslant C R(p, t)$ for some uniform constant $C$ (see Corollary A.3 below). Using Proposition 3.17, we obtain

$$
\liminf _{t \rightarrow-\infty} R(p, t)>0
$$

Let $p$ denote the tip. By Hamilton's trace Harnack inequality [16], the function $t \mapsto R(p, t)$ is monotone increasing. Hence, the limit

$$
\mathcal{R}:=\lim _{t \rightarrow-\infty} R(p, t)
$$

exists. Moreover, $\mathcal{R}>0$ by Proposition 4.1 .

Proposition 4.2. If we dilate $(M, g(t))$ around the tip by the factor $\mathcal{R}^{1 / 2}$, then the rescaled manifolds converge to the Bryant soliton in the Cheeger-Gromov sense.

Proof. Let $p$ denote the tip, and let $t_{k}$ be a sequence of times such that $t_{k} \rightarrow-\infty$. Let us dilate the flow around the point $\left(p, t_{k}\right)$ by the factor $\mathcal{R}^{1 / 2}$. The rescaled flows have uniformly bounded curvature. Hence, the rescaled flows converge in the Cheeger-Gromov sense to an eternal solution which is rotationally symmetric. Moreover, on the limiting eternal solution, the scalar curvature at the tip is equal to 1 at all times. Therefore, the limiting solution attains equality in Hamilton's Harnack inequality [16]. Consequently, the limit must be a steady gradient Ricci soliton [15]. Therefore, the limit must be the Bryant soliton.

We will need the following basic fact about the Bryant soliton.

LEMMA 4.3. Consider the Bryant soliton, normalized so that the scalar curvature at the tip is equal to 1. Let $\gamma$ be a geodesic ray emanating from the tip of the Bryant soliton which is parameterized by arclength. Then,

$$
\int_{0}^{\infty} \operatorname{Ric}\left(\gamma^{\prime}(s), \gamma^{\prime}(s)\right) d s=1 .
$$

Proof. On the Bryant soliton, we may write Ric $=D^{2} f$. This implies that

$$
\frac{d}{d s}\left\langle\nabla f(\gamma(s)), \gamma^{\prime}(s)\right\rangle=\left(D^{2} f\right)\left(\gamma^{\prime}(s), \gamma^{\prime}(s)\right)=\operatorname{Ric}\left(\gamma^{\prime}(s), \gamma^{\prime}(s)\right)
$$

Clearly, $\nabla f=0$ at the tip. Moreover, the identity $R+|\nabla f|^{2}=1$ implies that $|\nabla f| \rightarrow 1$ at infinity. Consequently, $\left\langle\nabla f(\gamma(s)), \gamma^{\prime}(s)\right\rangle=|\nabla f(\gamma(s))| \rightarrow 1$ as $s \rightarrow \infty$. Thus,

$$
\int_{0}^{\infty} \operatorname{Ric}\left(\gamma^{\prime}(s), \gamma^{\prime}(s)\right) d s=1 \text {. }
$$


We now continue with the the analysis of our ancient solution. As in $\S 3$, we define

$$
d(t)=\int_{0}^{\bar{r}(t)} u(r, t)^{-1 / 2} d r
$$

Equivalently, we may write $d(t)=d_{g(t)}(p, q)$, where $p$ denotes the tip and $q$ denotes the reference point introduced in $\S 3$. Clearly, $-d^{\prime}(t)>0$.

LEMmA 4.4. Let $\delta>0$ be given. Then,

$$
(1-\delta) \mathcal{R}^{1 / 2} \leqslant-d^{\prime}(t) \leqslant(1+\delta) \mathcal{R}^{1 / 2}
$$

if $-t$ is sufficiently large.

Proof. Let $p$ denote the tip, and let $\gamma$ denote the unit-speed geodesic in $(M, g(t))$ from the tip $p$ to our reference point $q$, so that $\gamma(0)=p$ and $\gamma(d(t))=q$. In view of Lemma 4.3 and Proposition 4.2, we can find a large constant $A$ (depending on $\delta$ ) such that $A \geqslant 8 \delta^{-1}$ and

$$
(1-\delta) \mathcal{R}^{1 / 2} \leqslant \int_{0}^{A \mathcal{R}^{-1 / 2}} \operatorname{Ric}_{g(t)}\left(\gamma^{\prime}(s), \gamma^{\prime}(s)\right) d s \leqslant\left(1+\frac{\delta}{2}\right) \mathcal{R}^{1 / 2}
$$

if $-t$ is sufficiently large (depending on $\delta$ and $A$ ).

We now observe that $\gamma$ is part of a minimizing geodesic ray emanating from the tip $p$. Hence, we may apply Theorem 17.4 (a) in [17] with $\sigma=A \mathcal{R}^{-1 / 2}$ and $L=d(t)+A \mathcal{R}^{-1 / 2}$. This gives

$$
0 \leqslant \int_{A \mathcal{R}^{-1 / 2}}^{d(t)} \operatorname{Ric}_{g(t)}\left(\gamma^{\prime}(s), \gamma^{\prime}(s)\right) d s \leqslant 4 A^{-1} \mathcal{R}^{1 / 2} .
$$

Putting these facts together, we obtain

$$
(1-\delta) \mathcal{R}^{1 / 2} \leqslant \int_{0}^{d(t)} \operatorname{Ric}_{g(t)}\left(\gamma^{\prime}(s), \gamma^{\prime}(s)\right) d s \leqslant\left(1+\frac{\delta}{2}+4 A^{-1}\right) \mathcal{R}^{1 / 2}
$$

if $-t$ is sufficiently large (depending on $\delta$ and $A$ ). Since

$$
d^{\prime}(t)=-\int_{0}^{d(t)} \operatorname{Ric}_{g(t)}\left(\gamma^{\prime}(s), \gamma^{\prime}(s)\right) d s
$$

it follows that

$$
(1-\delta) \mathcal{R}^{1 / 2} \leqslant-d^{\prime}(t) \leqslant\left(1+\frac{1}{2} \delta+4 A^{-1}\right) \mathcal{R}^{1 / 2},
$$

if $-t$ is sufficiently large (depending on $\delta$ and $A$ ). As $4 A^{-1} \leqslant \frac{1}{2} \delta$, the assertion follows. 
In the next step, we state a consequence of Hamilton's Harnack inequality. In the following, we view the scalar curvature $R$ as a function of $r$ and $t$. We denote by $R_{t}$ the partial derivative of $R$ with respect to $t$ (keeping $r$ fixed).

Proposition 4.5. We have

$$
R_{t}-\frac{2}{r} u^{-1} u_{t} v \geqslant 0
$$

Proof. Hamilton's trace Harnack inequality [16] implies that

$$
R_{t}-R_{r} v+2 R_{r} w+2 \mathrm{Ric}_{r r} w^{2} \geqslant 0,
$$

where

$$
v=\frac{1}{r}\left(1-u-\frac{1}{2} r u_{r}\right)
$$

and $w$ is arbitrary. The extra term $-R_{r} v$ arises because we compute the time derivative of the scalar curvature at a fixed radius $r$, whereas Hamilton computes the time derivative at a fixed point on the manifold. Indeed, if we fix a point on the manifold, then the radius $r$ shrinks at a rate given by $-v$, and the scalar curvature changes at a rate of $R_{t}-R_{r} v$.

Applying the Harnack inequality with $w:=v$ gives

$$
R_{t}+R_{r} v+2 \operatorname{Ric}_{r r} v^{2} \geqslant 0 .
$$

Note that

$$
R_{r}=-\frac{4}{r^{3}}\left(1-u+\frac{1}{2} r^{2} u_{r r}\right)=-\frac{2}{r} u^{-1} u_{t}+\frac{2}{r} u^{-1} u_{r} v
$$

and

$$
\mathrm{Ric}_{r r}=-\frac{1}{r} u^{-1} u_{r}
$$

and hence

$$
R_{r}+2 \operatorname{Ric}_{r r} v=-\frac{2}{r} u^{-1} u_{t} .
$$

Putting these facts together, the assertion follows.

We next consider the quantity $R+|V|^{2}=R+u^{-1} v^{2}$. Note that this function is smooth across the tip.

Remark 4.6. On the Bryant soliton, the function $u_{t}$ vanishes identically, and the function $R+u^{-1} v^{2}$ is equal to 1 .

Proposition 4.7. We have

$$
\left(R+u^{-1} v^{2}\right)_{t}+\frac{v}{2}\left(1+\frac{r}{2} u^{-1} v\right)^{-1}\left(R+u^{-1} v^{2}\right)_{r} \geqslant 0 .
$$


Proof. We observe that

$$
v=\frac{r}{4} R+\frac{1}{2 r}(1-u)
$$

hence

$$
v_{t}=\frac{r}{4} R_{t}-\frac{1}{2 r} u_{t}
$$

This gives

$$
\begin{aligned}
\left(R+u^{-1} v^{2}\right)_{t} & =R_{t}+2 u^{-1} v v_{t}-u^{-2} u_{t} v^{2} \\
& =\left(1+\frac{r}{2} u^{-1} v\right) R_{t}-\frac{1}{r} u^{-1} u_{t} v-u^{-2} u_{t} v^{2} \\
& =\left(1+\frac{r}{2} u^{-1} v\right)\left(R_{t}-\frac{2}{r} u^{-1} u_{t} v\right)+\frac{1}{r} u^{-1} u_{t} v
\end{aligned}
$$

Moreover, using the relations

$$
R_{r}=-\frac{2}{r} u^{-1} u_{t}+\frac{2}{r} u^{-1} u_{r} v
$$

and

we obtain

$$
u^{-2} u_{t}=-\frac{2}{r} u^{-1} u_{r}+u^{-2} u_{r} v-2 u^{-1} v_{r}
$$

$$
\begin{aligned}
\left(R+u^{-1} v^{2}\right)_{r} & =R_{r}-u^{-2} u_{r} v^{2}+2 u^{-1} v v_{r} \\
& =-\frac{2}{r} u^{-1} u_{t}+\frac{2}{r} u^{-1} u_{r} v-u^{-2} u_{r} v^{2}+2 u^{-1} v v_{r} \\
& =-\frac{2}{r} u^{-1} u_{t}-u^{-2} u_{t} v \\
& =-\frac{2}{r}\left(1+\frac{r}{2} u^{-1} v\right) u^{-1} u_{t} .
\end{aligned}
$$

Consequently,

$$
\left(R+u^{-1} v^{2}\right)_{t}+\frac{v}{2}\left(1+\frac{r}{2} u^{-1} v\right)^{-1}\left(R+u^{-1} v^{2}\right)_{r}=\left(1+\frac{r}{2} u^{-1} v\right)\left(R_{t}-\frac{2}{r} u^{-1} u_{t} v\right),
$$

and the right-hand side is non-negative by Proposition 4.5.

Proposition 4.8. The function $R+u^{-1} v^{2}$ satisfies

$$
\left(R+u^{-1} v^{2}\right)_{t}=u\left(R+u^{-1} v^{2}\right)_{r r}+\frac{2}{r} u\left(R+u^{-1} v^{2}\right)_{r}+\Xi(r, t)\left(R+u^{-1} v^{2}\right)_{r},
$$

where

$$
\Xi:=\left(1+u-\frac{1}{2} r u_{r}\right)^{-1}\left[\frac{1}{r}\left(1-\frac{1}{2} r u_{r}\right)\left(1-u-\frac{1}{2} r u_{r}\right)-u^{3} \partial_{r}\left(u^{-2}\left(1+u-\frac{1}{2} r u_{r}\right)\right)\right] .
$$

For each $t$, we have $\Xi(r, t)=O(r)$ near the tip. 
Proof. Differentiating the identity

$$
\left(R+u^{-1} v^{2}\right)_{r}=-\frac{2}{r}\left(1+\frac{r}{2} u^{-1} v\right) u^{-1} u_{t}=-\frac{1}{r} u^{-2}\left(1+u-\frac{1}{2} r u_{r}\right) u_{t}
$$

with respect to $r$ gives

$$
\begin{aligned}
\left(R+u^{-1} v^{2}\right)_{r r}=- & \frac{1}{r} u^{-2}\left(1+u-\frac{1}{2} r u_{r}\right) u_{t r}+\frac{1}{r^{2}} u^{-2}\left(1+u-\frac{1}{2} r u_{r}\right) u_{t} \\
& -\frac{1}{r} \partial_{r}\left(u^{-2}\left(1+u-\frac{1}{2} r u_{r}\right)\right) u_{t} .
\end{aligned}
$$

On the other hand, differentiating the identity

$$
R+u^{-1} v^{2}=\frac{1}{r^{2}} u^{-1}\left(1+u-\frac{1}{2} r u_{r}\right)^{2}-\frac{2}{r^{2}}(1+u)
$$

with respect to $t$ gives

$$
\begin{aligned}
\left(R+u^{-1} v^{2}\right)_{t}=- & \frac{1}{r} u^{-1}\left(1+u-\frac{1}{2} r u_{r}\right) u_{r t}-\frac{2}{r^{2}} u_{t} \\
& \quad-\frac{1}{r^{2}} u^{-2}\left(1+u-\frac{1}{2} r u_{r}\right)\left(1-u-\frac{1}{2} r u_{r}\right) u_{t} .
\end{aligned}
$$

Putting these facts together, we obtain

$$
\begin{aligned}
\left(R+u^{-1} v^{2}\right)_{t}-u\left(R+u^{-1} v^{2}\right)_{r r}-\frac{2}{r} u\left(R+u^{-1} v^{2}\right)_{r} \\
=-\left[\frac{1}{r}\left(1-\frac{1}{2} r u_{r}\right)\left(1-u-\frac{1}{2} r u_{r}\right)-u^{3} \partial_{r}\left(u^{-2}\left(1+u-\frac{1}{2} r u_{r}\right)\right)\right] \frac{1}{r} u^{-2} u_{t} \\
=\left[\frac{1}{r}\left(1-\frac{1}{2} r u_{r}\right)\left(1-u-\frac{1}{2} r u_{r}\right)-u^{3} \partial_{r}\left(u^{-2}\left(1+u-\frac{1}{2} r u_{r}\right)\right)\right] \\
\quad \times\left(1+u-\frac{1}{2} r u_{r}\right)^{-1}\left(R+u^{-1} v^{2}\right)_{r}
\end{aligned}
$$

as claimed.

Corollary 4.9. We have $R+u^{-1} v^{2} \geqslant \mathcal{R}$ at each point in space-time.

Proof. Let us fix a point $\left(r_{0}, t_{0}\right)$ in space-time such that $r_{0} \in\left[0, r_{\max }\left(t_{0}\right)\right)$. Let $\hat{r}(t)$ denote the solution of the ODE

$$
\frac{d}{d t} \hat{r}(t)=\frac{v(\hat{r}(t), t)}{2}\left(1+\frac{\hat{r}(t)}{2} u(\hat{r}(t), t)^{-1} v(\hat{r}(t), t)\right)^{-1}
$$

with initial condition $\hat{r}\left(t_{0}\right)=r_{0}$. Since $v$ is a non-negative function, we obtain $\hat{r}(t) \leqslant r_{0}$ for $t \leqslant t_{0}$. Consequently, the function $r \mapsto \hat{r}(t)$ is defined for all $t \in\left(-\infty, t_{0}\right]$, and $\hat{r}(t) \in$ $\left[0, r_{\max }(t)\right)$ for all $t \leqslant t_{0}$. 
By Proposition 4.7, the function

$$
t \longmapsto R(\hat{r}(t), t)+u(\hat{r}(t), t)^{-1} v(\hat{r}(t), t)^{2}
$$

is monotone increasing. On the other hand, by Proposition 4.2, we can find a sequence of times $t_{k} \rightarrow-\infty$ such that the rescaled manifolds $\left(M, \mathcal{R} g\left(t_{k}\right)\right)$ converge to the Bryant soliton in the Cheeger-Gromov sense. Since $R+u^{-1} v^{2}=1$ on the Bryant soliton, we conclude that

$$
\lim _{k \rightarrow \infty} \sup _{r \in\left(0, r_{0}\right]}\left|R\left(r, t_{k}\right)+u\left(r, t_{k}\right)^{-1} v\left(r, t_{k}\right)^{2}-\mathcal{R}\right|=0
$$

Consequently,

$$
\begin{aligned}
\mathcal{R} & =\lim _{k \rightarrow \infty} R\left(\hat{r}\left(t_{k}\right), t_{k}\right)+u\left(\hat{r}\left(t_{k}\right), t_{k}\right)^{-1} v\left(\hat{r}\left(t_{k}\right), t_{k}\right)^{2} \\
& \leqslant R\left(r_{0}, t_{0}\right)+u\left(r_{0}, t_{0}\right)^{-1} v\left(r_{0}, t_{0}\right)^{2} .
\end{aligned}
$$

Corollary 4.10. We have $\left(R+u^{-1} v^{2}\right)_{r} \geqslant 0$ at each point in space-time.

Proof. Let us fix a point $\left(r_{0}, t_{0}\right)$ in space-time such that $r_{0} \in\left[0, r_{\max }\left(t_{0}\right)\right)$. Let $\hat{r}(t)$ denote the solution of the ODE

$$
\frac{d}{d t} \hat{r}(t)=\frac{v(\hat{r}(t), t)}{2}\left(1+\frac{\hat{r}(t)}{2} u(\hat{r}(t), t)^{-1} v(\hat{r}(t), t)\right)^{-1}
$$

with initial condition $\hat{r}\left(t_{0}\right)=r_{0}$. Clearly, $\hat{r}(t) \leqslant r_{0}$ for $t \leqslant t_{0}$. Consequently, the function $r \mapsto \hat{r}(t)$ is defined for all $t \in\left(-\infty, t_{0}\right]$, and $\hat{r}(t) \in\left[0, r_{\max }(t)\right)$ for all $t \leqslant t_{0}$.

Let us consider an arbitrary sequence of times $t_{k} \rightarrow-\infty$. For $k$ large, we define

$$
Q_{k}=\left\{(r, t): t_{k} \leqslant t \leqslant t_{0}, r \leqslant \hat{r}(t)\right\} .
$$

By Proposition 4.8, the function $R+u^{-1} v^{2}$ attains its maximum on the parabolic boundary of $Q_{k}$. Therefore,

$$
\begin{aligned}
& \sup _{r \leqslant r_{0}} R\left(r, t_{0}\right)+u\left(r, t_{0}\right)^{-1} v\left(r, t_{0}\right)^{2} \\
& \quad \leqslant \max \left\{\sup _{t_{k} \leqslant t \leqslant t_{0}} R(\hat{r}(t), t)+u(\hat{r}(t), t)^{-1} v(\hat{r}(t), t)^{2}, \sup _{r \leqslant \hat{r}\left(t_{k}\right)} R\left(r, t_{k}\right)+u\left(r, t_{k}\right)^{-1} v\left(r, t_{k}\right)^{2}\right\}
\end{aligned}
$$

for $k$ large. By Proposition 4.7, the function

$$
t \longmapsto R(\hat{r}(t), t)+u(\hat{r}(t), t)^{-1} v(\hat{r}(t), t)^{2}
$$

is monotone increasing. This implies that

$$
\sup _{t_{k} \leqslant t \leqslant t_{0}} R(\hat{r}(t), t)+u(\hat{r}(t), t)^{-1} v(\hat{r}(t), t)^{2} \leqslant R\left(r_{0}, t_{0}\right)+u\left(r_{0}, t_{0}\right)^{-1} v\left(r_{0}, t_{0}\right)^{2}
$$


for $k$ large. This gives

$$
\begin{aligned}
\sup _{r \leqslant r_{0}} R\left(r, t_{0}\right)+u\left(r, t_{0}\right)^{-1} v\left(r, t_{0}\right)^{2} \\
\quad \leqslant \max \left\{R\left(r_{0}, t_{0}\right)+u\left(r_{0}, t_{0}\right)^{-1} v\left(r_{0}, t_{0}\right)^{2}, \sup _{r \leqslant \hat{r}\left(t_{k}\right)} R\left(r, t_{k}\right)+u\left(r, t_{k}\right)^{-1} v\left(r, t_{k}\right)^{2}\right\}
\end{aligned}
$$

for $k$ large. We now send $k \rightarrow \infty$. Recall that $\hat{r}\left(t_{k}\right) \leqslant r_{0}$ for $k$ large. Since the solution looks like the Bryant soliton near the tip, we obtain

$$
\lim _{k \rightarrow \infty} \sup _{r \leqslant \hat{r}\left(t_{k}\right)}\left|R\left(r, t_{k}\right)+u\left(r, t_{k}\right)^{-1} v\left(r, t_{k}\right)^{2}-\mathcal{R}\right|=0 .
$$

This gives

$$
\sup _{r \leqslant r_{0}} R\left(r, t_{0}\right)+u\left(r, t_{0}\right)^{-1} v\left(r, t_{0}\right)^{2} \leqslant \max \left\{R\left(r_{0}, t_{0}\right)+u\left(r_{0}, t_{0}\right)^{-1} v\left(r_{0}, t_{0}\right)^{2}, \mathcal{R}\right\} .
$$

Since $R\left(r_{0}, t_{0}\right)+u\left(r_{0}, t_{0}\right)^{-1} v\left(r_{0}, t_{0}\right)^{2} \geqslant \mathcal{R}$ by Corollary 4.9 , we conclude that

$$
\sup _{r \leqslant r_{0}} R\left(r, t_{0}\right)+u\left(r, t_{0}\right)^{-1} v\left(r, t_{0}\right)^{2} \leqslant R\left(r_{0}, t_{0}\right)+u\left(r_{0}, t_{0}\right)^{-1} v\left(r_{0}, t_{0}\right)^{2},
$$

which implies the claim.

LEMMA 4.11. Given $\varepsilon_{0}>0$, there is a large constant $C_{0}$ with the following property. If $r \geqslant C_{0}$ at some point in space-time, then that point lies at the center of an $\varepsilon_{0}$-neck.

Proof. By work of Perelman [21], the set of all points in $(M, g(t))$ which do not lie at the center of an $\varepsilon_{0}$-neck has diameter less than $C\left(\varepsilon_{0}\right) R_{\max }(t)^{-1 / 2}$ (see Theorem A.2 and Corollary A.3). Hence, if $r>C\left(\varepsilon_{0}\right) R_{\max }(t)^{-1 / 2}$ at some point in space-time, then that point lies at the center of an $\varepsilon_{0}$-neck. On the other hand, $R_{\max }(t)$ is uniformly bounded from below by Proposition 3.17. From this, the assertion follows.

Lemma 4.12. On an $\varepsilon_{0}$-neck, we have $r^{2} u \leqslant\left(1+100 \varepsilon_{0}\right) \mathcal{R}^{-1}$.

Proof. On an $\varepsilon_{0}$-neck, we have $u \leqslant \varepsilon_{0}$. Moreover, on an $\varepsilon_{0}$-neck, the radial Ricci curvature is smaller than $10 \varepsilon_{0} / r^{2}$. This gives $0 \leqslant-r u_{r} \leqslant 10 \varepsilon_{0}$. Using Corollary 4.9 , we obtain

$$
\mathcal{R} \leqslant R+u^{-1} v^{2}=\frac{1}{r^{2}} u^{-1}\left(1+u-\frac{1}{2} r u_{r}\right)^{2}-\frac{2}{r^{2}}(1+u) \leqslant \frac{1}{r^{2}} u^{-1}\left(1+100 \varepsilon_{0}\right) .
$$

This proves the assertion. 
LEMma 4.13. There is a large constant $C_{1}$ with the following property. If $F \geqslant C_{0}$, then we have $F\left|F_{z}\right| \leqslant C_{1}$ and $F^{2}\left|F_{z z}\right|+F^{3}\left|F_{z z z}\right| \leqslant C_{1} F^{1 / 100}$.

Proof. By Lemma 4.11, every point with $F \geqslant C_{0}$ lies at the center of an $\varepsilon_{0}$-neck. Using Lemma 4.12 , we obtain $F^{2} F_{z}^{2} \leqslant\left(1+100 \varepsilon_{0}\right) \mathcal{R}^{-1}$ on an $\varepsilon_{0}$-neck. We next observe that $F^{m}\left|\partial_{z}^{m+1} F\right| \leqslant C(m)$ on an $\varepsilon_{0}$-neck. Using standard interpolation inequalities, we obtain $F^{2}\left|F_{z z}\right|+F^{3}\left|F_{z z z}\right| \leqslant C F^{1 / 100}$ whenever $F \geqslant C_{0}$.

LEMma 4.14. There are large constants $C_{2} \geqslant 4 C_{0}$ and $C_{3}$ with the following property. If $F \geqslant C_{2}$, then $0 \leqslant-F_{z z} \leqslant C_{3} F^{-5 / 2+1 / 100}$.

Proof. Let us fix a point $\left(z_{0}, t_{0}\right)$ in space-time, and let $r_{0}=F\left(z_{0}, t_{0}\right) \in\left[0, r_{\max }\left(t_{0}\right)\right)$. We assume that $r_{0} \geqslant \max \left\{10 C_{0}, 100 C_{1}^{2}\right\}$. Let $\tilde{r}(t)$ denote the solution of the ODE

$$
\frac{d}{d t} \tilde{r}(t)=-v(\tilde{r}(t), t)=-\frac{1}{\tilde{r}(t)}\left(1-u(\tilde{r}(t), t)-\frac{1}{2} \tilde{r}(t) u_{r}(\tilde{r}(t), t)\right)
$$

with initial condition $\tilde{r}\left(t_{0}\right)=r_{0}$. Note that $\tilde{r}(t)$ can be interpreted as the radius, at time $t$, of a sphere of symmetry passing through a fixed point on the manifold. In particular, $\tilde{r}(t) \in\left[0, r_{\max }(t)\right)$ for $t \leqslant t_{0}$.

We define a function $\widetilde{F}(z, t)$ by

$$
\widetilde{F}\left(\int_{\tilde{r}(t)}^{\varrho} u(r, t)^{-1 / 2} d r, t\right)=\varrho .
$$

Clearly, $\widetilde{F}(0, t)=\tilde{r}(t)$. Note that $F(z, t)$ and $\widetilde{F}(z, t)$ differ only by a translation in $z$ :

$$
\widetilde{F}(z, t)=F\left(z+\int_{\bar{r}(t)}^{\tilde{r}(t)} u(r, t)^{-1 / 2} d r, t\right) .
$$

Since $\widetilde{F}\left(0, t_{0}\right)=F\left(z_{0}, t_{0}\right)=r_{0}$, we obtain $\widetilde{F}\left(z, t_{0}\right)=F\left(z+z_{0}, t_{0}\right)$ for all $z$.

Lemma 2.9 implies that $-d \tilde{r}(t) / d t \geqslant 0$ for each $t$. Integrating this inequality over $t$ gives $\tilde{r}(t) \geqslant r_{0}$ for all $t \leqslant t_{0}$. Equivalently, $\widetilde{F}(0, t) \geqslant r_{0}$ for all $t \leqslant t_{0}$. Moreover, Lemma 4.13 implies $\widetilde{F}\left|\widetilde{F}_{z}\right| \leqslant C_{1}$ whenever $\widetilde{F} \geqslant C_{0}$. Hence, if $r_{0} \geqslant \max \left\{10 C_{0}, 100 C_{1}^{2}\right\}$, then we obtain

$$
\widetilde{F}(z, t) \geqslant \sqrt{\widetilde{F}(0, t)^{2}-2 C_{1}|z|} \geqslant \sqrt{r_{0}^{2}-2 C_{1} r_{0}^{3 / 2}} \geqslant \frac{1}{2} r_{0}
$$

for all $t \leqslant t_{0}$ and all $z \in\left[-r_{0}^{3 / 2}, r_{0}^{3 / 2}\right]$.

The function $\widetilde{F}$ satisfies the evolution equation

$$
\begin{aligned}
& 0=\widetilde{F}_{t}(z, t)-\widetilde{F}_{z z}(z, t)+\widetilde{F}(z, t)^{-1}\left(1+\widetilde{F}_{z}(z, t)^{2}\right) \\
& \quad+2 \widetilde{F}_{z}(z, t)\left(-\widetilde{F}(0, t)^{-1} \widetilde{F}_{z}(0, t)+\int_{\widetilde{F}(0, t)}^{\widetilde{F}(z, t)} \frac{1}{r^{2}} u(r, t)^{1 / 2} d r\right) .
\end{aligned}
$$


This implies

$$
\begin{aligned}
0=\left(\widetilde{F}^{2}\right)_{t}(z, t)-\left(\widetilde{F}^{2}\right)_{z z}(z, t)+2+4 \widetilde{F}_{z}(z, t)^{2} & \\
& +4 \widetilde{F}(z, t) \widetilde{F}_{z}(z, t)\left(-\widetilde{F}(0, t)^{-1} \widetilde{F}_{z}(0, t)+\int_{\widetilde{F}(0, t)}^{\widetilde{F}(z, t)} \frac{1}{r^{2}} u(r, t)^{1 / 2} d r\right) .
\end{aligned}
$$

Consequently, if we define $\widetilde{Q}(z, t):=\frac{1}{2}\left(\widetilde{F}^{2}\right)_{z}(z, t)=\widetilde{F}(z, t) \widetilde{F}_{z}(z, t)$, then

$$
\begin{aligned}
0=\widetilde{Q}_{t}(z, t)-\widetilde{Q}_{z z}(z, t)+4 \widetilde{F}_{z}(z, t) \widetilde{F}_{z z}(z, t)+2 \widetilde{F}(z, t)^{-1} \widetilde{F}_{z}(z, t)^{3} \\
\quad+2\left(\widetilde{F}(z, t) \widetilde{F}_{z z}(z, t)+\widetilde{F}_{z}(z, t)^{2}\right)\left(-\widetilde{F}(0, t)^{-1} \widetilde{F}_{z}(0, t)+\int_{\widetilde{F}(0, t)}^{\widetilde{F}(t)} \frac{1}{r^{2}} u(r, t)^{1 / 2} d r\right) .
\end{aligned}
$$

Using Lemma 4.13, we obtain $|\widetilde{Q}(z, t)| \leqslant C$ and

$$
\left|\widetilde{Q}_{t}(z, t)-\widetilde{Q}_{z z}(z, t)\right| \leqslant C r_{0}^{-3+1 / 100}
$$

for $t \in\left[t_{0}-r_{0}^{3}, t_{0}\right]$ and $z \in\left[-r_{0}^{3 / 2}, r_{0}^{3 / 2}\right]$. Using standard interior estimates for linear parabolic equations, we conclude that $\left|\widetilde{Q}_{z}\left(0, t_{0}\right)\right| \leqslant C r_{0}^{-3 / 2+1 / 100}$. Consequently,

$$
\left|\widetilde{F}_{z z}\left(0, t_{0}\right)\right| \leqslant C r_{0}^{-5 / 2+1 / 100}
$$

This finally implies $\left|F_{z z}\left(z_{0}, t_{0}\right)\right| \leqslant C r_{0}^{-5 / 2+1 / 100}$.

LEMMA 4.15. There exist large constants $C_{4}$ and $C_{5}$ with the following property. If $-t \geqslant C_{4}$ and $F(z, t) \geqslant C_{4}$, then

$$
\left|F(z, t) F_{t}(z, t)+1\right| \leqslant C_{5} F(z, t)^{-3 / 2+1 / 100}+C_{5}(-t)^{-1}
$$

Proof. Recall that

$$
\begin{aligned}
& 0=F_{t}(z, t)-F_{z z}(z, t)+F(z, t)^{-1}\left(1+F_{z}(z, t)^{2}\right) \\
& \quad+2 F_{z}(z, t)\left(-F(0, t)^{-1} F_{z}(0, t)+\int_{F(0, t)}^{F(z, t)} \frac{1}{r^{2}} u(r, t)^{1 / 2} d r\right)
\end{aligned}
$$

Note that

$$
F(0, t)^{-1}\left|F_{z}(0, t)\right| \leqslant C(-t)^{-1}
$$

Moreover, if $F(z, t) \geqslant \max \left\{C_{0}, C_{2}\right\}$, then Lemmas 4.13 and 4.14 imply that

$$
\left|F_{z}(z, t)\right| \leqslant C F(z, t)^{-1} \quad \text { and } \quad\left|F_{z z}(z, t)\right| \leqslant C F(z, t)^{-5 / 2+1 / 100} .
$$

Putting these facts together, the assertion follows. 
LEMma 4.16. There is a large constant $C_{6}$ with the following property. If $-t \geqslant C_{6}$ and $F(z, t) \geqslant C_{6}$, then $-F(z, t) F_{t}(z, t) \geqslant \frac{1}{2}$.

Proof. This follows immediately from Lemma 4.15.

In the following, $p$ will denote the tip. By Proposition 4.2, we can find a large constant $C_{7}$ with the following property. If $-t \geqslant C_{7}$ and $x$ is a point in $(M, g(t))$ with $d_{g(t)}(p, x) \geqslant C_{7}$, then the sphere of symmetry passing through $x$ has radius

$$
r>\max \left\{C_{0}, C_{2}, C_{4}, C_{6}\right\}
$$

Moreover, let us fix a large constant $\Lambda \geqslant C_{7}$ such that

$$
d(t)=d_{g(t)}(p, q)<\Lambda
$$

for all $t \in\left[-\max \left\{C_{4}, C_{6}, C_{7}\right\}, 0\right]$.

For each $z \in(-\infty, 0]$, we define a time $\mathcal{T}(z) \in\left(-\infty,-\max \left\{C_{4}, C_{6}, C_{7}\right\}\right]$ by

$$
t=\mathcal{T}(z) \Longleftrightarrow d(t)=\Lambda-z .
$$

In other words, at time $\mathcal{T}(z)$, the reference point $q$ has distance $\Lambda-z$ from the tip.

Lemma 4.17. Let $z \leqslant 0$ and $t \leqslant \mathcal{T}(z)$. Then $F(z, t)>\max \left\{C_{0}, C_{2}, C_{4}, C_{6}\right\}$.

Proof. By assumption, $d(t)=d_{g(t)}(p, q) \geqslant \Lambda-z$. Let $x$ be a point in $(M, g(t))$ which has signed distance $z$ from the reference point $q$. Then $d_{g(t)}(p, x) \geqslant \Lambda$. In particular, $d_{g(t)}(p, x) \geqslant C_{7}$. Moreover, $-t \geqslant-\mathcal{T}(z) \geqslant C_{7}$. By our choice of $C_{7}$, the sphere of symmetry passing through $x$ has radius greater than $\max \left\{C_{0}, C_{2}, C_{4}, C_{6}\right\}$.

Lemma 4.18. Let $z \leqslant 0$ and $t=\mathcal{T}(z)$. Then, $F(z, t) \leqslant \Lambda$.

Proof. By assumption, $d(t)=d_{g(t)}(p, q)=\Lambda-z$. Let $x$ be a point in $(M, g(t))$ which has signed distance $z$ from the reference point $q$. Then $d_{g(t)}(p, x)=\Lambda$. Hence, the sphere of symmetry passing through $x$ has radius at most $\Lambda$.

LEMMA 4.19. There exists a large constant $C_{8}$ such that

$$
\left|F(z, t)^{2}-2(\mathcal{T}(z)-t)\right| \leqslant C_{8}(\mathcal{T}(z)-t)^{1 / 4+1 / 200}+C_{8}
$$

whenever $z \leqslant 0$ and $t \leqslant \mathcal{T}(z)$.

Proof. Using Lemmas 4.16 and 4.17 , we obtain $-F(z, t) F_{t}(z, t) \geqslant \frac{1}{2}$ whenever $z \leqslant 0$ and $t \leqslant \mathcal{T}(z)$. Integrating this inequality over $t$ gives $F(z, t)^{2} \geqslant \mathcal{T}(z)-t$ whenever $z \leqslant 0$ and $t \leqslant \mathcal{T}(z)$. 
Using Lemmas 4.15 and 4.17, we obtain

$$
\left|F(z, t) F_{t}(z, t)+1\right| \leqslant C F(z, t)^{-3 / 2+1 / 100}+C(-t)^{-1}
$$

whenever $z \leqslant 0$ and $t \leqslant \mathcal{T}(z)$. Using the inequality $F(z, t)^{2} \geqslant \mathcal{T}(z)-t$, we deduce that

$$
\left|F(z, t) F_{t}(z, t)+1\right| \leqslant C(\mathcal{T}(z)-t)^{-3 / 4+1 / 200}
$$

whenever $z \leqslant 0$ and $t \leqslant \mathcal{T}(z)$. Integrating this inequality over $t$ gives

$$
\left|F(z, t)^{2}-2(\mathcal{T}(z)-t)\right| \leqslant C(\mathcal{T}(z)-t)^{1 / 4+1 / 200}+C
$$

whenever $z \leqslant 0$ and $t \leqslant \mathcal{T}(z)$. In the last step, we have used the fact that, by Lemma 4.18, $F(z, t) \leqslant \Lambda$ whenever $z \leqslant 0$ and $t=\mathcal{T}(z)$.

Lemma 4.20. Let $\delta>0$ be given. Then, $F(0, t) F_{z}(0, t) \geqslant(1+4 \delta)^{-1} \mathcal{R}^{-1 / 2}$ if $-t$ is sufficiently large.

Proof. By Lemma 4.4,

$$
(1-\delta) \mathcal{R}^{1 / 2} \leqslant-d^{\prime}(t) \leqslant(1+\delta) \mathcal{R}^{1 / 2}
$$

if $-t$ is sufficiently large (depending on $\delta$ ). Integrating over $t$, we obtain

$$
(1-2 \delta) \mathcal{R}^{1 / 2}(-t) \leqslant d(t) \leqslant(1+2 \delta) \mathcal{R}^{1 / 2}(-t)
$$

if $-t$ is sufficiently large (depending on $\delta$ ). Putting $t=\mathcal{T}(z)$ gives

$$
(1+2 \delta)^{-1} \mathcal{R}^{-1 / 2}(\Lambda-z) \leqslant-\mathcal{T}(z) \leqslant(1-2 \delta)^{-1} \mathcal{R}^{-1 / 2}(\Lambda-z)
$$

if $-z$ is sufficiently large (depending on $\delta$ ).

In the following, we assume that $-t$ is sufficiently large, so that $t \leqslant \mathcal{T}(-\sqrt{-t}) \leqslant \mathcal{T}(0)$. We apply Lemma 4.19 with $z=0$ and, separately, with $z=-\sqrt{-t}$. This gives

$$
\left|F(0, t)^{2}-2(\mathcal{T}(0)-t)\right| \leqslant C_{8}(\mathcal{T}(0)-t)^{1 / 4+1 / 200}+C_{8}
$$

and

$$
\left|F(-\sqrt{-t}, t)^{2}-2(\mathcal{T}(-\sqrt{-t})-t)\right| \leqslant C_{8}(\mathcal{T}(-\sqrt{-t})-t)^{1 / 4+1 / 200}+C_{8} .
$$

This implies

$$
F(0, t)^{2}-F(-\sqrt{-t}, t)^{2}-2(\mathcal{T}(0)-\mathcal{T}(-\sqrt{-t})) \geqslant-2 C_{8}(-t)^{1 / 4+1 / 200}-2 C_{8} .
$$


Moreover,

$$
\mathcal{T}(0)-\mathcal{T}(-\sqrt{-t}) \geqslant(1+2 \delta)^{-1} \mathcal{R}^{-1 / 2}(\Lambda+\sqrt{-t})+\mathcal{T}(0)
$$

if $-t$ is sufficiently large (depending on $\delta$ ). Putting these facts together, we obtain

$$
F(0, t)^{2}-F(-\sqrt{-t}, t)^{2} \geqslant 2(1+2 \delta)^{-1} \mathcal{R}^{-1 / 2}(\Lambda+\sqrt{-t})+2 \mathcal{T}(0)-2 C_{8}(-t)^{1 / 4+1 / 200}-2 C_{8}
$$

if $-t$ is sufficiently large (depending on $\delta$ ). Consequently,

$$
\sup _{z \in[-\sqrt{-t}, 0]} F(z, t) F_{z}(z, t) \geqslant \frac{1}{2 \sqrt{-t}}\left(F(0, t)^{2}-F(-\sqrt{-t}, t)^{2}\right) \geqslant(1+3 \delta)^{-1} \mathcal{R}^{-1 / 2}
$$

if $-t$ is sufficiently large (depending on $\delta$ ). On the other hand, using Lemmas 4.13 and 4.14 , we obtain

$$
\left|\left(F F_{z}\right)_{z}\right|=\left|F F_{z z}+F_{z}^{2}\right| \leqslant C F^{-3 / 2+1 / 100} \leqslant C(-t)^{-3 / 4+1 / 200}
$$

for all $z \in[-\sqrt{-t}, 0]$. This implies

$$
\sup _{z \in[-\sqrt{-t}, 0]} F(z, t) F_{z}(z, t) \leqslant F(0, t) F_{z}(0, t)+C(-t)^{-1 / 4+1 / 200} .
$$

Thus, we conclude that

$$
F(0, t) F_{z}(0, t) \geqslant(1+4 \delta)^{-1} \mathcal{R}^{-1 / 2}
$$

if $-t$ is sufficiently large (depending on $\delta$ ).

The following lemma is similar to [9, Proposition 6.10].

Lemma 4.21. Let $\delta>0$ be given. Then,

$$
\inf _{z \geqslant 0} F(z, t) F_{z}(z, t) \geqslant(1+5 \delta)^{-1} \mathcal{R}^{-1 / 2}
$$

if $-t$ is sufficiently large.

Proof. Let us define

$$
Q(z, t):=\frac{1}{2}\left(F^{2}\right)_{z}(z, t)=F(z, t) F_{z}(z, t) .
$$

Note that $Q(z, t) \geqslant 0$. Moreover,

$$
\begin{aligned}
0=Q_{t} & (z, t)-Q_{z z}(z, t)+4 F_{z}(z, t) F_{z z}(z, t)+2 F(z, t)^{-1} F_{z}(z, t)^{3} \\
& +2\left(F(z, t) F_{z z}(z, t)+F_{z}(z, t)^{2}\right)\left(-F(0, t)^{-1} F_{z}(0, t)+\int_{F(0, t)}^{F(z, t)} \frac{1}{r^{2}} u(r, t)^{1 / 2} d r\right) .
\end{aligned}
$$


If $-t$ is sufficiently large, then $F(z, t) \geqslant F(0, t) \geqslant \sqrt{-t}$ for all $z \geqslant 0$. Using Lemma 4.13, we obtain

$$
\left|Q_{t}(z, t)-Q_{z z}(z, t)\right| \leqslant C F(z, t)^{-3+1 / 100}+C(-t)^{-1} F(z, t)^{-1+1 / 100} \leqslant C(-t)^{-3 / 2+1 / 200}
$$

for all $z \geqslant 0$. Let

$$
\widehat{Q}(z, t):=Q(z, t)+(-t)^{-1 / 2+1 / 100} .
$$

Clearly, $\inf _{z \geqslant 0} \widehat{Q}(z, t)>0$ for each $t$. Moreover, if $-t$ is sufficiently large, then

$$
\widehat{Q}_{t}(z, t)-\widehat{Q}_{z z}(z, t)>0
$$

for all $z \geqslant 0$. Finally, Lemma 4.20 implies that

$$
\widehat{Q}(0, t)>Q(0, t) \geqslant(1+4 \delta)^{-1} \mathcal{R}^{-1 / 2}
$$

if $-t$ is sufficiently large. Basic facts about the 1-dimensional heat equation on the half-line with Dirichlet boundary condition imply that

$$
\inf _{z \geqslant 0} \widehat{Q}(z, t) \geqslant(1+4 \delta)^{-1} \mathcal{R}^{-1 / 2}
$$

if $-t$ is sufficiently large. Consequently,

$$
\inf _{z \geqslant 0} Q(z, t) \geqslant(1+5 \delta)^{-1} \mathcal{R}^{-1 / 2}
$$

if $-t$ is sufficiently large.

LEMMA 4.22. If $-t$ is sufficiently large, then

$$
F(z, t)^{2} \geqslant \frac{1}{C_{9}}(z-t) \quad \text { and } \quad\left|\left(F F_{z}\right)_{t}\right| \leqslant C_{10} F^{-1+1 / 100}
$$

for all $z \geqslant 0$.

Proof. If $-t$ is sufficiently large, then Lemma 4.21 implies that

$$
F(z, t) F_{z}(z, t) \geqslant \frac{1}{C}
$$

for all $z \geqslant 0$. Integrating this inequality over $z$ gives

$$
F(z, t)^{2} \geqslant \frac{1}{C}(z-t)
$$

for all $z \geqslant 0$. This proves the first statement. To prove the second statement, we consider the function $Q(z, t):=F(z, t) F_{z}(z, t)$. We have shown above that

$$
\left|Q_{t}(z, t)-Q_{z z}(z, t)\right| \leqslant C F(z, t)^{-1+1 / 100}
$$

for all $z \geqslant 0$. Moreover, Lemma 4.13 implies that

$$
\left|Q_{z z}(z, t)\right|=\left|F(z, t) F_{z z z}(z, t)+F_{z}(z, t) F_{z z}(z, t)\right| \leqslant C F(z, t)^{-2+1 / 100}
$$

for all $z \geqslant 0$. Consequently, $\left|Q_{t}(z, t)\right| \leqslant C F(z, t)^{-1+1 / 100}$ for all $z \geqslant 0$. 
LEMMA 4.23. We have

$$
\lim _{z \rightarrow \infty} F(z, t) F_{z}(z, t)=\mathcal{R}^{-1 / 2}
$$

if $-t$ is sufficiently large.

Proof. Lemma 4.22 implies that $\liminf _{z \rightarrow \infty} F(z, t) F_{z}(z, t)$ is independent of $t$, provided that $-t$ is sufficiently large. On the other hand, it follows from Lemma 4.21 that

$$
\liminf _{z \rightarrow \infty} F(z, t) F_{z}(z, t) \geqslant(1+5 \delta)^{-1} \mathcal{R}^{-1 / 2}
$$

if $-t$ is sufficiently large (depending on $\delta$ ). Since $\delta$ is arbitrary, we conclude that

$$
\liminf _{z \rightarrow \infty} F(z, t) F_{z}(z, t) \geqslant \mathcal{R}^{-1 / 2}
$$

if $-t$ is sufficiently large. On the other hand, since $(M, g(t))$ is neck-like at spatial infinity, Lemma 4.12 implies that

$$
\limsup _{z \rightarrow \infty} F(z, t) F_{z}(z, t) \leqslant \mathcal{R}^{-1 / 2}
$$

for each $t$.

After these preparations, we now complete the proof of Theorem 1.1. Lemma 4.22 implies that $r_{\max }(t)=\infty$ if $-t$ is sufficiently large. By Lemma 4.23 , we have

$$
\lim _{z \rightarrow \infty} F(z, t) F_{z}(z, t)=\mathcal{R}^{-1 / 2}
$$

if $-t$ is sufficiently large. Equivalently, $\lim _{r \rightarrow \infty} r^{2} u(r, t)=\mathcal{R}^{-1}$ if $-t$ is sufficiently large. Moreover, since $(M, g(t))$ is neck-like at spatial infinity, we know that $\lim _{r \rightarrow \infty} u(r, t)=0$ and $\lim _{r \rightarrow \infty} r u_{r}(r, t)=0$. Using the identity

$$
R+u^{-1} v^{2}=\frac{1}{r^{2}} u^{-1}\left(1+u-\frac{1}{2} r u_{r}\right)^{2}-\frac{2}{r^{2}}(1+u),
$$

we obtain $\lim _{r \rightarrow \infty} R+u^{-1} v^{2}=\mathcal{R}$ if $-t$ is sufficiently large. Corollary 4.10 then implies $R+u^{-1} v^{2} \leqslant \mathcal{R}$ if $-t$ is sufficiently large. Using Corollary 4.9, we conclude $R+u^{-1} v^{2}=\mathcal{R}$ if $-t$ is sufficiently large. In view of the identity

$$
\left(R+u^{-1} v^{2}\right)_{r}=-\frac{2}{r}\left(1+\frac{r}{2} u^{-1} v\right) u^{-1} u_{t},
$$

it follows that $u_{t}=0$ if $-t$ is sufficiently large. Consequently, $(M, g(t))$ is a steady gradient Ricci soliton if $-t$ is sufficiently large. By the uniqueness result in [12], $(M, g(t))$ is a steady gradient Ricci soliton for all $t$. This completes the proof of Theorem 1.1. 


\section{Part II. Proof of Theorem 1.2}

\section{A PDE for the Lie derivative of the metric along a vector field}

We now study general solutions to the Ricci flow which are not necessarily rotationally

symmetric. Given a Riemannian metric $g$ and a symmetric $(0,2)$-tensor $h$, we define the Lichnerowicz Laplacian of $h$ by

$$
\Delta_{L, g} h_{i k}=\Delta h_{i k}+2 R_{i j k l} h^{j l}-\operatorname{Ric}_{i}^{l} h_{k l}-\operatorname{Ric}_{k}^{l} h_{i l} .
$$

Moreover, the divergence of $h$ is defined by

$$
(\operatorname{div} h)^{k}=D_{i} h^{i k}
$$

The following fact plays a key role in our analysis.

Proposition 5.1. Let $g$ be a Riemannian metric on a manifold $M$, and let $V$ be a vector field. We define $h:=\mathscr{L}_{V}(g)$ and $Z:=\operatorname{div} h-\frac{1}{2} \nabla(\operatorname{tr} h)$. Then,

$$
Z=\Delta V+\operatorname{Ric}(V)
$$

where Ric is viewed as a $(1,1)$-tensor. Moreover,

$$
\mathscr{L}_{V}(\mathrm{Ric})=-\frac{1}{2} \Delta_{L, g} h+\frac{1}{2} \mathscr{L}_{Z}(g),
$$

where Ric is viewed as a $(0,2)$-tensor.

Proof. Using the identity $h_{i j}=D_{i} V_{j}+D_{j} V_{i}$, we obtain

$$
Z_{k}=g^{i j} D_{i} h_{j k}-\frac{1}{2} g^{i j} D_{k} h_{i j}=g^{i j} D_{i, j}^{2} V_{k}+g^{i j} D_{i, k}^{2} V_{j}-g^{i j} D_{k, i}^{2} V_{j}=\Delta V_{k}+\operatorname{Ric}_{k}^{l} V_{l} .
$$

This proves the first statement.

To prove the second statement, let $\varphi_{s}: M \rightarrow M$ denote the 1-parameter family of diffeomorphisms generated by $V$. Then,

$$
\left.\frac{\partial}{\partial s} \varphi_{s}^{*}(g)\right|_{s=0}=h
$$

Using [23, Proposition 2.3.7], we obtain

$$
\mathscr{L}_{V}(\mathrm{Ric})=\left.\frac{\partial}{\partial s} \operatorname{Ric}_{\varphi_{s}^{*}(g)}\right|_{s=0}=-\frac{1}{2} \Delta_{L, g} h+\frac{1}{2} \mathscr{L}_{Z}(g) .
$$

We now state the main result of this section. 
COROLlary 5.2. Suppose that $g(t)$ is a solution to the Ricci flow on a manifold $M$. Moreover, suppose that $V(t)$ is a family of vector fields satisfying

$$
\frac{\partial}{\partial t} V(t)=\Delta_{g(t)} V(t)+\operatorname{Ric}_{g(t)}(V(t))
$$

Then, the Lie derivative $h(t):=\mathscr{L}_{V(t)}(g(t))$ satisfies the parabolic Lichnerowicz equation

$$
\frac{\partial}{\partial t} h(t)=\Delta_{L, g(t)} h(t)
$$

Proof. As above, let $Z:=\operatorname{div} h-\frac{1}{2} \nabla(\operatorname{tr} h)$. Proposition 5.1 implies that

$$
\frac{\partial}{\partial t} V=\Delta V+\operatorname{Ric}(V)=Z
$$

where Ric is viewed as a $(1,1)$-tensor. Moreover,

$$
\frac{\partial}{\partial t} g=-2 \text { Ric }
$$

where Ric is viewed as a $(0,2)$-tensor. Using Proposition 5.1 again, we obtain

$$
\frac{\partial}{\partial t} h=\mathscr{L}_{V}\left(\frac{\partial}{\partial t} g\right)+\mathscr{L}_{\partial V / \partial t}(g)=-2 \mathscr{L}_{V}(\text { Ric })+\mathscr{L}_{Z}(g)=\Delta_{L, g} h,
$$

where Ric is viewed as a $(0,2)$-tensor.

Proposition 5.3. Let $g(t)$ be a solution to the Ricci flow on a manifold $M$ and let $V(t)$ be a family of vector fields satisfying

$$
\frac{\partial}{\partial t} V(t)=\Delta_{g(t)} V(t)+\operatorname{Ric}_{g(t)}(V(t))+Q(t) .
$$

Then,

$$
\frac{\partial}{\partial t}|V(t)|_{g(t)} \leqslant \Delta_{g(t)}|V(t)|_{g(t)}+|Q(t)|_{g(t)}
$$

on the set $\{V(t) \neq 0\}$.

Proof. We compute

$$
\begin{aligned}
\frac{1}{2} \frac{\partial}{\partial t}\left(|V|^{2}\right) & =\left\langle V, \frac{\partial}{\partial t} V\right\rangle-\operatorname{Ric}(V, V)=\langle V, \Delta V\rangle+\langle V, Q\rangle \\
& =\frac{1}{2} \Delta\left(|V|^{2}\right)-|D V|^{2}+\langle V, Q\rangle \leqslant \frac{1}{2} \Delta\left(|V|^{2}\right)-|\nabla| V||^{2}+|V||Q|
\end{aligned}
$$

From this, the assertion easily follows. 


\section{The parabolic Lichnerowicz equation on shrinking cylinders}

In this section, we study the parabolic Lichnerowicz equation in a model case where the background metrics are a family of shrinking cylinders. Let $\left(S^{2} \times \mathbb{R}, \bar{g}(t)\right)$ be a family of shrinking cylinders evolving by Ricci flow, so that $\bar{g}(t)=(-2 t) g_{S^{2}}+d z \otimes d z$ for $t<0$.

Proposition 6.1. Let $h(t)$ be a 1-parameter family of symmetric $(0,2)$-tensors on the cylinder which is defined in the region $\left\{|z| \leqslant \frac{1}{2} L,-\frac{1}{2} L \leqslant t \leqslant-1\right\}$ and satisfies the parabolic Lichnerowicz equation

$$
\frac{\partial}{\partial t} h(t)=\Delta_{L, \bar{g}(t)} h(t)
$$

Assume that $|h(t)|_{\bar{g}(t)} \leqslant 1$ in the region $\left\{|z| \leqslant \frac{1}{2} L,-\frac{1}{2} L \leqslant t \leqslant-\frac{1}{4} L\right\}$, and $|h(t)|_{\bar{g}(t)} \leqslant L^{10}$ in the region $\left\{|z| \leqslant \frac{1}{2} L,-\frac{1}{4} L \leqslant t \leqslant-1\right\}$. On each slice $S^{2} \times\{z\}$, we may decompose the tensor $h(t)$ as

$$
h(t)=\omega(z, t) g_{S^{2}}+\chi(z, t)+d z \otimes \sigma(z, t)+\sigma(z, t) \otimes d z+\beta(z, t) d z \otimes d z,
$$

where $\omega(z, t)$ is a scalar function on $S^{2}, \chi(z, t)$ is a trace-free symmetric $(0,2)$-tensor on $S^{2}, \sigma(z, t)$ is a 1 -form on $S^{2}$, and $\beta(z, t)$ is a scalar function on $S^{2}$. Then, there exists a function $\psi: S^{2} \rightarrow \mathbb{R}$ (independent of $t$ and $z$ ) such that $\psi$ lies in the span of the first spherical harmonics on $S^{2}$, and

$$
\left|h(t)-\bar{\omega}(z, t) g_{S^{2}}-\bar{\beta}(z, t) d z \otimes d z-(-t) \psi g_{S^{2}}\right|_{\bar{g}(t)} \leqslant C L^{-1 / 2}
$$

in the region $\{|z| \leqslant 1000,-1000 \leqslant t \leqslant-1\}$. Here, $\bar{\omega}(z, t)$ and $\bar{\beta}(z, t)$ are rotationally invariant functions satisfying

$$
\int_{S^{2} \times\{z\}}(\omega(z, t)-\bar{\omega}(z, t)) d \mathrm{vol}_{S^{2}}=\int_{S^{2} \times\{z\}}(\beta(z, t)-\bar{\beta}(z, t)) d \mathrm{vol}_{S^{2}}=0
$$

for $t \in[-1000,-1]$ and $z \in[-1000,1000]$. In other words, $\bar{\omega}(z, t)$ and $\bar{\beta}(z, t)$ are obtained from $\omega(z, t)$ and $\beta(z, t)$ by averaging over the individual 2 -spheres $S^{2} \times\{z\}$.

Proof. The parabolic Lichnerowicz equation is equivalent to the following system of equations for $\omega(z, t), \chi(z, t), \sigma(z, t)$, and $\beta(z, t)$ :

$$
\begin{aligned}
& \frac{\partial}{\partial t} \omega(z, t)=\frac{\partial^{2}}{\partial z^{2}} \omega(z, t)+\frac{1}{-2 t} \Delta_{S^{2}} \omega(z, t) \\
& \frac{\partial}{\partial t} \chi(z, t)=\frac{\partial^{2}}{\partial z^{2}} \chi(z, t)+\frac{1}{-2 t}\left(\Delta_{S^{2}} \chi(z, t)-4 \chi(z, t)\right), \\
& \frac{\partial}{\partial t} \sigma(z, t)=\frac{\partial^{2}}{\partial z^{2}} \sigma(z, t)+\frac{1}{-2 t}\left(\Delta_{S^{2}} \sigma(z, t)-\sigma(z, t)\right), \\
& \frac{\partial}{\partial t} \beta(z, t)=\frac{\partial^{2}}{\partial z^{2}} \beta(z, t)+\frac{1}{-2 t} \Delta_{S^{2}} \beta(z, t) .
\end{aligned}
$$


By assumption, $|h(t)|_{\bar{g}(t)} \leqslant 1$ in the region $\left\{|z| \leqslant \frac{1}{2} L,-\frac{1}{2} L \leqslant t \leqslant-\frac{1}{4} L\right\}$, and $|h(t)|_{\bar{g}(t)} \leqslant L^{10}$ in the region $\left\{|z| \leqslant \frac{1}{2} L,-\frac{1}{4} L \leqslant t \leqslant-1\right\}$. This implies that

$$
\begin{aligned}
|\omega(z, t)| & \leqslant C(-t), \\
|\chi(z, t)|_{g_{S^{2}}} & \leqslant C(-t), \\
|\sigma(z, t)|_{S_{S^{2}}} & \leqslant C(-t)^{1 / 2}, \\
|\beta(z, t)| & \leqslant C
\end{aligned}
$$

in the region $\left\{|z| \leqslant \frac{1}{2} L,-\frac{1}{2} L \leqslant t \leqslant-\frac{1}{4} L\right\}$, and

$$
\begin{aligned}
|\omega(z, t)| & \leqslant C L^{10}(-t), \\
|\chi(z, t)|_{g_{S^{2}}} & \leqslant C L^{10}(-t), \\
|\sigma(z, t)|_{S_{S^{2}}} & \leqslant C L^{10}(-t)^{1 / 2}, \\
|\beta(z, t)| & \leqslant C L^{10}
\end{aligned}
$$

in the region $\left\{|z| \leqslant \frac{1}{2} L,-\frac{1}{4} L \leqslant t \leqslant-1\right\}$.

Step 1. We first analyze the equation for $\chi(z, t)$. Let $S_{j}, j=1,2, \ldots$, denote the eigenfunctions of the Laplacian on trace-free symmetric $(0,2)$-tensors on $S^{2}$, so that

$$
\Delta_{S^{2}} S_{j}=-\nu_{j} S_{j}
$$

Clearly, $\nu_{j}>0$ for each $j$. We assume that the eigenfunctions $S_{j}$ are normalized so that

$$
\int_{S^{2}}\left|S_{j}\right|_{g_{S^{2}}}^{2} d \mathrm{vol}_{S^{2}}=1
$$

for each $j$. Then, $\sup _{S^{2}}\left|S_{j}\right|_{g_{S^{2}}} \leqslant C\left\|S_{j}\right\|_{H^{2}} \leqslant C \nu_{j}$ for each $j$. Moreover, $\nu_{j} \sim j$ as $j \rightarrow \infty$ (cf. [7, Corollary 2.43]). Let us write

$$
\chi(z, t)=\sum_{j=1}^{\infty} \chi_{j}(z, t) S_{j},
$$

where

$$
\chi_{j}(z, t)=\int_{S^{2}}\left\langle\chi(z, t), S_{j}\right\rangle_{S_{S^{2}}} d \operatorname{vol}_{S^{2}} .
$$

Note that $\left|\chi_{j}(z, t)\right| \leqslant C \sup _{S^{2}}|\chi(z, t)|_{g^{2}}$. Moreover, the function $\chi_{j}(z, t)$ satisfies

$$
\frac{\partial}{\partial t} \chi_{j}(z, t)=\frac{\partial^{2}}{\partial z^{2}} \chi_{j}(z, t)-\frac{\nu_{j}+4}{-2 t} \chi_{j}(z, t) .
$$


Hence, the function $\widehat{\chi}_{j}(z, t):=(-t)^{-\left(\nu_{j}+4\right) / 2} \chi_{j}(z, t)$ satisfies

$$
\frac{\partial}{\partial t} \widehat{\chi}_{j}(z, t)=\frac{\partial^{2}}{\partial z^{2}} \widehat{\chi}_{j}(z, t)
$$

Moreover,

$$
\left|\widehat{\chi}_{j}(z, t)\right| \leqslant C(-t)^{-\left(\nu_{j}+2\right) / 2}
$$

in the region $\left\{|z| \leqslant \frac{1}{2} L,-\frac{1}{2} L \leqslant t \leqslant-\frac{1}{4} L\right\}$, and

$$
\left|\widehat{\chi}_{j}(z, t)\right| \leqslant C L^{20}(-t)^{-\left(\nu_{j}+2\right) / 2}
$$

in $\left\{|z| \leqslant \frac{1}{2} L,-\frac{1}{4} L \leqslant t \leqslant-1\right\}$. Using the solution formula for the Dirichlet problem for the 1 -dimensional heat equation on the rectangle $\left[-\frac{1}{4} L, \frac{1}{4} L\right] \times\left[-\frac{1}{4} L,-1\right]$, we obtain

$$
\begin{aligned}
\left|\widehat{\chi}_{j}(z, t)\right| \leqslant C & \sup _{z \in[-L / 4, L / 4]}\left|\widehat{\chi}_{j}\left(z,-\frac{1}{4} L\right)\right| \\
& +C L \int_{-L / 4}^{t} e^{-L^{2} / 100(t-s)}(t-s)^{-3 / 2}\left(\left|\widehat{\chi}_{j}\left(\frac{1}{4} L, s\right)\right|+\left|\widehat{\chi}_{j}\left(-\frac{1}{4} L, s\right)\right|\right) d s,
\end{aligned}
$$

and hence

$$
\begin{aligned}
\left|\widehat{\chi}_{j}(z, t)\right| \leqslant & \left(\frac{1}{4} L\right)^{-\left(\nu_{j}+2\right) / 2}+C L^{21} \int_{-L / 4}^{t} e^{-L^{2} / 100(t-s)}(t-s)^{-3 / 2}(-s)^{-\left(\nu_{j}+2\right) / 2} d s \\
\leqslant & C\left(\frac{1}{4} L\right)^{-\left(\nu_{j}+2\right) / 2}+C L^{20} \int_{-L / 4}^{t} e^{-L^{2} / 200(t-s)}(-s)^{-\left(\nu_{j}+2\right) / 2} d s \\
\leqslant & C\left(\frac{1}{4} L\right)^{-\left(\nu_{j}+2\right) / 2}+C L^{20} \int_{-L / 4}^{\left(1+1 / \sqrt{\nu_{j}}\right) t} e^{-L^{2} / 200(t-s)}(-s)^{-\left(\nu_{j}+2\right) / 2} d s \\
& +C L^{20} \int_{\left(1+1 / \sqrt{\nu_{j}}\right) t}^{t} e^{-L^{2} / 200(t-s)}(-s)^{-\left(\nu_{j}+2\right) / 2} d s \\
\leqslant & \left(\frac{1}{4} L\right)^{-\left(\nu_{j}+2\right) / 2}+C L^{20} e^{-L / 100}\left(1+\frac{1}{\sqrt{\nu_{j}}}\right)^{-\nu_{j} / 2}(-t)^{-\nu_{j} / 2} \\
& +C L^{20} e^{-L^{2} \sqrt{\nu_{j}} / 200(-t)}(-t)^{-\nu_{j} / 2}
\end{aligned}
$$

for all $t \in[-1000,-1]$ and all $z \in[-1000,1000]$. Therefore,

$$
\left|\chi_{j}(z, t)\right| \leqslant C\left(\frac{L}{4(-t)}\right)^{-\left(\nu_{j}+2\right) / 2}+C L^{20} e^{-L / 100}\left(1+\frac{1}{\sqrt{\nu_{j}}}\right)^{-\nu_{j} / 2}+C L^{20} e^{-L^{2} \sqrt{\nu_{j}} / 200(-t)}
$$

for all $t \in[-1000,-1]$ and all $z \in[-1000,1000]$. Summation over $j$ gives

$$
|\chi(z, t)|_{g_{S^{2}}}=\left|\sum_{j=1}^{\infty} \chi_{j}(z, t) S_{j}\right|_{g_{S^{2}}} \leqslant C \sum_{j=1}^{\infty} \nu_{j}\left|\chi_{j}(z, t)\right| \leqslant C L^{-1}
$$

in the region $\{|z| \leqslant 1000,-1000 \leqslant t \leqslant-1\}$. 
Step 2. We next analyze the equation for $\sigma(z, t)$. Let $Q_{j}, j=1,2, \ldots$, denote the eigenfunctions of the Laplacian on vector fields on $S^{2}$, so that $\Delta_{S^{2}} Q_{j}=-\mu_{j} Q_{j}$. By $[8$, Proposition A.1], the eigenvalues satisfy $\mu_{j} \geqslant 1$. We assume that the eigenfunctions $Q_{j}$ are normalized so that $\int_{S^{2}}\left|Q_{j}\right|_{g_{S^{2}}}^{2} d \operatorname{vol}_{S^{2}}=1$ for each $j$. Then,

$$
\sup _{S^{2}}\left|Q_{j}\right|_{g_{S^{2}}} \leqslant C\left\|Q_{j}\right\|_{H^{2}} \leqslant C \mu_{j}
$$

for each $j$. Moreover, $\mu_{j} \sim j$ as $j \rightarrow \infty$ (cf. [7, Corollary 2.43]). Let us write

$$
\sigma(z, t)=\sum_{j=1}^{\infty} \sigma_{j}(z, t) Q_{j}
$$

where

$$
\sigma_{j}(z, t)=\int_{S^{2}}\left\langle\sigma(z, t), Q_{j}\right\rangle_{g_{S^{2}}} d \operatorname{vol}_{S^{2}}
$$

Note that $\left|\sigma_{j}(z, t)\right| \leqslant C \sup _{S^{2}}|\sigma(z, t)|_{g_{S^{2}}}$. Moreover, the function $\sigma_{j}(z, t)$ satisfies

$$
\frac{\partial}{\partial t} \sigma_{j}(z, t)=\frac{\partial^{2}}{\partial z^{2}} \sigma_{j}(z, t)-\frac{\mu_{j}+1}{-2 t} \sigma_{j}(z, t)
$$

Hence, the function $\hat{\sigma}_{j}(z, t):=(-t)^{-\left(\mu_{j}+1\right) / 2} \sigma_{j}(z, t)$ satisfies

$$
\frac{\partial}{\partial t} \hat{\sigma}_{j}(z, t)=\frac{\partial^{2}}{\partial z^{2}} \hat{\sigma}_{j}(z, t) .
$$

Moreover, $\left|\hat{\sigma}_{j}(z, t)\right| \leqslant C(-t)^{-\mu_{j} / 2}$ in the region $\left\{|z| \leqslant \frac{1}{2} L,-\frac{1}{2} L \leqslant t \leqslant-\frac{1}{4} L\right\}$, and

$$
\left|\hat{\sigma}_{j}(z, t)\right| \leqslant C L^{20}(-t)^{-\left(\mu_{j}+2\right) / 2}
$$

in the region $\left\{|z| \leqslant \frac{1}{2} L,-\frac{1}{4} L \leqslant t \leqslant-1\right\}$. Using the solution formula for the Dirichlet problem for the 1 -dimensional heat equation on the rectangle $\left[-\frac{1}{4} L, \frac{1}{4} L\right] \times\left[-\frac{1}{4} L,-1\right]$, we obtain

$$
\begin{aligned}
\left|\hat{\sigma}_{j}(z, t)\right| \leqslant C & \sup _{z \in[-L / 4, L / 4]}\left|\hat{\sigma}_{j}\left(z,-\frac{1}{4} L\right)\right| \\
& +C L \int_{-L / 4}^{t} e^{-L^{2} / 100(t-s)}(t-s)^{-3 / 2}\left(\left|\hat{\sigma}_{j}\left(\frac{1}{4} L, s\right)\right|+\left|\hat{\sigma}_{j}\left(-\frac{1}{4} L, s\right)\right|\right) d s,
\end{aligned}
$$


and hence

$$
\begin{aligned}
\left|\hat{\sigma}_{j}(z, t)\right| \leqslant & C\left(\frac{1}{4} L\right)^{-\mu_{j} / 2}+C L^{21} \int_{-L / 4}^{t} e^{-L^{2} / 100(t-s)}(t-s)^{-3 / 2}(-s)^{-\left(\mu_{j}+2\right) / 2} d s \\
\leqslant & C\left(\frac{1}{4} L\right)^{-\mu_{j} / 2}+C L^{20} \int_{-L / 4}^{t} e^{-L^{2} / 200(t-s)}(-s)^{-\left(\mu_{j}+2\right) / 2} d s \\
\leqslant & C\left(\frac{1}{4} L\right)^{-\mu_{j} / 2}+C L^{20} \int_{-L / 4}^{\left(1+1 / \sqrt{\left.\mu_{j}\right) t}\right.} e^{-L^{2} / 200(t-s)}(-s)^{-\left(\mu_{j}+2\right) / 2} d s \\
& +C L^{20} \int_{\left(1+1 / \sqrt{\mu_{j}}\right) t}^{t} e^{-L^{2} / 200(t-s)}(-s)^{-\left(\mu_{j}+2\right) / 2} d s \\
\leqslant & \left(\frac{1}{4} L\right)^{-\mu_{j} / 2}+C L^{20} e^{-L / 100}\left(1+\frac{1}{\sqrt{\mu_{j}}}\right)^{-\mu_{j} / 2}(-t)^{-\mu_{j} / 2} \\
& +C L^{20} e^{-L^{2} \sqrt{\mu_{j}} / 200(-t)}(-t)^{-\mu_{j} / 2}
\end{aligned}
$$

for all $t \in[-1000,-1]$ and all $z \in[-1000,1000]$. Therefore,

$$
\left|\sigma_{j}(z, t)\right| \leqslant C\left(\frac{L}{4(-t)}\right)^{-\mu_{j} / 2}+C L^{20} e^{-L / 100}\left(1+\frac{1}{\sqrt{\mu_{j}}}\right)^{-\mu_{j} / 2}+C L^{20} e^{-L^{2} \sqrt{\mu_{j}} / 200(-t)}
$$

for all $t \in[-1000,-1]$ and all $z \in[-1000,1000]$. Summation over $j$ gives

$$
|\sigma(z, t)|_{g_{S^{2}}}=\left|\sum_{j=1}^{\infty} \sigma_{j}(z, t) Q_{j}\right|_{g_{S^{2}}} \leqslant C \sum_{j=1}^{\infty} \mu_{j}\left|\sigma_{j}(z, t)\right| \leqslant C L^{-1 / 2}
$$

for all $t \in[-1000,-1]$ and all $z \in[-1000,1000]$.

Step 3. We next analyze the equation for $\beta(z, t)$. Let $Y_{j}, j=0,1,2, \ldots$, denote the eigenfunctions of the Laplacian on scalar functions on $S^{2}$, so that $\Delta_{S^{2}} Y_{j}=-\lambda_{j} Y_{j}$. Note that $\lambda_{0}=0$ and $\lambda_{1}=2$. We assume that the eigenfunctions $Y_{j}$ are normalized so that $\int_{S^{2}} Y_{j}^{2} d \mathrm{vol}_{S^{2}}=1$. Then, $\sup _{S^{2}}\left|Y_{j}\right| \leqslant C\left\|Y_{j}\right\|_{H^{2}} \leqslant C \lambda_{j}$ for $j \geqslant 1$. Moreover, $\lambda_{j} \sim j$ as $j \rightarrow \infty$. Let us write

$$
\beta(z, t)=\sum_{j=0}^{\infty} \beta_{j}(z, t) Y_{j}
$$

where

$$
\beta_{j}(z, t)=\int_{S^{2}} \beta(z, t) Y_{j} d \operatorname{vol}_{S^{2}} .
$$

Note that $\left|\beta_{j}(z, t)\right| \leqslant C \sup _{S^{2}}|\beta(z, t)|$. Moreover, the function $\beta_{j}(z, t)$ satisfies

$$
\frac{\partial}{\partial t} \beta_{j}(z, t)=\frac{\partial^{2}}{\partial z^{2}} \beta_{j}(z, t)-\frac{\lambda_{j}}{-2 t} \beta_{j}(z, t) .
$$


Hence, the function $\hat{\beta}_{j}(z, t):=(-t)^{-\lambda_{j} / 2} \beta_{j}(z, t)$ satisfies

$$
\frac{\partial}{\partial t} \hat{\beta}_{j}(z, t)=\frac{\partial^{2}}{\partial z^{2}} \hat{\beta}_{j}(z, t)
$$

In the following, we consider modes with $j \geqslant 1$, so that $\lambda_{j} \geqslant 2$. By assumption,

$$
\left|\hat{\beta}_{j}(z, t)\right| \leqslant C(-t)^{-\lambda_{j} / 2}
$$

in the region $\left\{|z| \leqslant \frac{1}{2} L,-\frac{1}{2} L \leqslant t \leqslant-\frac{1}{4} L\right\}$, and

$$
\left|\hat{\beta}_{j}(z, t)\right| \leqslant C L^{20}(-t)^{-\left(\lambda_{j}+2\right) / 2}
$$

in the region $\left\{|z| \leqslant \frac{1}{2} L,-\frac{1}{4} L \leqslant t \leqslant-1\right\}$. The solution formula for the Dirichlet problem for the 1 -dimensional heat equation on the rectangle $\left[-\frac{1}{4} L, \frac{1}{4} L\right] \times\left[-\frac{1}{4} L,-1\right]$ implies

$$
\begin{aligned}
\left|\hat{\beta}_{j}(z, t)\right| \leqslant C & \sup _{z \in[-L / 4, L / 4]}\left|\hat{\beta}_{j}\left(z,-\frac{1}{4} L\right)\right| \\
& +C L \int_{-L / 4}^{t} e^{-L^{2} / 100(t-s)}(t-s)^{-3 / 2}\left(\left|\hat{\beta}_{j}\left(\frac{1}{4} L, s\right)\right|+\left|\hat{\beta}_{j}\left(-\frac{1}{4} L, s\right)\right|\right) d s
\end{aligned}
$$

for all $t \in[-1000,-1], z \in[-1000,1000]$, and $j \geqslant 1$. Therefore, we obtain

$$
\begin{aligned}
\left|\hat{\beta}_{j}(z, t)\right| \leqslant C & \left(\frac{1}{4} L\right)^{-\lambda_{j} / 2}+C L^{21} \int_{-L / 4}^{t} e^{-L^{2} / 100(t-s)}(t-s)^{-3 / 2}(-s)^{-\left(\lambda_{j}+2\right) / 2} d s \\
\leqslant & C\left(\frac{1}{4} L\right)^{-\lambda_{j} / 2}+C L^{20} \int_{-L / 4}^{t} e^{-L^{2} / 200(t-s)}(-s)^{-\left(\lambda_{j}+2\right) / 2} d s \\
\leqslant & C\left(\frac{1}{4} L\right)^{-\lambda_{j} / 2}+C L^{20} \int_{-L / 4}^{\left(1+1 / \sqrt{\lambda_{j}}\right) t} e^{-L^{2} / 200(t-s)}(-s)^{-\left(\lambda_{j}+2\right) / 2} d s \\
& +C L^{20} \int_{\left(1+1 / \sqrt{\lambda_{j}}\right) t}^{t} e^{-L^{2} / 200(t-s)}(-s)^{-\left(\lambda_{j}+2\right) / 2} d s \\
\leqslant & \left(\frac{1}{4} L\right)^{-\lambda_{j} / 2}+C L^{20} e^{-L / 100}\left(1+\frac{1}{\sqrt{\lambda_{j}}}\right)^{-\lambda_{j} / 2}(-t)^{-\lambda_{j} / 2} \\
& +C L^{20} e^{-L^{2} \sqrt{\lambda_{j}} / 200(-t)}(-t)^{-\lambda_{j} / 2}
\end{aligned}
$$

for all $t \in[-1000,-1], z \in[-1000,1000]$, and $j \geqslant 1$. Consequently,

$$
\left|\beta_{j}(z, t)\right| \leqslant C\left(\frac{L}{4(-t)}\right)^{-\lambda_{j} / 2}+C L^{20} e^{-L / 100}\left(1+\frac{1}{\sqrt{\lambda_{j}}}\right)^{-\lambda_{j} / 2}+C L^{20} e^{-L^{2} \sqrt{\lambda_{j}} / 200(-t)}
$$


for all $t \in[-1000,-1], z \in[-1000,1000]$, and $j \geqslant 1$. Summation over $j \geqslant 1$ gives

$$
|\beta(z, t)-\bar{\beta}(z, t)|=\left|\sum_{j=1}^{\infty} \beta_{j}(z, t) Y_{j}\right| \leqslant C \sum_{j=1}^{\infty} \lambda_{j}\left|\beta_{j}(z, t)\right| \leqslant C L^{-1}
$$

for all $t \in[-1000,-1]$ and all $z \in[-1000,1000]$.

Step 4 . We finally analyze the equation for $\omega(z, t)$. As above, let $Y_{j}, j=0,1,2, \ldots$, denote the eigenfunctions of the Laplacian on scalar functions on $S^{2}$, so that

$$
\Delta_{S^{2}} Y_{j}=-\lambda_{j} Y_{j} .
$$

Note that $\lambda_{0}=0, \lambda_{1}=\lambda_{2}=\lambda_{3}=2$, and $\lambda_{4}=6$. We write

$$
\omega(z, t)=\sum_{j=0}^{\infty} \omega_{j}(z, t) Y_{j},
$$

where

$$
\omega_{j}(z, t)=\int_{S^{2}} \omega(z, t) Y_{j} d \mathrm{vol}_{S^{2}} .
$$

Note that $\left|\omega_{j}(z, t)\right| \leqslant C \sup _{S^{2}}|\omega(z, t)|$. Moreover, the function $\omega_{j}(z, t)$ satisfies

$$
\frac{\partial}{\partial t} \omega_{j}(z, t)=\frac{\partial^{2}}{\partial z^{2}} \omega_{j}(z, t)-\frac{\lambda_{j}}{-2 t} \omega_{j}(z, t) .
$$

Hence, the function $\widehat{\omega}_{j}(z, t):=(-t)^{-\lambda_{j} / 2} \omega_{j}(z, t)$ satisfies

$$
\frac{\partial}{\partial t} \widehat{\omega}_{j}(z, t)=\frac{\partial^{2}}{\partial z^{2}} \widehat{\omega}_{j}(z, t) .
$$

In the following, we consider modes with $j \geqslant 1$. We break the discussion into two subcases.

- Suppose first that $j \geqslant 4$, so that $\lambda_{j} \geqslant 6$. By assumption,

$$
\left|\widehat{\omega}_{j}(z, t)\right| \leqslant C(-t)^{-\left(\lambda_{j}-2\right) / 2}
$$

in the region $\left\{|z| \leqslant \frac{1}{2} L,-\frac{1}{2} L \leqslant t \leqslant-\frac{1}{4} L\right\}$, and

$$
\left|\widehat{\omega}_{j}(z, t)\right| \leqslant C L^{20}(-t)^{-\left(\lambda_{j}+2\right) / 2}
$$

in the region $\left\{|z| \leqslant \frac{1}{2} L,-\frac{1}{4} L \leqslant t \leqslant-1\right\}$. The solution formula for the 1-dimensional heat equation on the rectangle $\left[-\frac{1}{4} L, \frac{1}{4} L\right] \times\left[-\frac{1}{4} L,-1\right]$ implies that

$$
\begin{aligned}
\left|\widehat{\omega}_{j}(z, t)\right| \leqslant C & \sup _{z \in[-L / 4, L / 4]}\left|\widehat{\omega}_{j}\left(z,-\frac{1}{4} L\right)\right| \\
& +C L \int_{-L / 4}^{t} e^{-L^{2} / 100(t-s)}(t-s)^{-3 / 2}\left(\left|\widehat{\omega}_{j}\left(\frac{1}{4} L, s\right)\right|+\left|\widehat{\omega}_{j}\left(-\frac{1}{4} L, s\right)\right|\right) d s
\end{aligned}
$$


for all $t \in[-1000,-1], z \in[-1000,1000]$, and $j \geqslant 4$. Therefore,

$$
\begin{aligned}
\left|\widehat{\omega}_{j}(z, t)\right| \leqslant C & \left(\frac{1}{4} L\right)^{-\left(\lambda_{j}-2\right) / 2}+C L^{20} e^{-L / 100}\left(1+\frac{1}{\sqrt{\lambda_{j}}}\right)^{-\lambda_{j} / 2}(-t)^{-\lambda_{j} / 2} \\
& +C L^{20} e^{-L^{2} \sqrt{\lambda_{j}} / 200(-t)}(-t)^{-\lambda_{j} / 2}
\end{aligned}
$$

for all $t \in[-1000,-1], z \in[-1000,1000]$, and $j \geqslant 4$. Consequently,

$$
\left|\omega_{j}(z, t)\right| \leqslant C\left(\frac{L}{4(-t)}\right)^{-\left(\lambda_{j}-2\right) / 2}+C L^{20} e^{-L / 100}\left(1+\frac{1}{\sqrt{\lambda_{j}}}\right)^{-\lambda_{j} / 2}+C L^{20} e^{-L^{2} \sqrt{\lambda_{j}} / 200(-t)}
$$

for all $t \in[-1000,-1], z \in[-1000,1000]$, and $j \geqslant 4$. Summation over $j \geqslant 4$ gives

$$
\left|\sum_{j=4}^{\infty} \omega_{j}(z, t) Y_{j}\right| \leqslant C \sum_{j=4}^{\infty} \lambda_{j}\left|\omega_{j}(z, t)\right| \leqslant C L^{-2}
$$

for all $t \in[-1000,-1]$ and all $z \in[-1000,1000]$.

- Suppose finally that $1 \leqslant j \leqslant 3$, so that $\lambda_{j}=2$. In this case, $\left|\widehat{\omega}_{j}(z, t)\right| \leqslant C$ in the region $\left\{|z| \leqslant \frac{1}{2} L,-\frac{1}{2} L \leqslant t \leqslant-\frac{1}{4} L\right\}$, and $\left|\widehat{\omega}_{j}(z, t)\right| \leqslant C L^{20}$ in the region $\left\{|z| \leqslant \frac{1}{2} L,-\frac{1}{4} L \leqslant t \leqslant-1\right\}$. Using standard interior estimates for the linear heat equation, we obtain

$$
\left|\frac{\partial}{\partial z} \widehat{\omega}_{j}(z, t)\right| \leqslant C(-t)^{-1 / 2}
$$

in the region $\left\{|z| \leqslant \frac{1}{4} L, t=-\frac{1}{4} L\right\}$, and

$$
\left|\frac{\partial}{\partial z} \widehat{\omega}_{j}(z, t)\right| \leqslant C L^{20}(-t)^{-1 / 2}
$$

in the region $\left\{|z| \leqslant \frac{1}{4} L,-\frac{1}{4} L \leqslant t \leqslant-1\right\}$. The solution formula for the 1-dimensional heat equation on the rectangle $\left[-\frac{1}{4} L, \frac{1}{4} L\right] \times\left[-\frac{1}{4} L,-1\right]$ implies that

$$
\begin{aligned}
& \quad\left|\frac{\partial}{\partial z} \widehat{\omega}_{j}(z, t)\right| \\
& \quad \leqslant C \sup _{z \in[-L / 4, L / 4]}\left|\frac{\partial}{\partial z} \widehat{\omega}_{j}\left(z,-\frac{1}{4} L\right)\right| \\
& \quad+C L \int_{-L / 4}^{t} e^{-L^{2} / 100(t-s)}(t-s)^{-3 / 2}\left(\left|\frac{\partial}{\partial z} \widehat{\omega}_{j}\left(\frac{1}{4} L, s\right)\right|+\left|\frac{\partial}{\partial z} \widehat{\omega}_{j}\left(-\frac{1}{4} L, s\right)\right|\right) d s
\end{aligned}
$$

for all $t \in[-2000,-1], z \in[-2000,2000]$, and $1 \leqslant j \leqslant 3$. Therefore,

$$
\left|\frac{\partial}{\partial z} \widehat{\omega}_{j}(z, t)\right| \leqslant C L^{-1 / 2}
$$


for all $t \in[-2000,-1], z \in[-2000,2000]$, and $1 \leqslant j \leqslant 3$. Using standard interior estimates for the linear heat equation, we obtain

$$
\left|\frac{\partial^{2}}{\partial z^{2}} \widehat{\omega}_{j}(z, t)\right| \leqslant C L^{-1 / 2}
$$

for all $t \in[-1000,-1], z \in[-1000,1000]$, and $1 \leqslant j \leqslant 3$. This implies

$$
\left|\frac{\partial}{\partial t} \widehat{\omega}_{j}(z, t)\right| \leqslant C L^{-1 / 2}
$$

for all $t \in[-1000,-1], z \in[-1000,1000]$, and $1 \leqslant j \leqslant 3$. Consequently, for each $1 \leqslant j \leqslant 3$, there exists a constant $q_{j}$ such that

$$
\left|\widehat{\omega}_{j}(z, t)-q_{j}\right| \leqslant C L^{-1 / 2}
$$

for all $t \in[-1000,-1]$ and all $z \in[-1000,1000]$. Note that $q_{j}$ is independent of $t$ and $z$. Thus, we conclude that

$$
\left|\omega_{j}(z, t)-(-t) q_{j}\right| \leqslant C L^{-1 / 2}
$$

for all $t \in[-1000,-1], z \in[-1000,1000]$, and $1 \leqslant j \leqslant 3$.

Putting these facts together, we conclude that

$$
\begin{aligned}
\mid \omega(z, t) & -\bar{\omega}(z, t)-(-t)\left(q_{1} Y_{1}+q_{2} Y_{2}+q_{3} Y_{3}\right) \mid \\
& =\left|\sum_{j=1}^{3}\left(\omega_{j}(z, t)-(-t) q_{j}\right) Y_{j}+\sum_{j=4}^{\infty} \omega_{j}(z, t) Y_{j}\right| \leqslant C L^{-1 / 2}
\end{aligned}
$$

for all $t \in[-1000,-1]$ and all $z \in[-1000,1000]$.

To summarize, we have shown that

$$
\begin{aligned}
& \left|h(t)-\bar{\omega}(z, t) g_{S^{2}}-\bar{\beta}(z, t) d z \otimes d z-(-t)\left(q_{1} Y_{1}+q_{2} Y_{2}+q_{3} Y_{3}\right) g_{S^{2}}\right|_{\bar{g}(t)} \\
& \leqslant C\left|\omega(z, t)-\bar{\omega}(z, t)-(-t)\left(q_{1} Y_{1}+q_{2} Y_{2}+q_{3} Y_{3}\right)\right| \\
& \quad+C|\chi(z, t)|_{g_{S^{2}}}+C|\sigma(z, t)|_{g_{S^{2}}}+C|\beta(z, t)-\bar{\beta}(z, t)| \\
& \leqslant C L^{-1 / 2}
\end{aligned}
$$

in the region $\{|z| \leqslant 1000,-1000 \leqslant t \leqslant-1\}$. Hence, if we define

$$
\psi:=q_{1} Y_{1}+q_{2} Y_{2}+q_{3} Y_{3},
$$

then the assertion follows. 


\section{Gluing approximate Killing vector fields}

Lemma 7.1. Let $U$ be a vector field on a Riemannian manifold, and let $\gamma$ be a unit-speed geodesic. Then,

$$
\left|D_{s} D_{s} U+g^{k m} R\left(\gamma^{\prime}(s), \partial_{k}, \gamma^{\prime}(s), U\right) \partial_{m}\right| \leqslant C\left|D\left(\mathscr{L}_{U}(g)\right)\right|
$$

along $\gamma$.

Proof. We compute

$$
\begin{aligned}
& \left|D_{i} D_{j} U_{k}+R_{i k j l} U^{l}+D_{j} D_{i} U_{k}+R_{j k i l} U^{l}\right| \\
& \quad=\left|D_{i}\left(D_{j} U_{k}+D_{k} U_{j}\right)+D_{j}\left(D_{i} U_{k}+D_{k} U_{i}\right)-D_{k}\left(D_{i} U_{j}+D_{j} U_{i}\right)\right| \\
& \quad \leqslant C\left|D\left(\mathscr{L}_{U}(g)\right)\right| .
\end{aligned}
$$

From this, the assertion easily follows.

LEMma 7.2. Let $\bar{g}$ denote the standard metric on the cylinder $S^{2} \times[-20,20]$ with scalar curvature 1 , and let $g$ be a Riemannian metric which is close to $\bar{g}$ in $C^{10}$. Let $\bar{x}$ be a point on the center slice $S^{2} \times\{0\}$. Suppose that $U$ is a vector field satisfying $\sup _{B_{g}(\bar{x}, 12)}\left|D\left(\mathscr{L}_{U}(g)\right)\right| \leqslant 1$ and $|U|+|D U| \leqslant 1$ at $\bar{x}$. Then, $\sup _{B_{g}(\bar{x}, 12)}|U| \leqslant C$.

Proof. Let $\gamma$ be a unit-speed geodesic emanating from $\bar{x}$ with length at most 12 . By Lemma 7.1,

$$
\left|D_{s} D_{s} U+g^{k m} R\left(\gamma^{\prime}(s), \partial_{k}, \gamma^{\prime}(s), U\right) \partial_{m}\right| \leqslant C
$$

along $\gamma$. Since $|U|+|D U| \leqslant 1$ at $\bar{x}$, we conclude that $|U| \leqslant C$ along $\gamma$.

LEMMA 7.3. Let $\bar{g}$ denote the standard metric on the cylinder $S^{2} \times[-20,20]$ with scalar curvature 1 , and let $g$ be a Riemannian metric which is close to $\bar{g}$ in $C^{10}$. Let $\bar{x}$ be a point on the center slice $S^{2} \times\{0\}$, and let $\Sigma$ denote the leaf of the CMC foliation with respect to $g$ which passes through $\bar{x}$. Suppose that $U$ is a vector field satisfying

$$
\sup _{B_{g}(\bar{x}, 12)}\left|\mathscr{L}_{U}(g)\right|+\left|D\left(\mathscr{L}_{U}(g)\right)\right| \leqslant 1 \quad \text { and } \quad \int_{\Sigma}|U|^{2} d \mu_{g} \leqslant 1 .
$$

Then,

$$
\sup _{B_{g}(\bar{x}, 12)}|U| \leqslant C .
$$

Proof. Suppose that the assertion is false. Then, there exist a sequence of metrics $g^{(j)}$ on $S^{2} \times[-20,20]$ and a sequence of vector fields $U^{(j)}$ such that $g^{(j)} \rightarrow \bar{g}$ in $C^{10}$,

$$
\begin{array}{r}
\sup _{B_{g^{(j)}}(\bar{x}, 12)}\left|\mathscr{L}_{U^{(j)}}\left(g^{(j)}\right)\right|+\left|D\left(\mathscr{L}_{U^{(j)}}\left(g^{(j)}\right)\right)\right| \leqslant 1, \\
\int_{\Sigma^{(j)}}\left|U^{(j)}\right|^{2} d \mu_{g^{(j)}} \leqslant 1,
\end{array}
$$


and $\sup _{B_{g^{(j)}}(\bar{x}, 12)}\left|U^{(j)}\right| \rightarrow \infty$. Here, $\Sigma^{(j)}$ denotes the slice of the CMC foliation with respect to $g^{(j)}$ which passes through $\bar{x}$. For each $j$, we define a real number $A_{j}$ so that $\left|U^{(j)}\right|+\left|D U^{(j)}\right|=A_{j}$ at $\bar{x}$. By Lemma 7.2 ,

$$
\sup _{B_{g}(j)(\bar{x}, 12)}\left|U^{(j)}\right| \leqslant C A_{j}+C .
$$

In particular, $A_{j} \rightarrow \infty$. Also, the estimate $\sup _{B_{g^{(j)}}(\bar{x}, 12)}\left|D\left(\mathscr{L}_{U^{(j)}}\left(g^{(j)}\right)\right)\right| \leqslant 1$ implies that

$$
\sup _{B_{g^{(j)}}(\bar{x}, 12)}\left|\Delta_{g^{(j)}} U^{(j)}+\operatorname{Ric}_{g^{(j)}}\left(U^{(j)}\right)\right| \leqslant C .
$$

Consequently, the rescaled vector fields $A_{j}^{-1} U^{(j)}$ converge in $C^{1,1 / 2}\left(B_{\bar{g}}(\bar{x}, 10)\right)$ to a vector field $U$. The limiting vector field $U$ satisfies $\mathscr{L}_{U}(\bar{g})=0$ and $\int_{\bar{\Sigma}}|U|^{2} d \mu_{\bar{g}}=0$. In other words, $U$ is a Killing vector field on the cylinder which vanishes along $\bar{\Sigma}$. Consequently, $U$ vanishes identically. On the other hand, $|U|+|D U|=1$ at $\bar{x}$. This is a contradiction.

PROPOSITION 7.4. If $\varepsilon_{0}$ is sufficiently small, then the following holds. Let $\bar{g}$ denote the standard metric on the cylinder $S^{2} \times[-20,20]$ with scalar curvature 1 , let $g$ be a Riemannian metric with $\|g-\bar{g}\|_{C^{10}} \leqslant \varepsilon_{0}$, and let $\varepsilon \leqslant \varepsilon_{0}$. Let $\bar{x}$ be a point on the center slice $S^{2} \times\{0\}$, and let $\Sigma$ denote the leaf of the CMC foliation with respect to $g$ which passes through $\bar{x}$. Suppose that $U^{(1)}, U^{(2)}$, and $U^{(3)}$ are vector fields with the following properties:

- $\sup _{B_{g}(\bar{x}, 12)} \sum_{a=1}^{3}\left|\mathscr{L}_{U^{(a)}}(g)\right|^{2}+\left|D\left(\mathscr{L}_{U^{(a)}}(g)\right)\right|^{2} \leqslant \varepsilon^{2} ;$

- $\sup _{\Sigma} \sum_{a=1}^{3}\left|\left\langle U^{(a)}, \nu\right\rangle\right|^{2} \leqslant \varepsilon^{2}$;

- $\sum_{a, b=1}^{3}\left|\delta_{a b}-\operatorname{area}_{g}(\Sigma)^{-2} \int_{\Sigma}\left\langle U^{(a)}, U^{(b)}\right\rangle d \mu_{g}\right|^{2} \leqslant \varepsilon^{2}$.

Moreover, suppose that $\widetilde{U}^{(1)}, \widetilde{U}^{(2)}$, and $\widetilde{U}^{(3)}$ are vector fields with the following properties:

- $\sup _{B_{g}(\bar{x}, 12)} \sum_{a=1}^{3}\left|\mathscr{L}_{\tilde{U}^{(a)}}(g)\right|^{2}+\left|D\left(\mathscr{L}_{\widetilde{U}^{(a)}}(g)\right)\right|^{2} \leqslant \varepsilon^{2} ;$

- $\sup _{\Sigma} \sum_{a=1}^{3}\left|\left\langle\widetilde{U}^{(a)}, \nu\right\rangle\right|^{2} \leqslant \varepsilon^{2}$

- $\sum_{a, b=1}^{3}\left|\delta_{a b}-\operatorname{area}_{g}(\Sigma)^{-2} \int_{\Sigma}\left\langle\widetilde{U}^{(a)}, \widetilde{U}^{(b)}\right\rangle d \mu_{g}\right|^{2} \leqslant \varepsilon^{2}$.

Then there exists a $3 \times 3$ matrix $\omega \in O(3)$ such that

$$
\sup _{B_{g}(\bar{x}, 9)} \sum_{a=1}^{3}\left|\sum_{b=1}^{3} \omega_{a b} U^{(b)}-\widetilde{U}^{(a)}\right|^{2} \leqslant C \varepsilon^{2} .
$$

Proof. Suppose that the assertion is false. Then, we can find a sequence of metrics $g^{(j)}$ on $S^{2} \times[-20,20]$, a collection of vector fields $U^{(1, j)}, U^{(2, j)}$, and $U^{(3, j)}$, a collection of vector fields $\widetilde{U}^{(1, j)}, \widetilde{U}^{(2, j)}$, and $\widetilde{U}^{(3, j)}$, and a sequence of positive numbers $\varepsilon_{j}$ with the following properties:

- $\left\|g^{(j)}-\bar{g}\right\|_{C^{10} \leqslant j^{-1}}$ and $\varepsilon_{j} \leqslant j^{-1}$; 
- $\sup _{B_{g^{(j)}(\bar{x}, 12)}} \sum_{a=1}^{3}\left|\mathscr{L}_{U^{(a, j)}}\left(g^{(j)}\right)\right|^{2}+\left|D\left(\mathscr{L}_{U^{(a, j)}}\left(g^{(j)}\right)\right)\right|^{2} \leqslant \varepsilon_{j}^{2}$;

- $\sup _{\Sigma^{(j)}} \sum_{a=1}^{3}\left|\left\langle U^{(a, j)}, \nu\right\rangle\right|^{2} \leqslant \varepsilon_{j}^{2}$;

- $\sum_{a, b=1}^{3}\left|\delta_{a b}-\operatorname{area}_{g}\left(\Sigma^{(j)}\right)^{-2} \int_{\Sigma^{(j)}}\left\langle U^{(a, j)}, U^{(b, j)}\right\rangle d \mu_{g^{(j)}}\right|^{2} \leqslant \varepsilon_{j}^{2}$;

- $\sup _{B_{g^{(j)}}(\bar{x}, 12)} \sum_{a=1}^{3}\left|\mathscr{L}_{\widetilde{U}^{(a, j)}}\left(g^{(j)}\right)\right|^{2}+\left|D\left(\mathscr{L}_{\widetilde{U}^{(a, j)}}\left(g^{(j)}\right)\right)\right|^{2} \leqslant \varepsilon_{j}^{2}$;

- $\sup _{\Sigma^{(j)}} \sum_{a=1}^{3}\left|\left\langle\widetilde{U}^{(a, j)}, \nu\right\rangle\right|^{2} \leqslant \varepsilon_{j}^{2}$;

- $\sum_{a, b=1}^{3}\left|\delta_{a b}-\operatorname{area}_{g}\left(\Sigma^{(j)}\right)^{-2} \int_{\Sigma^{(j)}}\left\langle\widetilde{U}^{(a, j)}, \widetilde{U}^{(b, j)}\right\rangle d \mu_{g^{(j)}}\right|^{2} \leqslant \varepsilon_{j}^{2}$;

- $\delta_{j}^{2}:=\inf _{\omega \in O(3)} \sup _{B_{g}(j)}(\bar{x}, 9) \sum_{a=1}^{3}\left|\sum_{b=1}^{3} \omega_{a b} U^{(b, j)}-\widetilde{U}^{(a, j)}\right|^{2} \geqslant\left(j \varepsilon_{j}\right)^{2}$.

Here, $\Sigma^{(j)}$ denotes the leaf of the CMC foliation with respect to $g^{(j)}$ which passes through $\bar{x}$.

Clearly,

$$
\int_{\Sigma^{(j)}} \sum_{a=1}^{3}\left|U^{(a, j)}\right|^{2} d \mu_{g^{(j)}} \leqslant C \text { and } \int_{\Sigma^{(j)}} \sum_{a=1}^{3}\left|\widetilde{U}^{(a, j)}\right|^{2} d \mu_{g^{(j)}} \leqslant C .
$$

Hence, Lemma 7.3 implies that

$$
\sup _{B_{g^{(j)}}(\bar{x}, 12)} \sum_{a=1}^{3}\left|U^{(a, j)}\right|^{2} \leqslant C \quad \text { and } \quad \sup _{B_{g^{(j)}}(\bar{x}, 12)} \sum_{a=1}^{3}\left|\widetilde{U}^{(a, j)}\right|^{2} \leqslant C .
$$

Moreover,

$$
\begin{array}{r}
\sup _{B_{g^{(j)}}(\bar{x}, 12)} \sum_{a=1}^{3}\left|\Delta_{g^{(j)}} U^{(a, j)}+\operatorname{Ric}_{g^{(j)}}\left(U^{(a, j)}\right)\right|^{2} \leqslant C \varepsilon_{j}^{2}, \\
\sup _{B_{g^{(j)}}(\bar{x}, 12)} \sum_{a=1}^{3}\left|\Delta_{g^{(j)}} \widetilde{U}^{(a, j)}+\operatorname{Ric}_{g^{(j)}}\left(\widetilde{U}^{(a, j)}\right)\right|^{2} \leqslant C \varepsilon_{j}^{2} .
\end{array}
$$

After passing to a subsequence, the vector fields $U^{(a, j)}$ converge in $C^{1,1 / 2}\left(B_{\bar{g}}(\bar{x}, 10)\right)$ to a vector field $U^{(a)}$ which satisfies $\mathscr{L}_{U^{(a)}}(\bar{g})=0$ and is tangential along $\bar{\Sigma}$. Similarly, the vector fields $\widetilde{U}^{(a, j)}$ converge in $C^{1,1 / 2}\left(B_{\bar{g}}(\bar{x}, 10)\right)$ to a vector field $\widetilde{U}^{(a)}$ which satisfies $\mathscr{L}_{\tilde{U}^{(a)}}(\bar{g})=0$ and is tangential along $\bar{\Sigma}$. Note that

$$
\operatorname{area}_{\bar{g}}(\bar{\Sigma})^{-2} \int_{\bar{\Sigma}}\left\langle U^{(a)}, U^{(b)}\right\rangle d \mu_{\bar{g}}=\operatorname{area}_{\bar{g}}(\bar{\Sigma})^{-2} \int_{\bar{\Sigma}}\left\langle\widetilde{U}^{(a)}, \widetilde{U}^{(b)}\right\rangle d \mu_{\bar{g}}=\delta_{a b} .
$$

Consequently, there exists a matrix $\bar{\omega} \in O(3)$ such that

$$
\widetilde{U}^{(a)}=\sum_{b=1}^{3} \bar{\omega}_{a b} U^{(b)}
$$

This implies $\delta_{j} \rightarrow 0$. 
For each $j$, we choose a $3 \times 3$ matrix $\omega^{(j)} \in O(3)$ such that

$$
\sup _{B_{g}(j)}(\bar{x}, 9) \sum_{a=1}^{3}\left|\sum_{b=1}^{3} \omega_{a b}^{(j)} U^{(b, j)}-\widetilde{U}^{(a, j)}\right|^{2}=\delta_{j}^{2} .
$$

Clearly, $\omega^{(j)} \rightarrow \bar{\omega}$ as $j \rightarrow \infty$. We next define

$$
V^{(a, j)}:=\delta_{j}^{-1}\left(\sum_{b=1}^{3} \omega_{a b}^{(j)} U^{(b, j)}-\widetilde{U}^{(a, j)}\right) .
$$

The vector fields $V^{(a, j)}$ have the following properties:

- $\sup _{B_{g^{(j)}}(\bar{x}, 9)} \sum_{a=1}^{3}\left|V^{(a, j)}\right|^{2}=1$;

- $\sup _{B_{g^{(j)}}(\bar{x}, 12)} \sum_{a=1}^{3}\left|\mathscr{L}_{V^{(a, j)}}\left(g^{(j)}\right)\right|^{2}+\left|D\left(\mathscr{L}_{V^{(a, j)}}\left(g^{(j)}\right)\right)\right|^{2} \leqslant C j^{-2}$;

- $\sup _{\Sigma^{(j)}} \sum_{a=1}^{3}\left|\left\langle V^{(a, j)}, \nu\right\rangle\right|^{2} \leqslant C j^{-2}$.

Using Lemma 7.3, we obtain $\sup _{B_{g}(j)}(\bar{x}, 12) \sum_{a=1}^{3}\left|V^{(a, j)}\right|^{2} \leqslant C$. Moreover,

$$
\sup _{B_{g^{(j)}}(\bar{x}, 12)} \sum_{a=1}^{3}\left|\Delta_{g^{(j)}} V^{(a, j)}+\operatorname{Ric}_{g^{(j)}}\left(V^{(a, j)}\right)\right|^{2} \leqslant C j^{-2} \text {. }
$$

Thus, after passing to a subsequence, the vector fields $V^{(a, j)}$ converge in $C^{1,1 / 2}\left(B_{\bar{g}}(\bar{x}, 10)\right)$ to a vector field $V^{(a)}$ which satisfies $\mathscr{L}_{V^{(a)}}(\bar{g})=0$ and is tangential along $\bar{\Sigma}$. Consequently, $V^{(a)}=\sum_{b=1}^{3} \sigma_{a b} \widetilde{U}^{(b)}$ for some $3 \times 3$-matrix $\sigma$.

We next observe that

$$
\begin{aligned}
\mid \delta_{j} & \int_{\Sigma^{(j)}}\left(\left\langle\widetilde{U}^{(a, j)}, V^{(b, j)}\right\rangle+\left\langle V^{(a, j)}, \widetilde{U}^{(b, j)}\right\rangle\right) d \mu_{g^{(j)}}+\delta_{j}^{2} \int_{\Sigma^{(j)}}\left\langle V^{(a, j)}, V^{(b, j)}\right\rangle d \mu_{g^{(j)}} \mid \\
& =\left|\int_{\Sigma^{(j)}}\left\langle\widetilde{U}^{(a, j)}+\delta_{j} V^{(a, j)}, \widetilde{U}^{(b, j)}+\delta_{j} V^{(b, j)}\right\rangle d \mu_{g^{(j)}}-\int_{\Sigma^{(j)}}\left\langle\widetilde{U}^{(a, j)}, \widetilde{U}^{(b, j)}\right\rangle d \mu_{g^{(j)}}\right| \\
& =\left|\sum_{c, d=1}^{3} \omega_{a c}^{(j)} \omega_{b d}^{(j)} \int_{\Sigma^{(j)}}\left\langle U^{(c, j)}, U^{(d, j)}\right\rangle d \mu_{g^{(j)}}-\int_{\Sigma^{(j)}}\left\langle\widetilde{U}^{(a, j)}, \widetilde{U}^{(b, j)}\right\rangle d \mu_{g^{(j)}}\right| \\
& \leqslant C \varepsilon_{j} .
\end{aligned}
$$

Since $\delta_{j} \rightarrow 0$ and $\delta_{j}^{-1} \varepsilon_{j} \leqslant j^{-1}$, we conclude that

$$
\int_{\bar{\Sigma}}\left(\left\langle\widetilde{U}^{(a)}, V^{(b)}\right\rangle+\left\langle V^{(a)}, \widetilde{U}^{(b)}\right\rangle\right) d \mu_{\bar{g}}=0 .
$$

Consequently, $\sigma$ is an anti-symmetric matrix. Let $\widetilde{\omega}^{(j)}:=\exp \left(-\delta_{j} \sigma\right) \omega^{(j)} \in O(3)$. Since $V^{(a)}=\sum_{b=1}^{3} \sigma_{a b} \widetilde{U}^{(b)}$, we obtain

$$
\sup _{B_{g}(j)}(\bar{x}, 9) \sum_{a=1}^{3}\left|V^{(a, j)}+\delta_{j}^{-1} \sum_{b=1}^{3}\left(\widetilde{\omega}_{a b}^{(j)}-\omega_{a b}^{(j)}\right) U^{(b, j)}\right|^{2} \rightarrow 0
$$


as $j \rightarrow \infty$. On the other hand, it follows from the definition of $\delta_{j}$ that

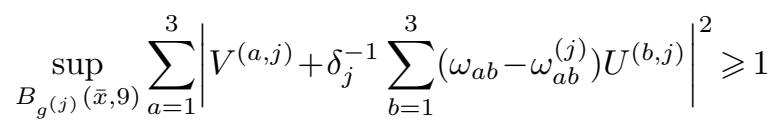

for each $j$ and each $\omega \in O(3)$. This is a contradiction.

Corollary 7.5. Let $\bar{g}$ denote the standard metric on the cylinder $S^{2} \times[-20,20]$ with scalar curvature 1 , let $g$ be a Riemannian metric with $\|g-\bar{g}\|_{C^{10}} \leqslant \varepsilon_{0}$, and let $\varepsilon \leqslant \varepsilon_{0}$. Moreover, suppose that $U^{(1)}, U^{(2)}$, and $U^{(3)}$, and $\widetilde{U}^{(1)}, \widetilde{U}^{(2)}$, and $\widetilde{U}^{(3)}$ are vector fields satisfying the assumptions of Proposition 7.4. Let $\eta$ be a smooth cut-off function such that $\eta=1$ on $S^{2} \times[-1000,-1]$ and $\eta=0$ on $S^{2} \times[1,1000]$. Then, there exists a $3 \times 3$ matrix $\omega \in O(3)$ with the property that the vector fields

$$
V^{(a)}:=\eta \sum_{b=1}^{3} \omega_{a b} U^{(b)}+(1-\eta) \widetilde{U}^{(a)}
$$

satisfy

$$
\sum_{a=1}^{3}\left|\mathscr{L}_{V^{(a)}}(g)\right|^{2}+\left|D\left(\mathscr{L}_{V^{(a)}}(g)\right)\right|^{2} \leqslant C \varepsilon^{2}
$$

in the transition region $S^{2} \times[-1,1]$.

Proof. By Proposition 7.4, we can find a $3 \times 3$ matrix $\omega \in O(3)$ with the property that the vector fields

$$
W^{(a)}:=\sum_{b=1}^{3} \omega_{a b} U^{(b)}-\widetilde{U}^{(a)} .
$$

satisfy $\sup _{B_{\bar{g}}(\bar{x}, 9)} \sum_{a=1}^{3}\left|W^{(a)}\right|^{2} \leqslant C \varepsilon^{2}$. Moreover,

$$
\sup _{B_{\bar{g}}(\bar{x}, 9)} \sum_{a=1}^{3}\left|\Delta W^{(a)}+\operatorname{Ric}\left(W^{(a)}\right)\right|^{2} \leqslant C \varepsilon^{2} .
$$

Using standard interior estimates for elliptic equations, we obtain

$$
\sup _{B_{\bar{g}}(\bar{x}, 8)}\left|D W^{(a)}\right|^{2} \leqslant C \varepsilon^{2} .
$$

We now define

$$
V^{(a)}:=\eta \sum_{b=1}^{3} \omega_{a b} U^{(b)}+(1-\eta) \widetilde{U}^{(a)}
$$


Then,

$$
\mathscr{L}_{V^{(a)}}(g)=\eta \sum_{b=1}^{3} \omega_{a b} \mathscr{L}_{U^{(b)}}(g)+(1-\eta) \mathscr{L}_{\tilde{U}^{(a)}}(g)+d \eta \otimes g\left(W^{(a)}, \cdot\right)+g\left(W^{(a)}, \cdot\right) \otimes d \eta .
$$

Using the estimate

$$
\sup _{B_{\bar{g}}(\bar{x}, 8)} \sum_{a=1}^{3}\left(\left|W^{(a)}\right|^{2}+\left|D W^{(a)}\right|^{2}\right) \leqslant C \varepsilon^{2},
$$

we conclude that

$$
\sup _{B_{\bar{g}}(\bar{x}, 8)} \sum_{a=1}^{3}\left(\left|\mathscr{L}_{V^{(a)}}(g)\right|^{2}+\left|D\left(\mathscr{L}_{V^{(a)}}(g)\right)\right|^{2}\right) \leqslant C \varepsilon^{2}
$$

Since the transition region $S^{2} \times[-1,1]$ is contained in $B_{\bar{g}}(\bar{x}, 8)$, the assertion follows.

\section{The neck improvement theorem}

Definition 8.1. Let $(M, g(t))$ be a solution to the Ricci flow in dimension 3, and let $(\bar{x}, \bar{t})$ be a point in space-time with $R(\bar{x}, \bar{t})=r^{-2}$. We say that $(\bar{x}, \bar{t})$ lies at the center of an evolving $\varepsilon$-neck if, after rescaling by the factor $r^{-1}$, the parabolic neighborhood $B_{g(\bar{t})}\left(\bar{x}, \varepsilon^{-1} r\right) \times\left[\bar{t}-\varepsilon^{-1} r^{2}, \bar{t}\right]$ is $\varepsilon$-close in $C^{\left[\varepsilon^{-1}\right]}$ to a family of shrinking cylinders.

Definition 8.2. Let $(M, g(t))$ be a solution to the Ricci flow in dimension 3, and let $(\bar{x}, \bar{t})$ be a point in space-time with $R(\bar{x}, \bar{t})=r^{-2}$. We assume that $(\bar{x}, \bar{t})$ lies at the center of an evolving $\varepsilon_{0}$-neck for some small number $\varepsilon_{0}$. We say that $(\bar{x}, \bar{t})$ is $\varepsilon$-symmetric if there exist smooth, time-independent vector fields $U^{(1)}, U^{(2)}$, and $U^{(3)}$ which are defined on an open set containing $\bar{B}_{g(\bar{t})}(\bar{x}, 100 r)$ and satisfy the following conditions:

- $\sup _{\bar{B}_{g(\bar{t})}(\bar{x}, 100 r) \times\left[\bar{t}-100 r^{2}, \bar{t}\right]} \sum_{l=0}^{2} \sum_{a=1}^{3} r^{2 l}\left|D^{l}\left(\mathscr{L}_{U^{(a)}}(g(t))\right)\right|^{2} \leqslant \varepsilon^{2}$;

- if $t \in\left[\bar{t}-100 r^{2}, \bar{t}\right]$ and $\Sigma \subset \bar{B}_{g(\bar{t})}(\bar{x}, 100 r)$ is a leaf of the CMC foliation of $(M, g(t))$, then $\sup _{\Sigma} \sum_{a=1}^{3} r^{-2}\left|\left\langle U^{(a)}, \nu\right\rangle\right|^{2} \leqslant \varepsilon^{2}$, where $\nu$ denotes the unit normal vector to $\Sigma$ in $(M, g(t))$;

- if $t \in\left[\bar{t}-100 r^{2}, \bar{t}\right]$ and $\Sigma \subset \bar{B}_{g(\bar{t})}(\bar{x}, 100 r)$ is a leaf of the CMC foliation of $(M, g(t))$, then

$$
\sum_{a, b=1}^{3}\left|\delta_{a b}-\operatorname{area}_{g(t)}(\Sigma)^{-2} \int_{\Sigma}\left\langle U^{(a)}, U^{(b)}\right\rangle_{g(t)} d \mu_{g(t)}\right|^{2} \leqslant \varepsilon^{2} .
$$

Lemma 8.3. Suppose that $(\bar{x}, \bar{t})$ is a point in space-time which is $\varepsilon$-symmetric. If $(\tilde{x}, \tilde{t})$ is sufficiently close to $(\bar{x}, \bar{t})$, then $(\tilde{x}, \tilde{t})$ is $2 \varepsilon$-symmetric. 
Proof. This follows immediately from the definition.

LEMMA 8.4. If $L$ is sufficiently large and $\varepsilon_{0}$ is sufficiently small depending on $L$, then the following holds. Let $(M, g(t))$ be a solution of the Ricci flow in dimension 3, and let $\left(x_{0},-1\right)$ be a point in space-time which lies at the center of an evolving $\varepsilon_{0}$ neck and satisfies $R\left(x_{0},-1\right)=1$. Moreover, we assume that every point in the parabolic neighborhood $B_{g(-1)}\left(x_{0}, L\right) \times[-L-1,-1)$ is $\varepsilon$-symmetric for some positive real number $\varepsilon \leqslant \varepsilon_{0}$. Then, given any $\bar{t} \in\left[-\frac{1}{10} L,-1\right]$, we can find time-independent vector fields $U^{(1)}$, $U^{(2)}$, and $U^{(3)}$ on $B_{g(-1)}\left(x_{0}, \frac{127}{128} L\right)$ with the following properties:

- $\sum_{a=1}^{3}\left|\mathscr{L}_{U^{(a)}}(g(t))\right|^{2}+(-t)\left|D\left(\mathscr{L}_{U^{(a)}}(g(t))\right)\right|^{2} \leqslant C \varepsilon^{2}$ on $B_{g(-1)}\left(x_{0}, \frac{127}{128} L\right) \times[10 \bar{t}, \bar{t}]$;

- $\sum_{a=1}^{3}(-t)^{-1}\left|\left\langle U^{(a)}, \nu\right\rangle\right|^{2} \leqslant C \varepsilon^{2}$ on $B_{g(-1)}\left(x_{0}, \frac{127}{128} L\right) \times[10 \bar{t}, \bar{t}]$, where $\nu$ denotes the unit normal vector to the CMC foliation of $(M, g(t))$;

- if $t \in[10 \bar{t}, \bar{t}]$ and $\Sigma \subset B_{g(-1)}\left(x_{0}, \frac{127}{128} L\right)$ is a leaf of the CMC foliation of $(M, g(t))$, then

$$
\sum_{a, b=1}^{3}\left|\delta_{a b}-\operatorname{area}_{g(t)}(\Sigma)^{-2} \int_{\Sigma}\left\langle U^{(a)}, U^{(b)}\right\rangle_{g(t)} d \mu_{g(t)}\right|^{2} \leqslant C \varepsilon^{2}
$$

Moreover, $U^{(1)}, U^{(2)}$, and $U^{(3)}$ are $C(L) \varepsilon_{0}$-close to the standard rotation vector fields on the cylinder in the $C^{2}$-norm.

Proof. We proceed in two steps.

Step 1. Suppose first that $\bar{t} \in\left[-\frac{1}{10} L,-1\right)$. By assumption, the point $(\bar{x}, \bar{t})$ is $\varepsilon$ symmetric whenever $\bar{x} \in B_{g(-1)}\left(x_{0}, L\right)$. By a repeated application of Corollary 7.5 , we can construct vector fields $U^{(1)}, U^{(2)}$, and $U^{(3)}$ satisfying the conditions above. Moreover, in view of Definition 8.2, the Lie derivatives $\mathscr{L}_{U^{(1)}}(g), \mathscr{L}_{U^{(2)}}(g)$, and $\mathscr{L}_{U^{(3)}}(g)$ are bounded by $C(L) \varepsilon$ in the $C^{2}$-norm. Consequently, the vector fields $U^{(1)}, U^{(2)}$, and $U^{(3)}$ are $C(L) \varepsilon_{0}$-close to the standard rotation vector fields on the cylinder in the $C^{2,1 / 2}$-norm.

Step 2. Suppose next that $\bar{t}=-1$. In this case, the assertion follows from the result in Step 1 by passing to the limit. Since the vector fields constructed in Step 1 are bounded in $C^{2,1 / 2}$, we may take the limit in $C^{2}$.

LEMMA 8.5. If $L$ is sufficiently large and $\varepsilon_{0}$ is sufficiently small depending on $L$, then the following holds. Let $(M, g(t))$ be a solution of the Ricci flow in dimension 3, and let $\left(x_{0},-1\right)$ be a point in space-time which lies at the center of an evolving $\varepsilon_{0}$-neck and satisfies $R\left(x_{0},-1\right)=1$. Consider a time $\bar{t} \in[-L,-1]$ and a positive real number $\varepsilon \leqslant \varepsilon_{0}$. Suppose that $U^{(1)}, U^{(2)}$, and $U^{(3)}$ are time-independent vector fields on $B_{g(-1)}\left(x_{0}, \frac{127}{128} L\right)$ with the following properties:

- $\sum_{a=1}^{3}\left|\mathscr{L}_{U^{(a)}}(g(\bar{t}))\right|^{2}+(-\bar{t})\left|D\left(\mathscr{L}_{U^{(a)}}(g(\bar{t}))\right)\right|^{2} \leqslant \varepsilon^{2}$ on $B_{g(-1)}\left(x_{0}, \frac{127}{128} L\right)$; 
- $\sum_{a=1}^{3}(-\bar{t})^{-1}\left|\left\langle U^{(a)}, \nu\right\rangle\right|^{2} \leqslant \varepsilon^{2}$ on $B_{g(-1)}\left(x_{0}, \frac{127}{128} L\right)$, where $\nu$ denotes the unit normal vector to the CMC foliation of $(M, g(\bar{t}))$;

- if $\Sigma \subset B_{g(-1)}\left(x_{0}, \frac{127}{128} L\right)$ is a leaf of the CMC foliation of $(M, g(\bar{t}))$, then

$$
\sum_{a, b=1}^{3}\left|\delta_{a b}-\operatorname{area}_{g(\bar{t})}(\Sigma)^{-2} \int_{\Sigma}\left\langle U^{(a)}, U^{(b)}\right\rangle_{g(\bar{t})} d \mu_{g(\bar{t})}\right|^{2} \leqslant \varepsilon^{2} .
$$

Moreover, suppose that $\widetilde{U}^{(1)}, \widetilde{U}^{(2)}$, and $\widetilde{U}^{(3)}$ are time-independent vector fields on $B_{g(-1)}\left(x_{0}, \frac{127}{128} L\right)$ with the following properties:

- $\sum_{a=1}^{3}\left|\mathscr{L}_{\widetilde{U}^{(a)}}(g(\bar{t}))\right|^{2}+(-\bar{t})\left|D\left(\mathscr{L}_{\widetilde{U}^{(a)}}(g(\bar{t}))\right)\right|^{2} \leqslant \varepsilon^{2}$ on $B_{g(-1)}\left(x_{0}, \frac{127}{128} L\right)$;

- $\sum_{a=1}^{3}(-\bar{t})^{-1}\left|\left\langle\widetilde{U}^{(a)}, \nu\right\rangle\right|^{2} \leqslant \varepsilon^{2}$ on $B_{g(-1)}\left(x_{0}, \frac{127}{128} L\right)$, where $\nu$ denotes the unit normal vector to the CMC foliation of $(M, g(\bar{t}))$;

- if $\Sigma \subset B_{g(-1)}\left(x_{0}, \frac{127}{128} L\right)$ is a leaf of the CMC foliation of $(M, g(\bar{t}))$, then

$$
\sum_{a, b=1}^{3}\left|\delta_{a b}-\operatorname{area}_{g(\bar{t})}(\Sigma)^{-2} \int_{\Sigma}\left\langle\widetilde{U}^{(a)}, \widetilde{U}^{(b)}\right\rangle_{g(\bar{t})} d \mu_{g(\bar{t})}\right|^{2} \leqslant \varepsilon^{2} .
$$

Then, there exists a $3 \times 3$ matrix $\omega \in O(3)$ such that

$$
\sup _{B_{g(-1)}\left(x_{0}, 31 L / 32\right)}(-\bar{t})^{-1} \sum_{a=1}^{3}\left|\sum_{b=1}^{3} \omega_{a b} U^{(b)}-\widetilde{U}^{(a)}\right|_{g(\bar{t})}^{2} \leqslant C L^{2} \varepsilon^{2} .
$$

Proof. By assumption, the flow is close to a family of shrinking cylinders. For each integer $m \in\left[-\frac{63}{64} L, \frac{63}{64} L\right]$, Proposition 7.4 implies that there exists a $3 \times 3$ matrix $\omega^{(m)} \in O(3)$ such that

$$
\sup _{S^{2} \times[m-1, m+1]}(-\bar{t})^{-1} \sum_{a=1}^{3}\left|\sum_{b=1}^{3} \omega_{a b}^{(m)} U^{(b)}-\widetilde{U}^{(a)}\right|_{g(\bar{t})}^{2} \leqslant C \varepsilon^{2} .
$$

From this, we deduce that $\left|\omega^{(m)}-\omega^{(m+1)}\right| \leqslant C \varepsilon$ for every integer $m$. Consequently, there exists a $3 \times 3$ matrix $\omega \in O(3)$ such that $\left|\omega^{(m)}-\omega\right| \leqslant C L \varepsilon$ for every integer $m \in\left[-\frac{63}{64} L, \frac{63}{64} L\right]$. This implies that

$$
\sup _{S^{2} \times[m-1, m+1]}(-\bar{t})^{-1} \sum_{a=1}^{3}\left|\sum_{b=1}^{3} \omega_{a b} U^{(b)}-\widetilde{U}^{(a)}\right|_{g(\bar{t})}^{2} \leqslant C L^{2} \varepsilon^{2}
$$

for every integer $m \in\left[-\frac{63}{64} L, \frac{63}{64} L\right]$.

We now state the main result of this section. 
THEOREM 8.6. (Neck improvement theorem) There exist a large constant $L$ and small positive constant $\varepsilon_{1}$ with the following property. Let $(M, g(t))$ be a solution of the Ricci flow in dimension 3 , and let $\left(x_{0}, t_{0}\right)$ be a point in space-time which lies at the center of an evolving $\varepsilon_{1}$-neck and satisfies $R\left(x_{0}, t_{0}\right)=r^{-2}$. Moreover, suppose that every point in the parabolic neighborhood $B_{g\left(t_{0}\right)}\left(x_{0}, L r\right) \times\left[t_{0}-L r^{2}, t_{0}\right)$ is $\varepsilon$-symmetric, where $\varepsilon \leqslant \varepsilon_{1}$. Then, $\left(x_{0}, t_{0}\right)$ is $\frac{1}{2} \varepsilon$-symmetric.

Proof. Throughout the proof, we will assume that $L$ is sufficiently large, and $\varepsilon_{1}$ is sufficiently small depending on $L$. Without loss of generality, we may assume that $t_{0}=-1$ and $R\left(x_{0},-1\right)=1$. In the parabolic neighborhood $B_{g(-1)}\left(x_{0}, L\right) \times[-L-1,-1]$, the metric $g(t)$ is $\varepsilon_{1}$-close to a family of shrinking cylinders in the $C^{100}$-norm. Let $\bar{g}(t)=(-2 t) g_{S^{2}}+d z \otimes d z$ denote the standard metric on the shrinking cylinders.

Step 1. Using Lemmas 8.4 and 8.5, we can construct time-dependent vector fields $U^{(1)}, U^{(2)}$, and $U^{(3)}$, defined on $B_{g(-1)}\left(x_{0}, \frac{15}{16} L\right) \times[-L,-1]$, with the following properties:

$$
\begin{cases}\frac{\partial}{\partial t} U^{(a)}=0 & \text { on } B_{g(-1)}\left(x_{0}, \frac{15}{16} L\right) \times\left[-L,-\frac{1}{4} L\right] \\ \left|\frac{\partial}{\partial t} U^{(a)}\right| \leqslant C L(-t)^{-1 / 2} \varepsilon & \text { on } B_{g(-1)}\left(x_{0}, \frac{15}{16} L\right) \times\left[-\frac{1}{4} L,-1\right]\end{cases}
$$

- $\left|\mathscr{L}_{U^{(a)}}(g(t))\right|+(-t)^{1 / 2}\left|D\left(\mathscr{L}_{U^{(a)}}(g(t))\right)\right| \leqslant C \varepsilon$ on $B_{g(-1)}\left(x_{0}, \frac{15}{16} L\right) \times[-L,-1]$.

Also, we can arrange that $U^{(1)}, U^{(2)}$, and $U^{(3)}$ are $C(L) \varepsilon_{1}$-close to the standard rotation vector fields on the cylinder in the $C^{2}$-norm. Note that

$$
\left|\Delta U^{(a)}+\operatorname{Ric}\left(U^{(a)}\right)\right| \leqslant C\left|D\left(\mathscr{L}_{U^{(a)}}(g)\right)\right| \leqslant C(-t)^{-1 / 2} \varepsilon
$$

on $B_{g(-1)}\left(x_{0}, \frac{15}{16} L\right) \times[-L,-1]$.

Step 2. Let $V^{(a)}$ denote the solution of the PDE

$$
\frac{\partial}{\partial t} V^{(a)}=\Delta V^{(a)}+\operatorname{Ric}\left(V^{(a)}\right)
$$

in the region $\left\{|z| \leqslant \frac{7}{8} L,-L \leqslant t \leqslant-1\right\}$ with Dirichlet boundary condition $V^{(a)}=U^{(a)}$ on the parabolic boundary $\left\{|z| \leqslant \frac{7}{8} L, t=-L\right\} \cup\left\{|z|=\frac{7}{8} L,-L \leqslant t \leqslant-1\right\}$. Note that the difference $V^{(a)}-U^{(a)}$ satisfies

$$
\begin{aligned}
\left|\frac{\partial}{\partial t}\left(V^{(a)}-U^{(a)}\right)-\Delta\left(V^{(a)}-U^{(a)}\right)-\operatorname{Ric}\left(V^{(a)}-U^{(a)}\right)\right| & =\left|\Delta U^{(a)}+\operatorname{Ric}\left(U^{(a)}\right)\right| \\
& \leqslant C(-t)^{-1 / 2} \varepsilon
\end{aligned}
$$


in the region $\left\{|z| \leqslant \frac{7}{8} L,-L \leqslant t \leqslant-\frac{1}{4} L\right\}$, and

$$
\begin{aligned}
\left|\frac{\partial}{\partial t}\left(V^{(a)}-U^{(a)}\right)-\Delta\left(V^{(a)}-U^{(a)}\right)-\operatorname{Ric}\left(V^{(a)}-U^{(a)}\right)\right| & \leqslant\left|\frac{\partial}{\partial t} U^{(a)}\right|+\left|\Delta U^{(a)}+\operatorname{Ric}\left(U^{(a)}\right)\right| \\
& \leqslant C L(-t)^{-1 / 2} \varepsilon
\end{aligned}
$$

in the region $\left\{|z| \leqslant \frac{7}{8} L,-\frac{1}{4} L \leqslant t \leqslant-1\right\}$. Hence, Proposition 5.3 implies that

$$
\frac{\partial}{\partial t}\left|V^{(a)}-U^{(a)}\right| \leqslant \Delta\left|V^{(a)}-U^{(a)}\right|+C(-t)^{-1 / 2} \varepsilon
$$

in the region $\left\{|z| \leqslant \frac{7}{8} L,-L \leqslant t \leqslant-\frac{1}{4} L\right\}$, and

$$
\frac{\partial}{\partial t}\left|V^{(a)}-U^{(a)}\right| \leqslant \Delta\left|V^{(a)}-U^{(a)}\right|+C L(-t)^{-1 / 2} \varepsilon
$$

in the region $\left\{|z| \leqslant \frac{7}{8} L,-\frac{1}{4} L \leqslant t \leqslant-1\right\}$. Using the maximum principle, we obtain

$$
\left|V^{(a)}-U^{(a)}\right| \leqslant C L^{1 / 2} \varepsilon
$$

in the region $\left\{|z| \leqslant \frac{7}{8} L,-L \leqslant t \leqslant-\frac{1}{4} L\right\}$, and

$$
\left|V^{(a)}-U^{(a)}\right| \leqslant C L^{2} \varepsilon
$$

in the region $\left\{|z| \leqslant \frac{7}{8} L,-\frac{1}{4} L \leqslant t \leqslant-1\right\}$. Standard interior estimates for linear parabolic equations imply

$$
\left|D\left(V^{(a)}-U^{(a)}\right)\right| \leqslant C \varepsilon
$$

in the region $\left\{|z| \leqslant \frac{3}{4} L,-\frac{3}{4} L \leqslant t \leqslant-\frac{1}{4} L\right\}$, and

$$
\left|D\left(V^{(a)}-U^{(a)}\right)\right| \leqslant C L^{2} \varepsilon
$$

in the region $\left\{|z| \leqslant \frac{3}{4} L,-\frac{1}{4} L \leqslant t \leqslant-1\right\}$. In particular, in $\left\{|z| \leqslant \frac{3}{4} L,-\frac{3}{4} L \leqslant t \leqslant-1\right\}$, the vector fields $V^{(1)}, V^{(2)}$, and $V^{(3)}$ are $C(L) \varepsilon_{1}$-close to the standard rotation vector fields on the cylinder in the $C^{1}$-norm. Consequently, in the region $\{|z| \leqslant 1000,-1000 \leqslant t \leqslant-1\}$, the vector fields $V^{(1)}, V^{(2)}$, and $V^{(3)}$ are $C(L) \varepsilon_{1}$-close to the standard rotation vector fields on the cylinder in the $C^{100}$-norm.

Step 3. We now define $h^{(a)}(t):=\mathscr{L}_{V^{(a)}(t)}(g(t))$. Since

$$
\frac{\partial}{\partial t} V^{(a)}=\Delta V^{(a)}+\operatorname{Ric}\left(V^{(a)}\right),
$$

we conclude that

$$
\frac{\partial}{\partial t} h^{(a)}(t)=\Delta_{L, g(t)} h^{(a)}(t)
$$


by Corollary 5.2. Using the estimate for $V^{(a)}-U^{(a)}$ in Step 2, we obtain

$$
\left|h^{(a)}\right| \leqslant\left|\mathscr{L}_{U^{(a)}}(g)\right|+C\left|D\left(V^{(a)}-U^{(a)}\right)\right| \leqslant C \varepsilon
$$

in the region $\left\{|z| \leqslant \frac{3}{4} L,-\frac{3}{4} L \leqslant t \leqslant-\frac{1}{4} L\right\}$, and

$$
\left|h^{(a)}\right| \leqslant\left|\mathscr{L}_{U^{(a)}}(g)\right|+C\left|D\left(V^{(a)}-U^{(a)}\right)\right| \leqslant C L^{2} \varepsilon
$$

in the region $\left\{|z| \leqslant \frac{3}{4} L,-\frac{1}{4} L \leqslant t \leqslant-1\right\}$. Using standard interior estimates for linear parabolic equations, we deduce that

$$
\sum_{l=0}^{100}\left|D^{l} h^{(a)}\right| \leqslant C(L) \varepsilon
$$

in the region $\left\{|z| \leqslant \frac{1}{2} L,-\frac{1}{2} L \leqslant t \leqslant-1\right\}$.

Step 4. By assumption,

$$
\sum_{l=0}^{100}\left|D^{l}(\bar{g}(t)-g(t))\right| \leqslant C(L) \varepsilon_{1}
$$

in the region $\left\{|z| \leqslant \frac{1}{2} L,-\frac{1}{2} L \leqslant t \leqslant-1\right\}$, where $\bar{g}(t)=(-2 t) g_{S^{2}}+d z \otimes d z$ denotes the standard metric on the shrinking cylinders. Let $\bar{h}^{(a)}$ denote the solution of the equation

$$
\frac{\partial}{\partial t} \bar{h}^{(a)}(t)=\Delta_{L, \bar{g}(t)} \bar{h}^{(a)}(t)
$$

in the region $\left\{|z| \leqslant \frac{1}{2} L,-\frac{1}{2} L \leqslant t \leqslant-1\right\}$ with Dirichlet boundary condition $\bar{h}^{(a)}=h^{(a)}$ on the parabolic boundary $\left\{|z| \leqslant \frac{1}{2} L, t=-\frac{1}{2} L\right\} \cup\left\{|z|=\frac{1}{2} L,-\frac{1}{2} L \leqslant t \leqslant-1\right\}$. We compute

$$
\frac{\partial}{\partial t}\left(\bar{h}^{(a)}(t)-h^{(a)}(t)\right)-\Delta_{L, \bar{g}(t)}\left(\bar{h}^{(a)}(t)-h^{(a)}(t)\right)=E^{(a)}(t),
$$

where the error term $E^{(a)}(t)$ is defined by

$$
E^{(a)}(t):=\Delta_{L, \bar{g}(t)} h^{(a)}(t)-\Delta_{L, g(t)} h^{(a)}(t)
$$

Using the estimates

$$
\sum_{l=0}^{100}\left|D^{l}(\bar{g}(t)-g(t))\right| \leqslant C(L) \varepsilon_{1} \quad \text { and } \quad \sum_{l=0}^{100}\left|D^{l} h^{(a)}\right| \leqslant C(L) \varepsilon
$$

we obtain

$$
\sum_{l=0}^{90}\left|D^{l} E^{(a)}\right| \leqslant C(L) \varepsilon_{1} \varepsilon
$$


in the region $\left\{|z| \leqslant \frac{1}{2} L,-\frac{1}{2} L \leqslant t \leqslant-1\right\}$. Using the maximum principle, we conclude that

$$
\left|\bar{h}^{(a)}-h^{(a)}\right| \leqslant C(L) \varepsilon_{1} \varepsilon
$$

in the region $\left\{|z| \leqslant \frac{1}{2} L,-\frac{1}{2} L \leqslant t \leqslant-1\right\}$. Standard interior estimates for linear parabolic equations imply

$$
\sum_{l=0}^{80}\left|D^{l}\left(\bar{h}^{(a)}-h^{(a)}\right)\right| \leqslant C(L) \varepsilon_{1} \varepsilon
$$

in the region $\{|z| \leqslant 1000,-1000 \leqslant t \leqslant-1\}$.

Step 5. We now apply Proposition 6.1 to $\bar{h}^{(a)}(t)$. Using the results in Steps 3 and 4 , we obtain

$$
\left|\bar{h}^{(a)}\right| \leqslant C \varepsilon+C(L) \varepsilon_{1} \varepsilon
$$

in the region $\left\{|z| \leqslant \frac{1}{2} L,-\frac{1}{2} L \leqslant t \leqslant-\frac{1}{4} L\right\}$, and

$$
\left|\bar{h}^{(a)}\right| \leqslant C L^{2} \varepsilon+C(L) \varepsilon_{1} \varepsilon
$$

in the region $\left\{|z| \leqslant \frac{1}{2} L,-\frac{1}{4} L \leqslant t \leqslant-1\right\}$. By Proposition 6.1, there is a function $\psi^{(a)}: S^{2} \rightarrow \mathbb{R}$ (independent of $z$ and $t$ ) and rotationally invariant functions $\bar{\omega}^{(a)}(z, t)$ and $\bar{\beta}^{(a)}(z, t)$ with the following properties:

- $\psi^{(a)}$ lies in the span of the first spherical harmonics on $S^{2}$;

- $\bar{\omega}^{(a)}(z, t)$ and $\bar{\beta}^{(a)}(z, t)$ are solutions of the 1-dimensional heat equation;

- $\left|\bar{h}^{(a)}(t)-\bar{\omega}^{(a)}(z, t) g_{S^{2}}-\bar{\beta}^{(a)}(z, t) d z \otimes d z-(-t) \psi^{(a)} g_{S^{2}}\right| \leqslant C L^{-1 / 2} \varepsilon+C(L) \varepsilon_{1} \varepsilon$ in the region $\{|z| \leqslant 1000,-1000 \leqslant t \leqslant-1\}$.

Note that $\bar{\omega}^{(a)}, \bar{\beta}^{(a)}$, and $\psi^{(a)}$ are bounded by $C(L) \varepsilon$. Moreover, the tensor

$$
\bar{h}^{(a)}(t)-\bar{\omega}^{(a)}(z, t) g_{S^{2}}-\bar{\beta}^{(a)}(z, t) d z \otimes d z-(-t) \psi^{(a)} g_{S^{2}}
$$

satisfies the parabolic Lichnerowicz equation with respect to the background metrics $\bar{g}(t)$. Using standard interior estimates for linear parabolic equations, we obtain

$$
\sum_{l=0}^{80}\left|D^{l}\left(\bar{h}^{(a)}(t)-\bar{\omega}^{(a)}(z, t) g_{S^{2}}-\bar{\beta}^{(a)}(z, t) d z \otimes d z-(-t) \psi^{(a)} g_{S^{2}}\right)\right| \leqslant C L^{-1 / 2} \varepsilon+C(L) \varepsilon_{1} \varepsilon
$$

in the region $\{|z| \leqslant 800,-400 \leqslant t \leqslant-1\}$. Combining this estimate with the estimate in Step 4, we conclude that

$$
\sum_{l=0}^{80}\left|D^{l}\left(h^{(a)}(t)-\bar{\omega}^{(a)}(z, t) g_{S^{2}}-\bar{\beta}^{(a)}(z, t) d z \otimes d z-(-t) \psi^{(a)} g_{S^{2}}\right)\right| \leqslant C L^{-1 / 2} \varepsilon+C(L) \varepsilon_{1} \varepsilon
$$

in the region $\{|z| \leqslant 800,-400 \leqslant t \leqslant-1\}$. 
Step 6. Let us define a vector field $\xi^{(a)}$ on $S^{2}$ by $g_{S^{2}}\left(\xi^{(a)}, \cdot\right)=-\frac{1}{4} d \psi^{(a)}$. Note that $\xi^{(a)}$ is independent of $z$ and $t$, and $\left|\xi^{(a)}\right| \leqslant C(L) \varepsilon$. Since $\psi^{(a)}$ lies in the span of the first spherical harmonic on $S^{2}$, we obtain $\mathscr{L}_{\xi^{(a)}}\left(g_{S^{2}}\right)=\frac{1}{2} \psi^{(a)} g_{S^{2}}$, and hence

$$
\mathscr{L}_{\xi^{(a)}}(\bar{g}(t))=(-t) \psi^{(a)} g_{S^{2}}
$$

We now define $W^{(a)}:=V^{(a)}-\xi^{(a)}$. In the region $\{|z| \leqslant 1000,-1000 \leqslant t \leqslant-1\}$, the vector fields $W^{(1)}, W^{(2)}$, and $W^{(3)}$ are $C(L) \varepsilon_{1}$-close to the standard rotation vector fields on the cylinder in the $C^{80}$-norm. Using the identity

$$
\mathscr{L}_{W^{(a)}}(g(t))=h^{(a)}(t)-(-t) \psi^{(a)} g_{S^{2}}+\mathscr{L}_{\xi^{(a)}}(\bar{g}(t)-g(t))
$$

and the estimates in Step 5, we obtain

$$
\sum_{l=0}^{60}\left|D^{l}\left(\mathscr{L}_{W^{(a)}}(g(t))-\bar{\omega}^{(a)}(z, t) g_{S^{2}}-\bar{\beta}^{(a)}(z, t) d z \otimes d z\right)\right| \leqslant C L^{-1 / 2} \varepsilon+C(L) \varepsilon_{1} \varepsilon
$$

in the region $\{|z| \leqslant 800,-400 \leqslant t \leqslant-1\}$.

We next estimate the time derivative of $W^{(a)}$. We compute

$$
\frac{\partial}{\partial t} W^{(a)}=\frac{\partial}{\partial t} V^{(a)}=\Delta V^{(a)}+\operatorname{Ric}\left(V^{(a)}\right)=\operatorname{div} h^{(a)}-\frac{1}{2} \nabla\left(\operatorname{tr} h^{(a)}\right) .
$$

Using the estimates in Step 5, we obtain

$$
\sum_{l=0}^{60}\left|D^{l}\left(\operatorname{div} h^{(a)}-\frac{\partial \bar{\beta}^{(a)}}{\partial z} \frac{\partial}{\partial z}-\frac{1}{-t} \xi^{(a)}\right)\right| \leqslant C L^{-1 / 2} \varepsilon+C(L) \varepsilon_{1} \varepsilon
$$

and

$$
\sum_{l=0}^{60}\left|D^{l}\left(\nabla\left(\operatorname{tr} h^{(a)}\right)-\left(\frac{\partial \bar{\beta}^{(a)}}{\partial z}+\frac{1}{-t} \frac{\partial \bar{\omega}^{(a)}}{\partial z}\right) \frac{\partial}{\partial z}-\frac{2}{-t} \xi^{(a)}\right)\right| \leqslant C L^{-1 / 2} \varepsilon+C(L) \varepsilon_{1} \varepsilon
$$

in the region $\{|z| \leqslant 800,-400 \leqslant t \leqslant-1\}$. Putting these facts together, we conclude that

$$
\sum_{l=0}^{60}\left|D^{l}\left(\frac{\partial}{\partial t} W^{(a)}-\left(\frac{1}{2} \frac{\partial \bar{\beta}^{(a)}}{\partial z}-\frac{1}{-2 t} \frac{\partial \bar{\omega}^{(a)}}{\partial z}\right) \frac{\partial}{\partial z}\right)\right| \leqslant C L^{-1 / 2} \varepsilon+C(L) \varepsilon_{1} \varepsilon
$$

in the region $\{|z| \leqslant 800,-400 \leqslant t \leqslant-1\}$.

Step 7. We now define

$$
X^{(1)}:=\left[W^{(2)}, W^{(3)}\right], \quad X^{(2)}:=\left[W^{(3)}, W^{(1)}\right], \quad \text { and } \quad X^{(3)}:=\left[W^{(1)}, W^{(2)}\right] .
$$


In the region $\{|z| \leqslant 800,-400 \leqslant t \leqslant-1\}$, the vector fields $X^{(1)}, X^{(2)}$, and $X^{(3)}$ agree with the standard rotation vector fields on the cylinder up to constant factors and errors of order $C(L) \varepsilon_{1}$. Moreover,

$$
\begin{aligned}
\mathscr{L}_{X^{(1)}}(g)= & \mathscr{L}_{W^{(2)}}\left(\mathscr{L}_{W^{(3)}}(g)\right)-\mathscr{L}_{W^{(3)}}\left(\mathscr{L}_{W^{(2)}}(g)\right) \\
= & \mathscr{L}_{W^{(2)}}\left(\mathscr{L}_{W^{(3)}}(g)-\bar{\omega}^{(3)}(z, t) g_{S^{2}}-\bar{\beta}^{(3)}(z, t) d z \otimes d z\right) \\
& -\mathscr{L}_{W^{(3)}}\left(\mathscr{L}_{W^{(2)}}(g)-\bar{\omega}^{(2)}(z, t) g_{S^{2}}-\bar{\beta}^{(2)}(z, t) d z \otimes d z\right) \\
& +\mathscr{L}_{W^{(2)}}\left(\bar{\omega}^{(3)}(z, t) g_{S^{2}}+\bar{\beta}^{(3)}(z, t) d z \otimes d z\right) \\
& -\mathscr{L}_{W^{(3)}}\left(\bar{\omega}^{(2)}(z, t) g_{S^{2}}+\bar{\beta}^{(2)}(z, t) d z \otimes d z\right) .
\end{aligned}
$$

Analogous identities hold for $\mathscr{L}_{X^{(2)}}(g)$ and $\mathscr{L}_{X^{(3)}}(g)$. Since $W^{(1)}, W^{(2)}$, and $W^{(3)}$ agree with the standard rotation vector fields on the cylinder up to errors of order $C(L) \varepsilon_{1}$, we obtain

$$
\sum_{l=0}^{40}\left|D^{l}\left(\mathscr{L}_{W^{(a)}}\left(\bar{\omega}^{(b)}(z, t) g_{S^{2}}+\bar{\beta}^{(b)}(z, t) d z \otimes d z\right)\right)\right| \leqslant C(L) \varepsilon_{1} \varepsilon
$$

in the region $\{|z| \leqslant 800,-400 \leqslant t \leqslant-1\}$. Combining this with the estimates in Step 6, we conclude that

$$
\sum_{l=0}^{40}\left|D^{l}\left(\mathscr{L}_{X^{(a)}}(g)\right)\right| \leqslant C L^{-1 / 2} \varepsilon+C(L) \varepsilon_{1} \varepsilon
$$

in the region $\{|z| \leqslant 800,-400 \leqslant t \leqslant-1\}$.

We now estimate the time derivative of $X^{(a)}$. We compute

$$
\begin{aligned}
\frac{\partial}{\partial t} X^{(1)}= & {\left[\frac{\partial}{\partial t} W^{(2)}, W^{(3)}\right]+\left[W^{(2)}, \frac{\partial}{\partial t} W^{(3)}\right] } \\
=[ & \left.\frac{\partial}{\partial t} W^{(2)}-\left(\frac{1}{2} \frac{\partial \bar{\beta}^{(2)}}{\partial z}-\frac{1}{-2 t} \frac{\partial \bar{\omega}^{(2)}}{\partial z}\right) \frac{\partial}{\partial z}, W^{(3)}\right] \\
& +\left[W^{(2)}, \frac{\partial}{\partial t} W^{(3)}-\left(\frac{1}{2} \frac{\partial \bar{\beta}^{(3)}}{\partial z}-\frac{1}{-2 t} \frac{\partial \bar{\omega}^{(3)}}{\partial z}\right) \frac{\partial}{\partial z}\right] \\
+ & {\left[\left(\frac{1}{2} \frac{\partial \bar{\beta}^{(2)}}{\partial z}-\frac{1}{-2 t} \frac{\partial \bar{\omega}^{(2)}}{\partial z}\right) \frac{\partial}{\partial z}, W^{(3)}\right] } \\
& +\left[W^{(2)},\left(\frac{1}{2} \frac{\partial \bar{\beta}^{(3)}}{\partial z}-\frac{1}{-2 t} \frac{\partial \bar{\omega}^{(3)}}{\partial z}\right) \frac{\partial}{\partial z}\right] .
\end{aligned}
$$

Analogous identities hold for $\partial X^{(2)} / \partial t$ and $\partial X^{(3)} / \partial t$. Since $W^{(1)}, W^{(2)}$, and $W^{(3)}$ agree with the standard rotation vector fields on the cylinder up to errors of order $C(L) \varepsilon_{1}$, we obtain

$$
\sum_{l=0}^{40}\left|D^{l}\left[W^{(a)},\left(\frac{1}{2} \frac{\partial \bar{\beta}^{(b)}}{\partial z}-\frac{1}{-2 t} \frac{\partial \bar{\omega}^{(b)}}{\partial z}\right) \frac{\partial}{\partial z}\right]\right| \leqslant C(L) \varepsilon_{1} \varepsilon
$$


in the region $\{|z| \leqslant 800,-400 \leqslant t \leqslant-1\}$. Combining this with the estimates in Step 6, we conclude that

$$
\sum_{l=0}^{40}\left|D^{l}\left(\frac{\partial}{\partial t} X^{(a)}\right)\right| \leqslant C L^{-1 / 2} \varepsilon+C(L) \varepsilon_{1} \varepsilon
$$

in the region $\{|z| \leqslant 800,-400 \leqslant t \leqslant-1\}$.

Step 8. Let $Y^{(a)}$ be a time-independent vector field such that $Y^{(a)}=X^{(a)}$ at time -1 . In the region $\{|z| \leqslant 800,-400 \leqslant t \leqslant-1\}$, the vector fields $Y^{(1)}, Y^{(2)}$, and $Y^{(3)}$ agree with the standard rotation vector fields on the cylinder up to constant factors and errors of order $C(L) \varepsilon_{1}$. The estimates for $\partial X^{(a)} / \partial t$ in Step 7 imply

$$
\sum_{l=0}^{40}\left|D^{l}\left(Y^{(a)}-X^{(a)}\right)\right| \leqslant C L^{-1 / 2} \varepsilon+C(L) \varepsilon_{1} \varepsilon
$$

in the region $\{|z| \leqslant 800,-400 \leqslant t \leqslant-1\}$. Using the estimates for $\mathscr{L}_{X^{(a)}}(g)$ in Step 6 , we obtain

$$
\sum_{l=0}^{30}\left|D^{l}\left(\mathscr{L}_{Y^{(a)}}(g)\right)\right| \leqslant C L^{-1 / 2} \varepsilon+C(L) \varepsilon_{1} \varepsilon
$$

in the region $\{|z| \leqslant 800,-400 \leqslant t \leqslant-1\}$.

Step 9. In the following, we fix a time $t \in[-200,-1]$. We denote by $\Sigma_{s}$ the leaves of the CMC foliation of $(M, g(t))$. Note that the foliation depends on $t$, but we suppress this dependence in the notation. Let $\nu$ denote the unit normal vector field to the foliation $\Sigma_{s}$. For each $s$, we denote by $v: \Sigma_{s} \rightarrow \mathbb{R}$ the lapse function associated with this foliation. We assume that the foliation $\Sigma_{s}$ is parameterized so that $x_{0} \in \Sigma_{0}$ and $\int_{\Sigma_{s}} v=1$ for all $s$. Since $\Sigma_{s}$ is a CMC surface for each $s$, the function $v$ satisfies the Jacobi equation

$$
\Delta_{\Sigma_{s}} v+\left(|A|^{2}+\operatorname{Ric}(\nu, \nu)\right) v=\text { constant }
$$

on $\Sigma_{s}$, where $|A|$ denotes the norm of the second fundamental form of $\Sigma_{s}$ in $(M, g(t))$. The Jacobi operator $\Delta_{\Sigma_{s}}+\left(|A|^{2}+\operatorname{Ric}(\nu, \nu)\right)$ is a small perturbation of the Laplacian $\Delta_{\Sigma_{s}}$. Hence, for each $s$, the Jacobi operator $\Delta_{\Sigma_{s}}+\left(|A|^{2}+\operatorname{Ric}(\nu, \nu)\right)$ is an invertible operator from the space $\left\{f \in C^{2,1 / 2}\left(\Sigma_{s}\right): \int_{\Sigma_{s}} f=0\right\}$ to the space $\left\{f \in C^{1 / 2}\left(\Sigma_{s}\right): \int_{\Sigma_{s}} f v=0\right\}$, and we have a uniform bound for the norm of its inverse.

In the following, we only consider those leaves of the foliation $\Sigma_{s}$ which are contained in the region $\{|z| \leqslant 700\}$. Let us define a function $F^{(a)}: \Sigma_{s} \rightarrow \mathbb{R}$ by $F^{(a)}:=\left\langle Y^{(a)}, \nu\right\rangle$. The quantity

$$
\Delta_{\Sigma_{s}} F^{(a)}+\left(|A|^{2}+\operatorname{Ric}(\nu, \nu)\right) F^{(a)}=: H^{(a)}
$$


can be expressed in terms of $\mathscr{L}_{Y^{(a)}}(g)$ and the first derivatives of $\mathscr{L}_{Y^{(a)}}(g)$. Using the estimate for $\mathscr{L}_{Y^{(a)}}(g)$ in Step 8, we deduce that

$$
\sum_{l=0}^{20}\left|D^{l} H^{(a)}\right| \leqslant C L^{-1 / 2} \varepsilon+C(L) \varepsilon_{1} \varepsilon
$$

in the region $\{|z| \leqslant 700\}$. We next define $G^{(a)}(s):=\int_{\Sigma_{s}} F^{(a)}$ and $\widetilde{F}^{(a)}:=F^{(a)}-G^{(a)}(s) v$. Then $\int_{\Sigma_{s}} \widetilde{F}^{(a)}=0$ and

$$
\Delta_{\Sigma_{s}} \widetilde{F}^{(a)}+\left(|A|^{2}+\operatorname{Ric}(\nu, \nu)\right) \widetilde{F}^{(a)}=H^{(a)}-\int_{\Sigma_{s}} H^{(a)} v
$$

on $\Sigma_{s}$. Using the estimate

$$
\sum_{l=0}^{20}\left|D^{l} H^{(a)}\right| \leqslant C L^{-1 / 2} \varepsilon+C(L) \varepsilon_{1} \varepsilon,
$$

we conclude that

$$
\sum_{l=0}^{10}\left|D^{l} \widetilde{F}^{(a)}\right| \leqslant C L^{-1 / 2} \varepsilon+C(L) \varepsilon_{1} \varepsilon
$$

in the region $\{|z| \leqslant 600\}$. Since $v^{-1} \widetilde{F}^{(a)}=v^{-1}\left\langle Y^{(a)}, \nu\right\rangle-G^{(a)}(s)$, it follows that

$$
\sum_{l=0}^{10}\left|D^{l}\left(v^{-1}\left\langle Y^{(a)}, \nu\right\rangle-G^{(a)}(s)\right)\right| \leqslant C L^{-1 / 2} \varepsilon+C(L) \varepsilon_{1} \varepsilon
$$

in the region $\{|z| \leqslant 600\}$.

By the divergence theorem, the quantity

$$
G^{(a)}(s)-G^{(a)}(0)=\int_{\Sigma_{s}}\left\langle Y^{(a)}, \nu\right\rangle-\int_{\Sigma_{0}}\left\langle Y^{(a)}, \nu\right\rangle
$$

can be expressed as an integral of $\operatorname{div} Y^{(a)}$ over the region bounded by $\Sigma_{0}$ and $\Sigma_{s}$. Differentiating this identity with respect to $s$ gives

$$
\frac{d}{d s} G^{(a)}(s)=\int_{\Sigma_{s}} v \operatorname{div} Y^{(a)} .
$$

Using the estimate for $\mathscr{L}_{Y^{(a)}}(g)$ in Step 8, we obtain

$$
\sum_{l=1}^{10}\left|\frac{d^{l}}{d s^{l}} G^{(a)}(s)\right| \leqslant C L^{-1 / 2} \varepsilon+C(L) \varepsilon_{1} \varepsilon .
$$


Putting these facts together, we conclude that

$$
\sum_{l=1}^{10}\left|D^{l}\left(v^{-1}\left\langle Y^{(a)}, \nu\right\rangle\right)\right| \leqslant C L^{-1 / 2} \varepsilon+C(L) \varepsilon_{1} \varepsilon
$$

in the region $\{|z| \leqslant 600\}$.

Step 10. Finally, we define

$$
Z^{(1)}:=\left[Y^{(2)}, Y^{(3)}\right], \quad Z^{(2)}:=\left[Y^{(3)}, Y^{(1)}\right] \quad \text { and } \quad Z^{(3)}:=\left[Y^{(1)}, Y^{(2)}\right]
$$

We note that $Z^{(1)}, Z^{(2)}$, and $Z^{(3)}$ are time-independent vector fields. In the region $\{|z| \leqslant 800,-400 \leqslant t \leqslant-1\}$, the vector fields $Z^{(1)}, Z^{(2)}$, and $Z^{(3)}$ agree with the standard rotation vector fields on the cylinder up to constant factors and errors of order $C(L) \varepsilon_{1}$. Note that

$$
\mathscr{L}_{Z^{(1)}}(g)=\mathscr{L}_{Y^{(2)}}\left(\mathscr{L}_{Y^{(3)}}(g)\right)-\mathscr{L}_{Y^{(3)}}\left(\mathscr{L}_{Y^{(2)}}(g)\right)
$$

We have shown in Step 8 that

$$
\sum_{l=0}^{30}\left|D^{l}\left(\mathscr{L}_{Y^{(a)}}(g)\right)\right| \leqslant C L^{-1 / 2} \varepsilon+C(L) \varepsilon_{1} \varepsilon
$$

in the region $\{|z| \leqslant 500, t \in[-200,-1]\}$. This gives

$$
\sum_{l=0}^{20}\left|D^{l}\left(\mathscr{L}_{Z^{(a)}}(g)\right)\right| \leqslant C L^{-1 / 2} \varepsilon+C(L) \varepsilon_{1} \varepsilon
$$

in the region $\{|z| \leqslant 500,-200 \leqslant t \leqslant-1\}$. Now, let us fix a time $t \in[-200,-1]$, and let $\nu$ and $v$ denote the normal vector field and the lapse function of the CMC foliation at time $t$, respectively. Since the vector field $T:=v^{-1} \nu$ is a gradient vector field, we have

$$
\left\langle Z^{(1)}, T\right\rangle=\left\langle Y^{(2)}, \nabla\left(\left\langle Y^{(3)}, T\right\rangle\right)\right\rangle-\left\langle Y^{(3)}, \nabla\left(\left\langle Y^{(2)}, T\right\rangle\right)\right\rangle .
$$

Using the estimates in Step 9, we obtain

$$
\sum_{l=1}^{10}\left|D^{l}\left(\left\langle Y^{(a)}, T\right\rangle\right)\right| \leqslant C L^{-1 / 2} \varepsilon+C(L) \varepsilon_{1} \varepsilon
$$

in the region $\{|z| \leqslant 500,-200 \leqslant t \leqslant-1\}$. Consequently,

$$
\sum_{l=0}^{8}\left|D^{l}\left(\left\langle Z^{(a)}, T\right\rangle\right)\right| \leqslant C L^{-1 / 2} \varepsilon+C(L) \varepsilon_{1} \varepsilon
$$


in the region $\{|z| \leqslant 500,-200 \leqslant t \leqslant-1\}$. Using again the fact that $T$ is a gradient vector field, we compute

$$
\left\langle\nabla\left(|T|^{2}\right), Z^{(a)}\right\rangle=-\left(\mathscr{L}_{Z^{(a)}}(g)\right)(T, T)+2\left\langle\nabla\left(\left\langle Z^{(a)}, T\right\rangle\right), T\right\rangle
$$

and

$$
g\left(\left[T, Z^{(a)}\right], \cdot\right)=\left(\mathscr{L}_{Z^{(a)}}(g)\right)(T, \cdot)-d\left(\left\langle Z^{(a)}, T\right\rangle\right) .
$$

Using our estimates for $\mathscr{L}_{Z^{(a)}}(g)$ and $\left\langle Z^{(a)}, T\right\rangle$, we finally obtain

$$
\sum_{l=0}^{6}\left|D^{l}\left(\left\langle\nabla\left(|T|^{2}\right), Z^{(a)}\right\rangle\right)\right| \leqslant C L^{-1 / 2} \varepsilon+C(L) \varepsilon_{1} \varepsilon
$$

and

$$
\sum_{l=0}^{6}\left|D^{l}\left(\left[T, Z^{(a)}\right]\right)\right| \leqslant C L^{-1 / 2} \varepsilon+C(L) \varepsilon_{1} \varepsilon
$$

in the region $\{|z| \leqslant 500,-200 \leqslant t \leqslant-1\}$.

To summarize, we have shown that

$$
\begin{gathered}
\sum_{l=0}^{8}\left|D^{l}\left(\left\langle Z^{(a)}, \nu\right\rangle\right)\right| \leqslant C L^{-1 / 2} \varepsilon+C(L) \varepsilon_{1} \varepsilon \\
\sum_{l=0}^{6}\left|D^{l}\left(\left\langle\nabla v, Z^{(a)}\right\rangle\right)\right| \leqslant C L^{-1 / 2} \varepsilon+C(L) \varepsilon_{1} \varepsilon \\
\sum_{l=0}^{6}\left|D^{l}\left(\left[\nu, Z^{(a)}\right]\right)\right| \leqslant C L^{-1 / 2} \varepsilon+C(L) \varepsilon_{1} \varepsilon \\
\sum_{l=0}^{6}\left|D^{l}\left(\left[v \nu, Z^{(a)}\right]\right)\right| \leqslant C L^{-1 / 2} \varepsilon+C(L) \varepsilon_{1} \varepsilon
\end{gathered}
$$

in the region $\{|z| \leqslant 500,-200 \leqslant t \leqslant-1\}$. In particular, if $t \in[-200,-1]$ and $\Sigma \subset\{|z| \leqslant 400\}$ is a leaf of the CMC foliation in $(M, g(t))$, then the lapse function $v$ satisfies

$$
\sup _{\Sigma}\left|v-\operatorname{area}_{g(t)}(\Sigma)^{-1}\right| \leqslant C L^{-1 / 2} \varepsilon+C(L) \varepsilon_{1} \varepsilon
$$

Step 11. In the next step, we obtain information on the Ricci tensor and the second fundamental form of the CMC foliation. To that end, let us consider an arbitrary point $(\bar{x}, \bar{t})$ in the region $\{|z| \leqslant 400,-200 \leqslant t \leqslant-1\}$. Let $\left\{e_{1}, e_{2}\right\}$ denote an orthonormal basis for the tangent space to the CMC foliation at $(\bar{x}, \bar{t})$. Since the vector fields $Z^{(1)}, Z^{(2)}$, and 
$Z^{(3)}$ are close to the standard rotation vector fields on the cylinder up to some constant factor, we can find a vector $\lambda=\left(\lambda_{1}, \lambda_{2}, \lambda_{3}\right) \in \mathbb{R}^{3}$ such that

$$
\sum_{a=1}^{3} \lambda_{a}\left\langle Z^{(a)}, e_{1}\right\rangle=\sum_{a=1}^{3} \lambda_{a}\left\langle Z^{(a)}, e_{2}\right\rangle=0
$$

at the point $(\bar{x}, \bar{t})$ and

$$
\sum_{a=1}^{3} \lambda_{a}\left\langle D_{e_{1}} Z^{(a)}, e_{2}\right\rangle=1
$$

at the point $(\bar{x}, \bar{t})$. Note that $|\lambda| \leqslant C$.

Using the estimate for $\left\langle Z^{(a)}, \nu\right\rangle$ in Step 10, we obtain

$$
\left|\sum_{a=1}^{3} \lambda_{a}\left\langle Z^{(a)}, \nu\right\rangle\right| \leqslant C L^{-1 / 2} \varepsilon+C(L) \varepsilon_{1} \varepsilon .
$$

Consequently,

$$
\left|\sum_{a=1}^{3} \lambda_{a} Z^{(a)}\right| \leqslant C L^{-1 / 2} \varepsilon+C(L) \varepsilon_{1} \varepsilon
$$

at the point $(\bar{x}, \bar{t})$. Using the estimate for $\mathscr{L}_{Z^{(a)}}(g)$ in Step 10 , we obtain

$$
\begin{gathered}
\left|\left\langle D_{e_{1}} Z^{(a)}, e_{1}\right\rangle\right|+\left|\left\langle D_{e_{2}} Z^{(a)}, e_{2}\right\rangle\right| \leqslant C L^{-1 / 2} \varepsilon+C(L) \varepsilon_{1} \varepsilon \\
\left|\left\langle D_{e_{1}} Z^{(a)}, e_{2}\right\rangle+\left\langle D_{e_{2}} Z^{(a)}, e_{1}\right\rangle\right| \leqslant C L^{-1 / 2} \varepsilon+C(L) \varepsilon_{1} \varepsilon .
\end{gathered}
$$

This implies

$$
\left|1+\sum_{a=1}^{3} \lambda_{a}\left\langle D_{e_{2}} Z^{(a)}, e_{1}\right\rangle\right| \leqslant C L^{-1 / 2} \varepsilon+C(L) \varepsilon_{1} \varepsilon
$$

at the point $(\bar{x}, \bar{t})$. Moreover, the estimate for the derivatives of $\left\langle Z^{(a)}, \nu\right\rangle$ in Step 10 gives

$$
\left|\left\langle D_{e_{i}} Z^{(a)}, \nu\right\rangle+\left\langle Z^{(a)}, D_{e_{i}} \nu\right\rangle\right| \leqslant C L^{-1 / 2} \varepsilon+C(L) \varepsilon_{1} \varepsilon
$$

for $i \in\{1,2\}$. Hence, for each $i \in\{1,2\}$, we obtain

$$
\left|\sum_{a=1}^{3} \lambda_{a}\left\langle D_{e_{i}} Z^{(a)}, \nu\right\rangle\right| \leqslant C L^{-1 / 2} \varepsilon+C(L) \varepsilon_{1} \varepsilon
$$

at the point $(\bar{x}, \bar{t})$.

In view of the estimates in Step 10, the Ricci tensor satisfies

$$
\left|\mathscr{L}_{Z^{(a)}} \operatorname{Ric}\right| \leqslant C L^{-1 / 2} \varepsilon+C(L) \varepsilon_{1} \varepsilon
$$


for each $a \in\{1,2,3\}$. A straightforward calculation gives

$$
\begin{aligned}
\left(\mathscr{L}_{Z^{(a)}} \operatorname{Ric}\right)\left(e_{1}, e_{2}\right)=( & \left.D_{Z^{(a)}} \operatorname{Ric}\right)\left(e_{1}, e_{2}\right)+\operatorname{Ric}\left(D_{e_{1}} Z^{(a)}, e_{2}\right)+\operatorname{Ric}\left(e_{1}, D_{e_{2}} Z^{(a)}\right) \\
= & \left(D_{Z^{(a)}} \operatorname{Ric}\right)\left(e_{1}, e_{2}\right)+\left(\left\langle D_{e_{1}} Z^{(a)}, e_{1}\right\rangle+\left\langle D_{e_{2}} Z^{(a)}, e_{2}\right\rangle\right) \operatorname{Ric}\left(e_{1}, e_{2}\right) \\
& +\left\langle D_{e_{1}} Z^{(a)}, e_{2}\right\rangle \operatorname{Ric}\left(e_{2}, e_{2}\right)+\left\langle D_{e_{2}} Z^{(a)}, e_{1}\right\rangle \operatorname{Ric}\left(e_{1}, e_{1}\right) \\
& +\left\langle D_{e_{1}} Z^{(a)}, \nu\right\rangle \operatorname{Ric}\left(\nu, e_{2}\right)+\left\langle D_{e_{2}} Z^{(a)}, \nu\right\rangle \operatorname{Ric}\left(e_{1}, \nu\right) .
\end{aligned}
$$

If we multiply this identity by $\lambda_{a}$ and sum over $a \in\{1,2,3\}$, we conclude that

$$
\left|\operatorname{Ric}\left(e_{2}, e_{2}\right)-\operatorname{Ric}\left(e_{1}, e_{1}\right)\right| \leqslant C L^{-1 / 2} \varepsilon+C(L) \varepsilon_{1} \varepsilon
$$

at the point $(\bar{x}, \bar{t})$. Therefore,

$$
\left|\operatorname{Ric}\left(e_{i}, e_{j}\right)-\frac{1}{2} \operatorname{tr}_{\Sigma}(\operatorname{Ric}) \delta_{i j}\right| \leqslant C L^{-1 / 2} \varepsilon+C(L) \varepsilon_{1} \varepsilon
$$

at the point $(\bar{x}, \bar{t})$, where

$$
\operatorname{tr}_{\Sigma}(\operatorname{Ric})=\operatorname{Ric}\left(e_{1}, e_{1}\right)+\operatorname{Ric}\left(e_{2}, e_{2}\right) .
$$

Let $A$ denote the second fundamental form of the CMC foliation. We can think of $A$ as a $(0,2)$-tensor on $M$, which vanishes in the normal direction. The estimates in Step 10 imply $\left|\mathscr{L}_{Z^{(a)}} A\right| \leqslant C L^{-1 / 2} \varepsilon+C(L) \varepsilon_{1} \varepsilon$ for each $a \in\{1,2,3\}$. A straightforward calculation gives

$$
\begin{aligned}
\left(\mathscr{L}_{Z^{(a)}} A\right)\left(e_{1}, e_{2}\right)= & \left(D_{Z^{(a)}} A\right)\left(e_{1}, e_{2}\right)+A\left(D_{e_{1}} Z^{(a)}, e_{2}\right)+A\left(e_{1}, D_{e_{2}} Z^{(a)}\right) \\
= & \left(D_{Z^{(a)}} A\right)\left(e_{1}, e_{2}\right)+\left(\left\langle D_{e_{1}} Z^{(a)}, e_{1}\right\rangle+\left\langle D_{e_{2}} Z^{(a)}, e_{2}\right\rangle\right) A\left(e_{1}, e_{2}\right) \\
& +\left\langle D_{e_{1}} Z^{(a)}, e_{2}\right\rangle A\left(e_{2}, e_{2}\right)+\left\langle D_{e_{2}} Z^{(a)}, e_{1}\right\rangle A\left(e_{1}, e_{1}\right) .
\end{aligned}
$$

If we multiply this identity by $\lambda_{a}$ and sum over $a \in\{1,2,3\}$, we conclude that

$$
\left|A\left(e_{2}, e_{2}\right)-A\left(e_{1}, e_{1}\right)\right| \leqslant C L^{-1 / 2} \varepsilon+C(L) \varepsilon_{1} \varepsilon
$$

at the point $(\bar{x}, \bar{t})$. Therefore,

$$
\left|A\left(e_{i}, e_{j}\right)-\frac{1}{2} H \delta_{i j}\right| \leqslant C L^{-1 / 2} \varepsilon+C(L) \varepsilon_{1} \varepsilon
$$

at the point $(\bar{x}, \bar{t})$, where $H$ denotes the mean curvature of the CMC foliation.

Finally, the estimates in Step 10 imply that

$$
\inf _{\varrho} \sup _{\Sigma}\left|\frac{1}{2} \operatorname{tr}_{\Sigma}(\mathrm{Ric})-\varrho\right| \leqslant C L^{-1 / 2} \varepsilon+C(L) \varepsilon_{1} \varepsilon
$$


if $t \in[-200,-1]$ and $\Sigma \subset\{|z| \leqslant 400\}$ is a leaf of the CMC foliation in $(M, g(t))$. To summarize, if $t \in[-200,-1]$ and $\Sigma \subset\{|z| \leqslant 400\}$ is a leaf of the CMC foliation in $(M, g(t))$, then

$$
\inf _{\varrho} \sup _{\Sigma}|(\operatorname{Ric}-\varrho g)|_{T \Sigma} \mid \leqslant C L^{-1 / 2} \varepsilon+C(L) \varepsilon_{1} \varepsilon
$$

and

$$
\sup _{\Sigma}\left|\left(A-\frac{1}{2} H g\right)_{T \Sigma}\right| \leqslant C L^{-1 / 2} \varepsilon+C(L) \varepsilon_{1} \varepsilon
$$

where $H$ denotes the mean curvature of $\Sigma$ (which is constant).

Step 12. Let us fix a time $t \in[-200,-1]$. By Step 10, the vector fields $Z^{(1)}, Z^{(2)}$, and $Z^{(3)}$ are tangential to the CMC foliation of $(M, g(t))$, up to errors of order

$$
C L^{-1 / 2} \varepsilon+C(L) \varepsilon_{1} \varepsilon
$$

Moreover, the vector fields $Z^{(1)}, Z^{(2)}$, and $Z^{(3)}$ are tangential to the CMC foliation of $(M, g(-1))$, up to errors of order $C L^{-1 / 2} \varepsilon+C(L) \varepsilon_{1} \varepsilon$. Since the vector fields $Z^{(1)}, Z^{(2)}$, and $Z^{(3)}$ are close to the standard rotation vector fields on the cylinder, we conclude that every leaf of the CMC foliation of $(M, g(t))$ which is contained in the region $\{|z| \leqslant 400\}$ is $\left(C L^{-1 / 2}+C(L) \varepsilon_{1} \varepsilon\right)$-close in the $C^{1}$-norm to a leaf of the CMC foliation of $(M, g(-1))$.

Step 13. We again fix a time $t \in[-200,-1]$. Let $\Sigma_{s}$ denote the CMC foliation of $(M, g(t))$, and let $\nu$ and $v$ denote the normal vector field and the lapse function associated with this foliation, respectively. In the following, we only consider those leaves of the foliation which are contained in the region $\{|z| \leqslant 300\}$. Our goal is to show that the quantity

$$
\operatorname{area}_{g(t)}\left(\Sigma_{s}\right)^{-2} \int_{\Sigma_{s}}\left\langle Z^{(a)}, Z^{(b)}\right\rangle_{g(t)} d \mu_{g(t)}
$$

is nearly constant in $s$, up to errors of order $C L^{-1 / 2} \varepsilon+C(L) \varepsilon_{1} \varepsilon$. Recall that the surfaces $\Sigma_{s}$ move with normal velocity $v$. This implies that

$$
\frac{d}{d s} \text { area }_{g(t)}\left(\Sigma_{s}\right)=\int_{\Sigma_{s}} H v d \mu_{g(t)}=H,
$$

where $H$ denotes the mean curvature of $\Sigma_{s}$ with respect to the metric $g(t)$. We next compute

$$
\begin{aligned}
& \frac{d}{d s}\left(\int_{\Sigma_{s}}\left\langle Z^{(a)}, Z^{(b)}\right\rangle_{g(t)} d \mu_{g(t)}\right) \\
& \quad=\int_{\Sigma_{s}} H v\left\langle Z^{(a)}, Z^{(b)}\right\rangle_{g(t)} d \mu_{g(t)}+\int_{\Sigma_{s}}\left(\mathscr{L}_{v \nu}(g)\right)\left(Z^{(a)}, Z^{(b)}\right) d \mu_{g(t)} \\
& \quad+\int_{\Sigma_{s}}\left\langle\left[v \nu, Z^{(a)}\right], Z^{(b)}\right\rangle_{g(t)} d \mu_{g(t)}+\int_{\Sigma_{s}}\left\langle Z^{(a)},\left[v \nu, Z^{(b)}\right]\right\rangle_{g(t)} d \mu_{g(t)}
\end{aligned}
$$


The estimate $\left|\left(A-\frac{1}{2} H g\right)\right|_{T \Sigma} \mid \leqslant C L^{-1 / 2} \varepsilon+C(L) \varepsilon_{1} \varepsilon$ in Step 11 implies that

$$
\left|\left(\mathscr{L}_{v \nu}(g)\right)\left(Z^{(a)}, Z^{(b)}\right)-H v\left\langle Z^{(a)}, Z^{(b)}\right\rangle_{g(t)}\right| \leqslant C L^{-1 / 2} \varepsilon+C(L) \varepsilon_{1} \varepsilon .
$$

Moreover, the estimate for $\left[v \nu, Z^{(a)}\right]$ in Step 10 gives

$$
\left|\left\langle\left[v \nu, Z^{(a)}\right], Z^{(b)}\right\rangle_{g(t)}\right| \leqslant C L^{-1 / 2} \varepsilon+C(L) \varepsilon_{1} \varepsilon .
$$

An analogous argument yields

$$
\left|\left\langle Z^{(a)},\left[v \nu, Z^{(b)}\right]\right\rangle_{g(t)}\right| \leqslant C L^{-1 / 2} \varepsilon+C(L) \varepsilon_{1} \varepsilon .
$$

Putting these facts together, we obtain

$\left|\frac{d}{d s}\left(\int_{\Sigma_{s}}\left\langle Z^{(a)}, Z^{(b)}\right\rangle_{g(t)} d \mu_{g(t)}\right)-2 \int_{\Sigma_{s}} H v\left\langle Z^{(a)}, Z^{(b)}\right\rangle_{g(t)} d \mu_{g(t)}\right| \leqslant\left(C L^{-1 / 2} \varepsilon+C(L) \varepsilon_{1} \varepsilon\right)$, and hence

$$
\begin{aligned}
& \left|\frac{d}{d s}\left(\int_{\Sigma_{s}}\left\langle Z^{(a)}, Z^{(b)}\right\rangle_{g(t)} d \mu_{g(t)}\right)-\frac{2 H}{\operatorname{area}_{g(t)}\left(\Sigma_{s}\right)} \int_{\Sigma_{s}}\left\langle Z^{(a)}, Z^{(b)}\right\rangle_{g(t)} d \mu_{g(t)}\right| \\
& \quad \leqslant\left(C L^{-1 / 2} \varepsilon+C(L) \varepsilon_{1} \varepsilon\right) .
\end{aligned}
$$

Thus, we conclude that

$$
\left|\frac{d}{d s}\left(\operatorname{area}_{g(t)}\left(\Sigma_{s}\right)^{-2} \int_{\Sigma_{s}}\left\langle Z^{(a)}, Z^{(b)}\right\rangle_{g(t)} d \mu_{g(t)}\right)\right| \leqslant C L^{-1 / 2} \varepsilon+C(L) \varepsilon_{1} \varepsilon .
$$

Step 14. The estimate in Step 13 implies that there exists a symmetric $3 \times 3$ matrix $Q_{a b}$ (independent of $\Sigma$ ) such that

$$
\left|Q_{a b}-\operatorname{area}_{g(-1)}(\Sigma)^{-2} \int_{\Sigma}\left\langle Z^{(a)}, Z^{(b)}\right\rangle_{g(-1)} d \mu_{g(-1)}\right| \leqslant C L^{-1 / 2} \varepsilon+C(L) \varepsilon_{1} \varepsilon
$$

whenever $\Sigma \subset\{|z| \leqslant 300\}$ is a leaf of the CMC foliation of $(M, g(-1))$. Moreover, since the vector fields $Z^{(1)}, Z^{(2)}$, and $Z^{(3)}$ are close to the standard rotation vector fields on the cylinder, up to some constant factor, the eigenvalues of the matrix $Q_{a b}$ lie in the interval $[1 / C, C]$ for some fixed constant $C$. The estimate for the Ricci tensor in Step 11 gives

$$
\left|\frac{d}{d t}\left(\operatorname{area}_{g(t)}(\Sigma)^{-2} \int_{\Sigma}\left\langle Z^{(a)}, Z^{(b)}\right\rangle_{g(t)} d \mu_{g(t)}\right)\right| \leqslant C L^{-1 / 2} \varepsilon+C(L) \varepsilon_{1} \varepsilon
$$

if $t \in[-200,-1]$ and $\Sigma \subset\{|z| \leqslant 300\}$ is a fixed leaf of the CMC foliation of $(M, g(-1))$. Consequently,

$$
\left|Q_{a b}-\operatorname{area}_{g(t)}(\Sigma)^{-2} \int_{\Sigma}\left\langle Z^{(a)}, Z^{(b)}\right\rangle_{g(t)} d \mu_{g(t)}\right| \leqslant C L^{-1 / 2} \varepsilon+C(L) \varepsilon_{1} \varepsilon
$$

whenever $t \in[-200,-1]$ and $\Sigma \subset\{|z| \leqslant 300\}$ is a leaf of the CMC foliation of $(M, g(-1))$. 
By Step 12, every leaf of the CMC foliation of $(M, g(t))$ which is contained in the region $\{|z| \leqslant 200\}$ is $\left(C L^{-1 / 2}+C(L) \varepsilon_{1} \varepsilon\right)$-close in the $C^{1}$-norm to a leaf of the CMC foliation of $(M, g(-1))$. This finally implies that

$$
\left|Q_{a b}-\operatorname{area}_{g(t)}(\Sigma)^{-2} \int_{\Sigma}\left\langle Z^{(a)}, Z^{(b)}\right\rangle_{g(t)} d \mu_{g(t)}\right| \leqslant C L^{-1 / 2} \varepsilon+C(L) \varepsilon_{1} \varepsilon
$$

whenever $t \in[-200,-1]$ and $\Sigma \subset\{|z| \leqslant 200\}$ is a leaf of the CMC foliation of $(M, g(t))$. Note that the matrix $Q_{a b}$ is independent of $t$ and independent of $\Sigma$.

By considering the vector fields $\sum_{b=1}^{3}\left(Q^{-1 / 2}\right)_{a b} Z^{(b)}$, we see that the point $\left(x_{0},-1\right)$ is $\left(C L^{-1 / 2} \varepsilon+C(L) \varepsilon_{1} \varepsilon\right)$-symmetric. Hence, if we choose $L$ sufficiently large and $\varepsilon_{1}$ sufficiently small (depending on $L$ ), then $\left(x_{0},-1\right)$ is $\frac{1}{2} \varepsilon$-symmetric.

\section{Rotational symmetry of ancient $\kappa$-solutions in dimension 3}

In this section, we give the proof of Theorem 1.2. Throughout this section, we assume that $(M, g(t)), t \in(-\infty, 0]$, is a 3 -dimensional ancient $\kappa$-solution which is non-compact and has positive sectional curvature. Our goal is to show that $(M, g(t))$ is rotationally symmetric. For each $t$, we denote by $R_{\max }(t)$ the supremum of the scalar curvature of $(M, g(t))$. By Perelman's pointwise derivative estimate [21], the function $t \mapsto R_{\max }(t)^{-1}$ is uniformly Lipschitz continuous.

Let us fix a large constant $L$ and a small constant $\varepsilon_{1}$ such that the conclusion of the neck improvement theorem holds. We assume that $\varepsilon_{1}$ is chosen small enough so that the results in $\S 7$ can be applied on every $\varepsilon_{1}$-neck. For each point $(x, t)$ in space-time, we denote by $\lambda_{1}(x, t)$ the smallest eigenvalue of the Ricci tensor at $(x, t)$. The following is a direct consequence of Perelman's work.

Proposition 9.1. Given $\varepsilon_{1}$, we can find a small positive constant $\theta$ (depending on $\left.\varepsilon_{1}\right)$ with the following property. Suppose that $(\bar{x}, \bar{t})$ is a point in space-time satisfying $\lambda_{1}(\bar{x}, \bar{t}) \leqslant \theta R(\bar{x}, \bar{t})$. Then, $(\bar{x}, \bar{t})$ lies at the center of an evolving $\varepsilon_{1}$-neck. Moreover, if $x$ lies outside the compact domain bounded by the leaf of the CMC foliation passing through $(\bar{x}, \bar{t})$, then $(x, \bar{t})$ lies at the center of an evolving $\varepsilon_{1}$-neck.

Proof. By Theorem A.2 and Corollary A.3, we can find a domain $\Omega_{\bar{t}}$ with the following properties:

- if $x \in M \backslash \Omega_{\bar{t}}$, then $(x, \bar{t})$ lies at the center of an evolving $\varepsilon_{1}$-neck;

- $\operatorname{diam}_{g(\bar{t})}\left(\Omega_{\bar{t}}\right) \leqslant C_{0} R_{\max }(\bar{t})^{-1 / 2}$, where $C_{0}$ is a large constant that depends on $\varepsilon_{1}$.

Now, if we choose $\theta$ sufficiently small, then every point $(\bar{x}, \bar{t})$ satisfying

$$
\lambda_{1}(\bar{x}, \bar{t}) \leqslant \theta R(\bar{x}, \bar{t})
$$


lies at the center of a neck $N$ of length $10 C_{0} R(\bar{x}, \bar{t})^{-1 / 2}$, and furthermore every point on $N$ lies at the center of an evolving $\varepsilon_{1}$-neck. The complement $M \backslash N$ has two connected components, one of which is bounded and one of which is unbounded. The bounded connected component of $M \backslash N$ must contain a point which does not lie at the center of an evolving $\varepsilon_{1}$-neck. If the unbounded connected component of $M \backslash N$ also contains a point which does not lie at the center of an evolving $\varepsilon_{1}$-neck, then

$$
\operatorname{diam}_{g(\bar{t})}\left(\Omega_{\bar{t}}\right) \geqslant 4 C_{0} R(\bar{x}, \bar{t})^{-1 / 2} \geqslant 4 C_{0} R_{\max }(\bar{t})^{-1 / 2},
$$

which is a contradiction. Consequently, every point in the unbounded connected component of $M \backslash N$ must lie at the center of an evolving $\varepsilon_{1}$-neck. From this, the assertion follows easily.

In the following, we fix $\theta$ so that the conclusion of Proposition 9.1 holds.

Definition 9.2. We say that the flow is $\varepsilon$-symmetric at time $\bar{t}$ if there exist a compact domain $D \subset M$ and time-independent vector fields $U^{(1)}, U^{(2)}$, and $U^{(3)}$ which are defined on an open set containing $D$ such that the following statements hold:

- there exists a point $x \in \partial D$ such that $\lambda_{1}(x, \bar{t})<\theta R(x, \bar{t})$;

- for each $x \in D$, we have $\lambda_{1}(x, \bar{t})>\frac{1}{2} \theta R(x, \bar{t})$;

- the boundary $\partial D$ is a leaf of the CMC foliation of $(M, g(\bar{t}))$;

- for each $x \in M \backslash D$, the point $(x, \bar{t})$ is $\varepsilon$-symmetric in the sense of Definition 8.2;

$$
\sup _{D \times\left[\bar{t}-R_{\max }(\bar{t})^{-1}, \bar{t}\right]} \sum_{l=0}^{2} \sum_{a=1}^{3} R_{\max }(\bar{t})^{-l}\left|D^{l}\left(\mathscr{L}_{U^{(a)}}(g(t))\right)\right|^{2} \leqslant \varepsilon^{2} ;
$$

- if $\Sigma \subset D$ is a leaf of the CMC foliation of $(M, g(\bar{t}))$ satisfying

$$
\sup _{x \in \Sigma} d_{g(\bar{t})}(x, \partial D) \leqslant 10 \operatorname{area}_{g(\bar{t})}(\partial D)^{1 / 2},
$$

then

$$
\sup _{\Sigma} \sum_{a=1}^{3} R_{\max }(\bar{t})\left|\left\langle U^{(a)}, \nu\right\rangle\right|^{2} \leqslant \varepsilon^{2}
$$

where $\nu$ denotes the unit normal vector to $\Sigma$ in $(M, g(\bar{t}))$;

- if $\Sigma \subset D$ is a leaf of the CMC foliation of $(M, g(\bar{t}))$ satisfying

$$
\sup _{x \in \Sigma} d_{g(\bar{t})}(x, \partial D) \leqslant 10 \operatorname{area}_{g(\bar{t})}(\partial D)^{1 / 2},
$$

then

$$
\sum_{a, b=1}^{3}\left|\delta_{a b}-\operatorname{area}_{g(\bar{t})}(\Sigma)^{-2} \int_{\Sigma}\left\langle U^{(a)}, U^{(b)}\right\rangle_{g(\bar{t})} d \mu_{g(\bar{t})}\right|^{2} \leqslant \varepsilon^{2} .
$$


Remark 9.3. For each $x \in M \backslash D$, the point $(x, \bar{t})$ lies at the center of an evolving $\varepsilon_{1}$-neck by Proposition 9.1.

Remark 9.4. Since $D \subset\left\{x \in M: \lambda_{1}(x, \bar{t})>\frac{1}{2} \theta R(x, \bar{t})\right\}$, Corollary A.3 implies that

$$
\operatorname{diam}_{g(\bar{t})}(D) \leqslant C R_{\max }(\bar{t})^{-1 / 2} \text { and } \quad \frac{1}{C} R_{\max }(\bar{t}) \leqslant R(x, \bar{t}) \leqslant R_{\max }(\bar{t}) \text { for all } x \in D .
$$

Here, $C$ is a large constant that depends on $\theta$. By Lemma 7.1, the vector fields $U^{(1)}$, $U^{(2)}$, and $U^{(3)}$ satisfy

$$
\sup _{D} \sum_{a=1}^{3}\left|U^{(a)}\right|^{2} \leqslant C R_{\max }(\bar{t})^{-1}
$$

where the norm is computed with respect to $g(\bar{t})$.

Lemma 9.5. Suppose that the flow is $\varepsilon$-symmetric at time $\bar{t}$. If $\tilde{t}$ is sufficiently close to $\bar{t}$, then the flow is $2 \varepsilon$-symmetric at time $\bar{t}$.

Proof. As the flow is $\varepsilon$-symmetric at time $\bar{t}$, we can find a compact domain $D \subset M$ and time-independent vector fields $U^{(1)}, U^{(2)}$, and $U^{(3)}$ which satisfy the conditions in Definition 9.2. In particular, every point in $(M \backslash D) \times\{\bar{t}\}$ is $\varepsilon$-symmetric.

By continuity, we can find a slightly larger domain $D_{1}$ with the following properties:

- there exists a point $x \in \partial D_{1}$ such that $\lambda_{1}(x, \bar{t})<\theta R(x, \bar{t})$;

- for each $x \in D_{1}$, we have $\lambda_{1}(x, \bar{t})>\frac{1}{2} \theta R(x, \bar{t})$;

- the boundary $\partial D_{1}$ is a leaf of the CMC foliation of $(M, g(\bar{t}))$;

$$
\sup _{D_{1} \times\left[\bar{t}-R_{\max }(\bar{t})^{-1}, \bar{t}\right]} \sum_{l=0}^{2} \sum_{a=1}^{3} R_{\max }(\bar{t})^{-l}\left|D^{l}\left(\mathscr{L}_{U^{(a)}}(g(t))\right)\right|^{2} \leqslant 2 \varepsilon^{2} ;
$$

- if $\Sigma \subset D_{1}$ is a leaf of the CMC foliation of $(M, g(\bar{t}))$ satisfying

$$
\sup _{x \in \Sigma} d_{g(\bar{t})}\left(x, \partial D_{1}\right) \leqslant 10 \operatorname{area}_{g(\bar{t})}\left(\partial D_{1}\right)^{1 / 2},
$$

then

$$
\sup _{\Sigma} \sum_{a=1}^{3} R_{\max }(\bar{t})\left|\left\langle U^{(a)}, \nu\right\rangle\right|^{2} \leqslant 2 \varepsilon^{2},
$$

where $\nu$ denotes the unit normal vector to $\Sigma$ in $(M, g(\bar{t}))$;

- if $\Sigma \subset D_{1}$ is a leaf of the CMC foliation of $(M, g(\bar{t}))$ satisfying

$$
\sup _{x \in \Sigma} d_{g(\bar{t})}\left(x, \partial D_{1}\right) \leqslant 10 \operatorname{area}_{g(\bar{t})}\left(\partial D_{1}\right)^{1 / 2},
$$

then

$$
\sum_{a, b=1}^{3}\left|\delta_{a b}-\operatorname{area}_{g(\bar{t})}(\Sigma)^{-2} \int_{\Sigma}\left\langle U^{(a)}, U^{(b)}\right\rangle_{g(\bar{t})} d \mu_{g(\bar{t})}\right|^{2} \leqslant 2 \varepsilon^{2} .
$$


Let $D_{0}$ be a compact domain with the property that $\partial D_{0}$ is a leaf of the CMC foliation and $\partial D_{0}$ lies in between $\partial D$ and $\partial D_{1}$. Using Lemma 8.3, we can find an open interval $I$ containing $\bar{t}$ such that every point on $\left(M \backslash D_{0}\right) \times I$ is $2 \varepsilon$-symmetric. Moreover, if $\tilde{t}$ is sufficiently close to $\bar{t}$, then we can find a domain $\widetilde{D}$ close to $D_{1}$ with the following properties:

- $D_{0} \subset \widetilde{D}$

- there exists a point $x \in \partial \widetilde{D}$ such that $\lambda_{1}(x, \tilde{t})<\theta R(x, \tilde{t})$;

- for each $x \in \widetilde{D}$, we have $\lambda_{1}(x, \tilde{t})>\frac{1}{2} \theta R(x, \tilde{t})$;

- the boundary $\partial \widetilde{D}$ is a leaf of the CMC foliation of $(M, g(\tilde{t}))$;

$$
\sup _{\widetilde{D} \times\left[\tilde{t}-R_{\max }(\tilde{t})^{-1}, \tilde{t}\right]} \sum_{l=0}^{2} \sum_{a=1}^{3} R_{\max }(\tilde{t})^{-l}\left|D^{l}\left(\mathscr{L}_{U^{(a)}}(g(t))\right)\right|^{2} \leqslant 4 \varepsilon^{2} ;
$$

- if $\Sigma \subset \widetilde{D}$ is a leaf of the CMC foliation of $(M, g(\tilde{t}))$ satisfying

$$
\sup _{x \in \Sigma} d_{g(\tilde{t})}(x, \partial \widetilde{D}) \leqslant 10 \operatorname{area}_{g(\tilde{t})}(\partial \widetilde{D})^{1 / 2},
$$

then

$$
\sup _{\Sigma} \sum_{a=1}^{3} R_{\max }(\tilde{t})\left|\left\langle U^{(a)}, \nu\right\rangle\right|^{2} \leqslant 4 \varepsilon^{2}
$$

where $\nu$ denotes the unit normal vector to $\Sigma$ in $(M, g(\tilde{t}))$;

- if $\Sigma \subset \widetilde{D}$ is a leaf of the CMC foliation of $(M, g(\tilde{t}))$ satisfying

$$
\sup _{x \in \Sigma} d_{g(\tilde{t})}(x, \partial \widetilde{D}) \leqslant 10 \operatorname{area}_{g(\tilde{t})}(\partial \widetilde{D})^{1 / 2},
$$

then

$$
\sum_{a, b=1}^{3}\left|\delta_{a b}-\operatorname{area}_{g(\tilde{t})}(\Sigma)^{-2} \int_{\Sigma}\left\langle U^{(a)}, U^{(b)}\right\rangle_{g(\tilde{t})} d \mu_{g(\tilde{t})}\right|^{2} \leqslant 4 \varepsilon^{2} .
$$

Therefore, if $\tilde{t}$ is sufficiently close to $\bar{t}$, then the flow is $2 \varepsilon$-symmetric at time $\tilde{t}$.

LEMma 9.6. Let us fix a time $\bar{t}$. Suppose that, for each $\varepsilon>0$, the flow is $\varepsilon$-symmetric at time $\bar{t}$. Then the manifold $(M, g(\bar{t}))$ is rotationally symmetric.

Proof. We consider the vector fields in Definition 9.2, and pass to the limit as $\varepsilon \rightarrow 0$. Hence, we can find a compact domain $D \subset M$ and vector fields $U^{(1)}, U^{(2)}$, and $U^{(3)}$ on $D$ with the following properties:

- the boundary $\partial D$ is a leaf of the CMC foliation of $(M, g(\bar{t}))$;

- the metric $g(\bar{t})$ is rotationally symmetric on $M \backslash D$;

- the vector fields $U^{(a)}$ satisfy $\mathscr{L}_{U^{(a)}}(g(\bar{t}))=0$ in $D$; 
- the vector fields $U^{(a)}$ are tangential along $\partial D$;

$$
\operatorname{area}_{g(\bar{t})}(\partial D)^{-2} \int_{\partial D}\left\langle U^{(a)}, U^{(b)}\right\rangle_{g(\bar{t})} d \mu_{g(\bar{t})}=\delta_{a b} .
$$

In particular, we have that $(\partial D, g(\bar{t}))$ is a round sphere, and every Killing vector field on $(\partial D, g(\bar{t}))$ can be extended to a Killing vector field on $(D, g(\bar{t}))$. This implies that the metric $g(\bar{t})$ is rotationally symmetric in $D$.

We now proceed with the proof of Theorem 1.2. We first show that we can find a sequence of times where the solution is arbitrarily close to the Bryant soliton. This argument relies on the Harnack inequality together with the classification of steady gradient Ricci solitons in [8].

Proposition 9.7. We can find a sequence of times $\hat{t}_{k} \rightarrow-\infty$ and a sequence of points $\hat{p}_{k} \in M$ with the following property. If we perform a parabolic rescaling around the point $\left(\hat{p}_{k}, \hat{t}_{k}\right)$ by the factor $R_{\max }\left(\hat{t}_{k}\right)^{1 / 2}$, then the rescaled flows converge to the Bryant soliton in the Cheeger-Gromov sense. Moreover, the points $\hat{p}_{k}$ converge to the tip of the Bryant soliton, and we have

$$
\frac{R\left(\hat{p}_{k}, \hat{t}_{k}\right)}{R_{\max }\left(\hat{t}_{k}\right)} \rightarrow 1 \quad \text { as } k \rightarrow \infty
$$

Proof. By [25], $(M, g(t))$ is a type-II ancient solution, i.e.

$$
\sup _{(x, t) \in M \times(-\infty, 0]}(-t) R(x, t)=\infty .
$$

We now argue as in $[17, \S 16]$ to extract a type-II blow-up limit. For $k$ large, we choose a point $\left(\hat{p}_{k}, \hat{t}_{k}\right) \in M \times(-k, 0)$ with the property that

$$
\sup _{(x, t) \in M \times(-k, 0)}\left(1+\frac{t}{k}\right)(-t) R(x, t) \leqslant\left(1+\frac{1}{k}\right)\left(1+\frac{\hat{t}_{k}}{k}\right)\left(-\hat{t}_{k}\right) R\left(\hat{p}_{k}, \hat{t}_{k}\right) .
$$

In particular,

$$
R_{\max }\left(\hat{t}_{k}\right) \leqslant\left(1+\frac{1}{k}\right) R\left(\hat{p}_{k}, \hat{t}_{k}\right)
$$

Since $(M, g(t))$ is a type-II ancient solution, we know that

$$
\sup _{(x, t) \in M \times(-k, 0)}\left(1+\frac{t}{k}\right)(-t) R(x, t) \rightarrow \infty,
$$

and hence

$$
\left(1+\frac{\hat{t}_{k}}{k}\right)\left(-\hat{t}_{k}\right) R\left(\hat{p}_{k}, \hat{t}_{k}\right) \rightarrow \infty
$$


This implies that $\left(-\hat{t}_{k}\right) R\left(\hat{p}_{k}, \hat{t}_{k}\right) \rightarrow \infty,\left(k+\hat{t}_{k}\right) R\left(\hat{p}_{k}, \hat{t}_{k}\right) \rightarrow \infty$, and

$$
\limsup _{k \rightarrow \infty} \sup _{(x, t) \in M \times\left[\hat{t}_{k}-A R\left(\hat{p}_{k}, \hat{t}_{k}\right)^{-1}, \hat{t}_{k}+A R\left(\hat{p}_{k}, \hat{t}_{k}\right)^{-1}\right]} \frac{R(x, t)}{R\left(\hat{p}_{k}, \hat{t}_{k}\right)} \leqslant 1
$$

for every fixed $A$.

We now rescale around the point $\left(\hat{p}_{k}, \hat{t}_{k}\right)$ by the factor $R\left(\hat{p}_{k}, \hat{t}_{k}\right)^{1 / 2}$. Passing to the limit as $k \rightarrow \infty$, we obtain an eternal solution to the Ricci flow which is complete; $\kappa$-noncollapsed; has non-negative sectional curvature; and has scalar curvature at most 1 at each point in space-time. Moreover, there exists a point on the limiting solution where the scalar curvature is equal to 1 . Therefore, the limiting solution attains equality in Hamilton's Harnack inequality [16], and consequently must be a steady gradient Ricci soliton [15]. By [8], the limit flow must be the Bryant soliton.

CoRollary 9.8. There exists a sequence $\hat{\varepsilon}_{k} \rightarrow 0$ with the following properties. For each $t \in\left[\hat{t}_{k}-\hat{\varepsilon}_{k}^{-2} R_{\max }\left(\hat{t}_{k}\right)^{-1}, \hat{t}_{k}\right]$, we have

$$
\left(1-\hat{\varepsilon}_{k}\right) R_{\max }\left(\hat{t}_{k}\right) \leqslant R\left(\hat{p}_{k}, t\right) \leqslant R_{\max }(t) \leqslant R_{\max }\left(\hat{t}_{k}\right) .
$$

Moreover, for each $t \in\left[\hat{t}_{k}-\hat{\varepsilon}_{k}^{-2} R_{\max }\left(\hat{t}_{k}\right)^{-1}, \hat{t}_{k}\right]$, the flow is $\hat{\varepsilon}_{k}$-symmetric at time $t$.

Proof. The Harnack inequality (cf. [16]) implies $R_{\max }(t) \leqslant R_{\max }\left(\hat{t}_{k}\right)$ for each $t \leqslant \hat{t}_{k}$. The remaining statements follow by combining Proposition 9.7 and Theorem A.2.

From now on, we assume that the ancient solution $(M, g(t))$ is not rotationally symmetric. In view of Corollary A.3, we can find a sequence of positive real numbers $\varepsilon_{k}$ with the following properties:

- $\varepsilon_{k} \rightarrow 0$;

- $\varepsilon_{k} \geqslant 2 \hat{\varepsilon}_{k}$

- if a point $(x, t)$ in space-time satisfies $R(x, t) \leqslant \hat{\varepsilon}_{k} R_{\max }(t)$, then $(x, t)$ lies at the center of an evolving $\varepsilon_{k}^{2}$-neck.

For each $k$, we define

$$
t_{k}=\inf \left\{t \in\left[\hat{t}_{k}, 0\right]: \text { the flow is not } \varepsilon_{k} \text {-symmetric at time } t\right\} .
$$

For abbreviation, let $R_{\max }\left(t_{k}\right)=r_{k}^{-2}$. The Harnack inequality [16] implies $R_{\max }(t) \leqslant r_{k}^{-2}$ for all $t \leqslant t_{k}$.

Lemma 9.9. If $t \in\left[\hat{t}_{k}-\hat{\varepsilon}_{k}^{-2} R_{\max }\left(\hat{t}_{k}\right)^{-1}, t_{k}\right)$, then the flow is $\varepsilon_{k}$-symmetric at time $t$. In particular, if $(x, t) \in M \times\left[\hat{t}_{k}-\hat{\varepsilon}_{k}^{-2} R_{\max }\left(\hat{t}_{k}\right)^{-1}, t_{k}\right)$ is a point in space-time satisfying $\lambda_{1}(x, t)<\frac{1}{2} \theta R(x, t)$, then the point $(x, t)$ is $\varepsilon_{k}$-symmetric. 
Proof. The first statement follows immediately from the definition of $t_{k}$. The second statement follows from the first statement, keeping in mind Definition 9.2.

LEMma 9.10. The sequence $t_{k}$ satisfies $\lim _{k \rightarrow \infty} t_{k}=-\infty$.

Proof. Suppose that $\limsup _{k \rightarrow \infty} t_{k}>-\infty$. Let us now consider an arbitrary time $\bar{t}<\lim \sup _{k \rightarrow \infty} t_{k}$. Then, there exist arbitrarily large integers $k$ with the property that $\bar{t} \in\left[\hat{t}_{k}, t_{k}\right)$. By Lemma 9.9, there exist arbitrarily large integers $k$ with the property that the flow is $\varepsilon_{k}$-symmetric at time $\bar{t}$. Since $\varepsilon_{k} \rightarrow 0$, Lemma 9.6 implies that $(M, g(\bar{t}))$ is rotationally symmetric.

To summarize, we have shown that the solution $(M, g(t))$ is rotationally symmetric for all $t<\lim \sup _{k \rightarrow \infty} t_{k}$. By the uniqueness result in [12], the solution is rotationally symmetric for all $t$, contrary to our assumption.

In the next step, we show that, at time $t_{k}$, the solution is close to the Bryant soliton. This argument relies in a crucial way on Theorem 1.1.

Proposition 9.11. There exists a sequence of points $p_{k} \in M$ with the following properties. If we perform a parabolic rescaling around the point $\left(p_{k}, t_{k}\right)$ by the factor $R_{\max }\left(t_{k}\right)^{1 / 2}=r_{k}^{-1}$, then the rescaled flows converge to the Bryant soliton in the CheegerGromov sense. Moreover, the points $p_{k}$ converge to the tip of the Bryant soliton, and we have $r_{k}^{2} R\left(p_{k}, t_{k}\right) \rightarrow 1$ as $k \rightarrow \infty$.

Proof. For each $k$, the manifold $\left(M, g\left(t_{k}\right)\right)$ contains a point which does not lie on a neck. Hence, we can find a sequence of points $q_{k} \in M$ such that

$$
\liminf _{k \rightarrow \infty} \frac{\lambda_{1}\left(q_{k}, t_{k}\right)}{R\left(q_{k}, t_{k}\right)}>0 .
$$

By Corollary A.3, $\liminf _{k \rightarrow \infty} r_{k}^{2} R\left(q_{k}, t_{k}\right)>0$. This implies that

$$
\liminf _{k \rightarrow \infty} r_{k}^{2} \lambda_{1}\left(q_{k}, t_{k}\right)>0
$$

We now rescale the flow $(M, g(t))$ around the point $\left(q_{k}, t_{k}\right)$ by the factor $r_{k}^{-1}$. Passing to the limit as $k \rightarrow \infty$, we obtain a non-compact ancient $\kappa$-solution $\left(M^{\infty}, g^{\infty}(s)\right)$. Since

$$
\liminf _{k \rightarrow \infty} r_{k}^{2} \lambda_{1}\left(q_{k}, t_{k}\right)>0
$$

the limit manifold $\left(M^{\infty}, g^{\infty}(0)\right)$ does not split off a line. By the uniqueness result in [12], the manifold $\left(M^{\infty}, g^{\infty}(s)\right)$ does not split off a line for any $s \leqslant 0$. By the strict maximum principle, the limit flow $\left(M^{\infty}, g^{\infty}(s)\right)$ has positive sectional curvature for each $s \leqslant 0$. 
We claim that the limiting flow $\left(M^{\infty}, g^{\infty}(s)\right)$ is rotationally symmetric. To prove this, we fix an arbitrary time $\bar{s}<0$. Since $R_{\max }\left(\hat{t}_{k}\right) \leqslant r_{k}^{-2}$, it follows that

$$
t_{k}+r_{k}^{2} \bar{s} \in\left[\hat{t}_{k}-\hat{\varepsilon}_{k}^{-2} R_{\max }\left(\hat{t}_{k}\right)^{-1}, t_{k}\right)
$$

if $k$ is sufficiently large. By Lemma 9.9, the original flow is $\varepsilon_{k}$-symmetric at time $t_{k}+r_{k}^{2} \bar{s}$, provided that $k$ is sufficiently large. By the Harnack inequality,

$$
R_{\max }\left(t_{k}+r_{k}^{2} \bar{s}\right) \leqslant r_{k}^{-2} .
$$

On the other hand, since $\left(M^{\infty}, g^{\infty}(\bar{s})\right)$ has positive sectional curvature, we obtain

$$
\liminf _{k \rightarrow \infty} r_{k}^{2} \lambda_{1}\left(q_{k}, t_{k}+r_{k}^{2} \bar{s}\right)>0 .
$$

Therefore, the cap in $\left(M, g\left(t_{k}+r_{k}^{2} \bar{s}\right)\right)$ has diameter $\lesssim r_{k}$, the scalar curvature on the cap is $\sim r_{k}^{-2}$, and the cap has distance $\lesssim r_{k}$ from the point $q_{k}$. We now pass to the limit as $k \rightarrow \infty$. In the limit, we obtain a domain $D^{\infty} \subset M^{\infty}$ and vector fields $U^{(\infty, 1)}, U^{(\infty, 2)}$, and $U^{(\infty, 3)}$ on $D^{\infty}$ with the following properties:

- the boundary $\partial D^{\infty}$ is a leaf of the CMC foliation of $\left(M^{\infty}, g^{\infty}(\bar{s})\right)$;

- the metric $g(\bar{s})$ is rotationally symmetric on $M^{\infty} \backslash D^{\infty}$;

- the vector fields $U^{(\infty, a)}$ satisfy $\mathscr{L}_{U^{(\infty, a)}}\left(g^{\infty}(\bar{s})\right)=0$ in $D^{\infty}$;

- the vector fields $U^{(\infty, a)}$ are tangential along $\partial D^{\infty}$;

$$
\operatorname{area}_{g^{\infty}(\bar{s})}\left(\partial D^{\infty}\right)^{-2} \int_{\partial D^{\infty}}\left\langle U^{(\infty, a)}, U^{(\infty, b)}\right\rangle_{g^{\infty}(\bar{s})} d \mu_{g^{\infty}(\bar{s})}=\delta_{a b} .
$$

Thus, we conclude that the limiting manifold $\left(M^{\infty}, g^{\infty}(\bar{s})\right)$ is rotationally symmetric.

To summarize, we have shown that $\left(M^{\infty}, g^{\infty}(s)\right)$ is a non-compact ancient $\kappa$-solution which is rotationally symmetric and has positive sectional curvature. By Theorem 1.1, the limiting flow $\left(M^{\infty}, g^{\infty}(s)\right)$ must be isometric to the Bryant soliton, up to scaling.

Finally, we claim that $R_{g^{\infty}(0)}\left(p_{\infty}\right)=1$, where $p_{\infty} \in M^{\infty}$ denotes the tip of the limiting soliton $\left(M^{\infty}, g^{\infty}(s)\right)$. To see this, consider a sequence of points $p_{k} \in M$ converging to $p_{\infty}$. Clearly,

$$
R_{g^{\infty}(0)}\left(p_{\infty}\right)=\lim _{k \rightarrow \infty} r_{k}^{2} R\left(p_{k}, t_{k}\right) \in(0,1] .
$$

Using Proposition A.1, we can find a large constant $A$ such that

$$
\sup _{x \in M \backslash B_{g\left(t_{k}\right)}\left(p_{k}, A R\left(p_{k}, t_{k}\right)^{-1 / 2}\right)} \frac{R\left(x, t_{k}\right)}{R\left(p_{k}, t_{k}\right)} \leqslant \frac{1}{2}
$$

if $k$ is sufficiently large. Moreover, since the scalar curvature of $\left(M^{\infty}, g^{\infty}(0)\right)$ attains its maximum at the point $p_{\infty}$, we obtain

$$
\limsup _{k \rightarrow \infty} \sup _{x \in B_{g\left(t_{k}\right)}\left(p_{k}, A R\left(p_{k}, t_{k}\right)^{-1 / 2}\right)} \frac{R\left(x, t_{k}\right)}{R\left(p_{k}, t_{k}\right)} \leqslant 1
$$


for every fixed $A$. Putting these facts together, we conclude that

$$
\limsup _{k \rightarrow \infty} \sup _{x \in M} \frac{R\left(x, t_{k}\right)}{R\left(p_{k}, t_{k}\right)} \leqslant 1 \text {. }
$$

Thus,

$$
R_{g^{\infty}(0)}\left(p_{\infty}\right)=\lim _{k \rightarrow \infty} r_{k}^{2} R\left(p_{k}, t_{k}\right) \geqslant 1 .
$$

COROLlaRY 9.12. There exists a sequence of positive real numbers $\delta_{k} \rightarrow 0$ such that $\delta_{k} \geqslant 2 \varepsilon_{k}$ for each $k$ and the following statements hold when $k$ is sufficiently large:

- for each $t \in\left[t_{k}-\delta_{k}^{-1} r_{k}^{2}, t_{k}\right]$, we have

$$
\frac{1}{3}\left(1-\delta_{k}\right) g \leqslant r_{k}^{2} \operatorname{Ric} \leqslant \frac{1}{3}\left(1+\delta_{k}\right) g
$$

at the point $\left(p_{k}, t\right)$;

- the scalar curvature satisfies

$$
\frac{1}{2 K}\left(r_{k}^{-1} d_{g(t)}\left(p_{k}, x\right)+1\right)^{-1} \leqslant r_{k}^{2} R(x, t) \leqslant 2 K\left(r_{k}^{-1} d_{g(t)}\left(p_{k}, x\right)+1\right)^{-1}
$$

for all points $(x, t) \in B_{g\left(t_{k}\right)}\left(p_{k}, \delta_{k}^{-1} r_{k}\right) \times\left[t_{k}-\delta_{k}^{-1} r_{k}^{2}, t_{k}\right]$;

- there exists a non-negative function $f: B_{g\left(t_{k}\right)}\left(p_{k}, \delta_{k}^{-1} r_{k}\right) \times\left[t_{k}-\delta_{k}^{-1} r_{k}^{2}, t_{k}\right] \rightarrow \mathbb{R}$ such that

$\left|\operatorname{Ric}-D^{2} f\right| \leqslant \delta_{k} r_{k}^{-2},\left.\quad|\Delta f+| \nabla f\right|^{2}-r_{k}^{-2} \mid \leqslant \delta_{k} r_{k}^{-2}, \quad$ and $\left.\quad\left|\frac{\partial}{\partial t} f+\right| \nabla f\right|^{2} \mid \leqslant \delta_{k} r_{k}^{-2}$

- the function $f$ satisfies

$$
\frac{1}{2 K}\left(r_{k}^{-1} d_{g(t)}\left(p_{k}, x\right)+1\right) \leqslant f(x, t)+1 \leqslant 2 K\left(r_{k}^{-1} d_{g(t)}\left(p_{k}, x\right)+1\right)
$$

for all points $(x, t) \in B_{g\left(t_{k}\right)}\left(p_{k}, \delta_{k}^{-1} r_{k}\right) \times\left[t_{k}-\delta_{k}^{-1} r_{k}^{2}, t_{k}\right]$.

Here, $K \geqslant 10$ is a universal constant.

Proof. On the Bryant soliton, the eigenvalues of the Ricci tensor at the tip are equal to $\frac{1}{3}$. Moreover, on the Bryant soliton, the scalar curvature satisfies

$$
\frac{1}{K}(d(p, x)+1)^{-1} \leqslant R \leqslant K(d(p, x)+1)^{-1},
$$

where $p$ denotes the tip of the Bryant soliton and $K$ is a universal constant. Furthermore, on the Bryant soliton, the potential function $f$ satisfies

$$
\frac{1}{K}(d(p, x)+1) \leqslant f+1 \leqslant K(d(p, x)+1),
$$

where again $p$ denotes the tip of the Bryant soliton and $K$ is a universal constant. Finally, the potential function $f$ satisfies

$$
\text { Ric }=D^{2} f, \quad \Delta f+|\nabla f|^{2}=1, \quad \text { and } \quad \frac{\partial}{\partial t} f+|\nabla f|^{2}=0 .
$$

The assertion now follows from Proposition 9.11. 
Corollary 9.13. For each $t \in\left[t_{k}-\delta_{k}^{-1} r_{k}^{2}, t_{k}\right]$, we have

$$
\left(1-\delta_{k}\right) r_{k}^{-2} \leqslant R\left(p_{k}, t\right) \leqslant R_{\max }(t) \leqslant r_{k}^{-2}
$$

Proof. The Harnack inequality (cf. [16]) implies that $R_{\max }(t) \leqslant r_{k}^{-2}$ for each $t \leqslant t_{k}$. Moreover, Corollary 9.12 implies $R\left(p_{k}, t\right) \geqslant\left(1-\delta_{k}\right) r_{k}^{-2}$ for each $t \in\left[t_{k}-\delta_{k}^{-1} r_{k}^{2}, t_{k}\right]$.

Lemma 9.14. The time derivative of the distance function satisfies

$$
0 \leqslant-\frac{d}{d t} d_{g(t)}\left(p_{k}, x\right) \leqslant 80 r_{k}^{-1}
$$

for all $(x, t) \in M \times\left[t_{k}-\delta_{k}^{-1} r_{k}^{2}, t_{k}\right]$.

Proof. Using Lemma 8.3(b) in [21], we obtain

$$
0 \leqslant-\frac{d}{d t} d_{g(t)}\left(p_{k}, x\right) \leqslant 80 R_{\max }(t)^{1 / 2} \leqslant 80 r_{k}^{-1}
$$

for all $(x, t) \in M \times\left[t_{k}-\delta_{k}^{-1} r_{k}^{2}, t_{k}\right]$.

In view of Theorem A.2 and Corollary 9.13, we can find a large constant $\Lambda$ with the following properties:

- $L \sqrt{4 K / \Lambda} \leqslant 10^{-6}$;

- if $(\bar{x}, \bar{t}) \in M \times\left[t_{k}-\delta_{k}^{-1} r_{k}^{2}, t_{k}\right]$ is a point in space-time satisfying $d_{g(\bar{t})}\left(p_{k}, \bar{x}\right) \geqslant \Lambda r_{k}$, then $\lambda_{1}(x, t)<\frac{1}{2} \theta R(x, t)$ for all points $(x, t) \in B_{g(\bar{t})}\left(\bar{x}, L R(\bar{x}, \bar{t})^{-1 / 2}\right) \times\left[\bar{t}-L R(\bar{x}, \bar{t})^{-1}, \bar{t}\right]$.

LEMMA 9.15. If $k$ is sufficiently large, then the following statement holds. If $(\bar{x}, \bar{t}) \in$ $M \times\left[t_{k}-\delta_{k}^{-1} r_{k}^{2}, t_{k}\right]$ satisfies $d_{g(\bar{t})}\left(p_{k}, \bar{x}\right) \geqslant \Lambda r_{k}$, then $(\bar{x}, \bar{t})$ is $\frac{1}{2} \varepsilon_{k}$-symmetric.

Proof. We distinguish two cases.

Case 1. Suppose first that $R(\bar{x}, \bar{t}) \leqslant \hat{\varepsilon}_{k} R_{\max }(\bar{t})$. By our choice of $\varepsilon_{k}$, the point $(\bar{x}, \bar{t})$ lies at the center of an evolving $\varepsilon_{k}^{2}$-neck, and this directly implies that $(\bar{x}, \bar{t})$ is $\frac{1}{2} \varepsilon_{k}$-symmetric.

Case 2. Suppose next that $R(\bar{x}, \bar{t}) \geqslant \hat{\varepsilon}_{k} R_{\max }(\bar{t})$. Note that Corollary 9.13 implies $R_{\max }(\bar{t}) \geqslant \frac{1}{2} r_{k}^{-2}$, and hence $R(\bar{x}, \bar{t}) \geqslant \frac{1}{2} \hat{\varepsilon}_{k} r_{k}^{-2}$. On the other hand, $R_{\max }\left(\hat{t}_{k}\right) \leqslant r_{k}^{-2}$. Hence, if $k$ is sufficiently large, then we obtain

$$
\begin{aligned}
\bar{t}-L R(\bar{x}, \bar{t})^{-1} & \geqslant t_{k}-\delta_{k}^{-1} r_{k}^{2}-2 L \hat{\varepsilon}_{k}^{-1} r_{k}^{2} \\
& \geqslant \hat{t}_{k}-\delta_{k}^{-1} R_{\max }\left(\hat{t}_{k}\right)^{-1}-2 L \hat{\varepsilon}_{k}^{-1} R_{\max }\left(\hat{t}_{k}\right)^{-1} \\
& \geqslant \hat{t}_{k}-\hat{\varepsilon}_{k}^{-2} R_{\max }\left(\hat{t}_{k}\right)^{-1}
\end{aligned}
$$


By definition of $\Lambda$, we have $\lambda_{1}(x, t)<\frac{1}{2} \theta R(x, t)$ for all points

$$
(x, t) \in B_{g(\bar{t})}\left(\bar{x}, L R(\bar{x}, \bar{t})^{-1 / 2}\right) \times\left[\bar{t}-L R(\bar{x}, \bar{t})^{-1}, \bar{t}\right] .
$$

Hence, by Proposition 9.1, every point in

$$
B_{g(\bar{t})}\left(\bar{x}, L R(\bar{x}, \bar{t})^{-1 / 2}\right) \times\left[\bar{t}-L R(\bar{x}, \bar{t})^{-1}, \bar{t}\right]
$$

lies at the center of an evolving $\varepsilon_{1}$-neck. Moreover, by Lemma 9.9, every point in

$$
B_{g(\bar{t})}\left(\bar{x}, L R(\bar{x}, \bar{t})^{-1 / 2}\right) \times\left[\bar{t}-L R(\bar{x}, \bar{t})^{-1}, \bar{t}\right)
$$

is $\varepsilon_{k}$-symmetric. Using the neck improvement theorem, we conclude that the point $(\bar{x}, \bar{t})$ is $\frac{1}{2} \varepsilon_{k}$-symmetric.

Proposition 9.16. If $k$ is sufficiently large, then the following statement holds. If $(\bar{x}, \bar{t}) \in M \times\left[t_{k}-2^{-j} \delta_{k}^{-1} r_{k}^{2}, t_{k}\right]$ satisfies $2^{j / 400} \Lambda r_{k} \leqslant d_{g(\bar{t})}\left(p_{k}, \bar{x}\right) \leqslant(400 K L)^{-j} \delta_{k}^{-1} r_{k}$, then $(\bar{x}, \bar{t})$ is $2^{-j-1} \varepsilon_{k}$-symmetric.

Proof. We argue by induction on $j$. For $j=0$, the assertion follows from Lemma 9.15.

We now assume that $j \geqslant 1$ and the assertion holds for $j-1$. We will show that the assertion holds for $j$. To that end, we consider a point $(\bar{x}, \bar{t}) \in M \times\left[t_{k}-2^{-j} \delta_{k}^{-1} r_{k}^{2}, t_{k}\right]$ such that $2^{j / 400} \Lambda r_{k} \leqslant d_{g(\bar{t})}\left(p_{k}, \bar{x}\right) \leqslant(400 K L)^{-j} \delta_{k}^{-1} r_{k}$. Clearly, $\lambda_{1}(\bar{x}, \bar{t})<\frac{1}{2} \theta R(\bar{x}, \bar{t})$ by definition of $\Lambda$. By Proposition 9.1, we have that $(\bar{x}, \bar{t})$ lies at the center of an evolving $\varepsilon_{1}$-neck. Let $R(\bar{x}, \bar{t})=r^{-2}$. We will show that every point in $B_{g(\bar{t})}(\bar{x}, L r) \times\left[\bar{t}-L r^{2}, \bar{t}\right]$ is $2^{-j} \varepsilon_{k}$-symmetric. By Corollary $9.12, r^{2} \leqslant 4 K r_{k} d_{g(\bar{t})}\left(p_{k}, \bar{x}\right)$. This implies that

$$
\begin{aligned}
\bar{t}-L r^{2} & \geqslant \bar{t}-4 K L r_{k} d_{g(\bar{t})}\left(p_{k}, \bar{x}\right) \\
& \geqslant \bar{t}-4 K L(400 K L)^{-j} \delta_{k}^{-1} r_{k}^{2} \\
& \geqslant \bar{t}-2^{-j} \delta_{k}^{-1} r_{k}^{2} \\
& \geqslant t_{k}-2^{-j+1} \delta_{k}^{-1} r_{k}^{2} .
\end{aligned}
$$

In the next step, we observe that

$$
r^{2} \leqslant 4 K r_{k} d_{g(\bar{t})}\left(p_{k}, \bar{x}\right) \leqslant \frac{4 K}{\Lambda} d_{g(\bar{t})}\left(p_{k}, \bar{x}\right)^{2} .
$$

Since $L \sqrt{4 K / \Lambda} \leqslant 10^{-6}$, we obtain

$$
r \leqslant \sqrt{\frac{4 K}{\Lambda}} d_{g(\bar{t})}\left(p_{k}, \bar{x}\right) \leqslant 10^{-6} L^{-1} d_{g(\bar{t})}\left(p_{k}, \bar{x}\right) .
$$


Consequently,

$$
\begin{aligned}
d_{g(\bar{t})}\left(p_{k}, x\right) & \geqslant d_{g(\bar{t})}\left(p_{k}, \bar{x}\right)-L r \\
& \geqslant\left(1-10^{-6}\right) d_{g(\bar{t})}\left(p_{k}, \bar{x}\right) \\
& \geqslant\left(1-10^{-6}\right) 2^{j / 400} \Lambda r_{k} \\
& \geqslant 2^{(j-1) / 400} \Lambda r_{k}
\end{aligned}
$$

for all $x \in B_{g(\bar{t})}(\bar{x}, L r)$. On the other hand, $r_{k}=R_{\max }\left(t_{k}\right)^{-1 / 2} \leqslant R_{\max }(\bar{t})^{-1 / 2} \leqslant r$ by the Harnack inequality. Using this, together with the inequality $r^{2} \leqslant 4 K r_{k} d_{g(\bar{t})}\left(p_{k}, \bar{x}\right)$, we obtain

$$
\begin{aligned}
d_{g(\bar{t})}\left(p_{k}, x\right)+80 L r^{2} r_{k}^{-1} & \leqslant d_{g(\bar{t})}\left(p_{k}, \bar{x}\right)+L r+80 L r^{2} r_{k}^{-1} \\
& \leqslant d_{g(\bar{t})}\left(p_{k}, \bar{x}\right)+81 L r^{2} r_{k}^{-1} \\
& \leqslant 400 K L d_{g(\bar{t})}\left(p_{k}, \bar{x}\right) \\
& \leqslant(400 K L)^{-j+1} \delta_{k}^{-1} r_{k}
\end{aligned}
$$

for all $x \in B_{g(\bar{t})}(\bar{x}, L r)$. Lemma 9.14 gives

$$
d_{g(\bar{t})}\left(p_{k}, x\right) \leqslant d_{g(t)}\left(p_{k}, x\right) \leqslant d_{g(\bar{t})}\left(p_{k}, x\right)+80 L r^{2} r_{k}^{-1}
$$

and hence

$$
2^{(j-1) / 400} \Lambda r_{k} \leqslant d_{g(t)}\left(p_{k}, x\right) \leqslant(400 K L)^{-j+1} \delta_{k}^{-1} r_{k}
$$

for all $(x, t) \in B_{g(\bar{t})}(\bar{x}, L r) \times\left[\bar{t}-L r^{2}, \bar{t}\right]$. Therefore, the induction hypothesis implies that every point in $B_{g(\bar{t})}(\bar{x}, L r) \times\left[\bar{t}-L r^{2}, \bar{t}\right]$ is $2^{-j} \varepsilon_{k}$-symmetric. Consequently, the point $(\bar{x}, \bar{t})$ is $2^{-j-1} \varepsilon_{k}$-symmetric by the neck improvement theorem.

Lemma 9.17. If $j$ is sufficiently large and $k$ is sufficiently large depending on $j$, then the following holds. Given any $\bar{t} \in\left[t_{k}-2^{j / 100} r_{k}^{2}, t_{k}\right]$, there exist time-independent vector fields $U^{(1)}, U^{(2)}$, and $U^{(3)}$ on $B_{g(\bar{t})}\left(p_{k}, 2^{j / 400} \Lambda r_{k}\right)$ with the following properties:

$$
\left|\mathscr{L}_{U^{(a)}}(g(t))\right|+r_{k}\left|D\left(\mathscr{L}_{U^{(a)}}(g(t))\right)\right| \leqslant C\left(r_{k}^{-1} d_{g(t)}\left(p_{k}, x\right)+1\right)^{-100} \varepsilon_{k}
$$

for all $(x, t) \in B_{g(\bar{t})}\left(p_{k}, 2^{j / 400} \Lambda r_{k}\right) \times\left[\bar{t}-r_{k}^{2}, \bar{t}\right]$;

- if $t \in\left[\bar{t}-r_{k}^{2}, \bar{t}\right]$ and $x \in B_{g(\bar{t})}\left(p_{k}, 2^{j / 400} \Lambda r_{k}\right) \backslash B_{g(\bar{t})}\left(p_{k}, 2 \Lambda r_{k}\right)$, then

$$
r_{k}^{-1}\left|\left\langle U^{(a)}, \nu\right\rangle\right| \leqslant C\left(r_{k}^{-1} d_{g(t)}\left(p_{k}, x\right)+1\right)^{-100} \varepsilon_{k},
$$

where $\nu$ denotes the unit normal to the CMC foliation of $(M, g(t))$; 
- if $t \in\left[\bar{t}-r_{k}^{2}, \bar{t}\right]$ and $x \in B_{g(\bar{t})}\left(p_{k}, 2^{j / 400} \Lambda r_{k}\right) \backslash B_{g(\bar{t})}\left(p_{k}, 2 \Lambda r_{k}\right)$, then

$$
\sum_{a, b=1}^{3}\left|\delta_{a b}-\operatorname{area}_{g(t)}(\Sigma)^{-2} \int_{\Sigma}\left\langle U^{(a)}, U^{(b)}\right\rangle_{g(t)} d \mu_{g(t)}\right| \leqslant C\left(r_{k}^{-1} d_{g(t)}\left(p_{k}, x\right)+1\right)^{-100} \varepsilon_{k},
$$

where $\Sigma$ denotes the leaf of the CMC foliation passing through $(x, t)$.

Moreover, on the ball $B_{g(\bar{t})}\left(p_{k}, 2^{j / 400} \Lambda r_{k}\right)$, the vector fields $U^{(1)}, U^{(2)}$, and $U^{(3)}$ are close to the standard rotation vector fields on the Bryant soliton in the $C^{2}$-norm.

Proof. We proceed in two steps.

Step 1. Suppose first that $\bar{t} \in\left[t_{k}-2^{j / 100} r_{k}^{2}, t_{k}\right)$. By Lemma 9.9, we have that the flow is $\varepsilon_{k}$-symmetric at time $\bar{t}$. Moreover, if $\bar{x} \in B_{g(\bar{t})}\left(p_{k}, 2^{j / 400} \Lambda r_{k}\right) \backslash B_{g(\bar{t})}\left(p_{k}, \Lambda r_{k}\right)$, then the point $(\bar{x}, \bar{t})$ is $C\left(r_{k}^{-1} d_{g(\bar{t})}\left(p_{k}, \bar{x}\right)\right)^{-400} \varepsilon_{k}$-symmetric by Proposition 9.16. By a repeated application of Corollary 7.5, we can construct vector fields $U^{(1)}, U^{(2)}$, and $U^{(3)}$ satisfying the conditions above. Moreover, in view of Definitions 8.2 and 9.2, the Lie derivatives $\mathscr{L}_{U^{(1)}}(g), \mathscr{L}_{U^{(2)}}(g)$, and $\mathscr{L}_{U^{(3)}}(g)$ are small in the $C^{2}$-norm. Consequently, the vector fields $U^{(1)}, U^{(2)}$, and $U^{(3)}$ are close to the standard rotation vector fields on the Bryant soliton in the $C^{2,1 / 2}$-norm.

Step 2. Suppose next that $\bar{t}=t_{k}$. In this case, the assertion follows from the result in Step 1 by passing to the limit. Since the vector fields constructed in Step 1 are bounded in $C^{2,1 / 2}$, we may take the limit in $C^{2}$.

LEMMA 9.18. If $j$ is sufficiently large and $k$ is sufficiently large depending on $j$, then the following statement holds. Consider a time $\bar{t} \in\left[t_{k}-2^{j / 100} r_{k}^{2}, t_{k}\right]$. Suppose that $U^{(1)}$, $U^{(2)}$, and $U^{(3)}$ are vector fields on $B_{g(\bar{t})}\left(p_{k}, 2^{j / 400} \Lambda r_{k}\right)$ with the following properties:

$$
\left|\mathscr{L}_{U^{(a)}}(g(\bar{t}))\right|+r_{k}\left|D\left(\mathscr{L}_{U^{(a)}}(g(\bar{t}))\right)\right| \leqslant C\left(r_{k}^{-1} d_{g(\bar{t})}\left(p_{k}, x\right)+1\right)^{-100} \varepsilon_{k}
$$

for all $x \in B_{g(\bar{t})}\left(p_{k}, 2^{j / 400} \Lambda r_{k}\right)$;

- if $x \in B_{g(\bar{t})}\left(p_{k}, 2^{j / 400} \Lambda r_{k}\right) \backslash B_{g(\bar{t})}\left(p_{k}, 4 \Lambda r_{k}\right)$, then

$$
r_{k}^{-1}\left|\left\langle U^{(a)}, \nu\right\rangle\right| \leqslant C\left(r_{k}^{-1} d_{g(\bar{t})}\left(p_{k}, x\right)+1\right)^{-100} \varepsilon_{k},
$$

where $\nu$ denotes the unit normal to the $C M C$ foliation of $(M, g(\bar{t}))$;

- if $x \in B_{g(\bar{t})}\left(p_{k}, 2^{j / 400} \Lambda r_{k}\right) \backslash B_{g(\bar{t})}\left(p_{k}, 4 \Lambda r_{k}\right)$, then

$$
\sum_{a, b=1}^{3}\left|\delta_{a b}-\operatorname{area}_{g(\bar{t})}(\Sigma)^{-2} \int_{\Sigma}\left\langle U^{(a)}, U^{(b)}\right\rangle_{g(\bar{t})} d \mu_{g(\bar{t})}\right| \leqslant C\left(r_{k}^{-1} d_{g(\bar{t})}\left(p_{k}, x\right)+1\right)^{-100} \varepsilon_{k},
$$

where $\Sigma$ denotes the leaf of the CMC foliation passing through $(x, \bar{t})$. 
Moreover, suppose that $\widetilde{U}^{(1)}, \widetilde{U}^{(2)}$, and $\widetilde{U}^{(3)}$ are vector fields on $B_{g(\bar{t})}\left(p_{k}, 2^{j / 400} \Lambda r_{k}\right)$ with the following properties:

$$
\left|\mathscr{L}_{\widetilde{U}^{(a)}}(g(\bar{t}))\right|+r_{k}\left|D\left(\mathscr{L}_{\widetilde{U}^{(a)}}(g(\bar{t}))\right)\right| \leqslant C\left(r_{k}^{-1} d_{g(\bar{t})}\left(p_{k}, x\right)+1\right)^{-100} \varepsilon_{k}
$$

for all $x \in B_{g(\bar{t})}\left(p_{k}, 2^{j / 400} \Lambda r_{k}\right)$;

- if $x \in B_{g(\bar{t})}\left(p_{k}, 2^{j / 400} \Lambda r_{k}\right) \backslash B_{g(\bar{t})}\left(p_{k}, 4 \Lambda r_{k}\right)$, then

$$
r_{k}^{-1}\left|\left\langle\widetilde{U}^{(a)}, \nu\right\rangle\right| \leqslant C\left(r_{k}^{-1} d_{g(\bar{t})}\left(p_{k}, x\right)+1\right)^{-100} \varepsilon_{k},
$$

where $\nu$ denotes the unit normal to the CMC foliation of $(M, g(\bar{t}))$;

- if $x \in B_{g(\bar{t})}\left(p_{k}, 2^{j / 400} \Lambda r_{k}\right) \backslash B_{g(\bar{t})}\left(p_{k}, 4 \Lambda r_{k}\right)$, then

$$
\sum_{a, b=1}^{3}\left|\delta_{a b}-\operatorname{area}_{g(\bar{t})}(\Sigma)^{-2} \int_{\Sigma}\left\langle\widetilde{U}^{(a)}, \widetilde{U}^{(b)}\right\rangle_{g(\bar{t})} d \mu_{g(\bar{t})}\right| \leqslant C\left(r_{k}^{-1} d_{g(\bar{t})}\left(p_{k}, x\right)+1\right)^{-100} \varepsilon_{k},
$$

where $\Sigma$ denotes the leaf of the CMC foliation passing through $(x, \bar{t})$.

Then there exists a $3 \times 3$ matrix $\omega \in O(3)$ such that

$$
r_{k}^{-1} \sum_{a=1}^{3}\left|\sum_{b=1}^{3} \omega_{a b} U^{(b)}-\widetilde{U}^{(a)}\right|_{g(\bar{t})} \leqslant C\left(r_{k}^{-1} d_{g(\bar{t})}\left(p_{k}, x\right)+1\right)^{-20} \varepsilon_{k}
$$

on $B_{g(\bar{t})}\left(p_{k}, 2^{(j-1) / 400} \Lambda r_{k}\right)$.

Proof. For each integer $m \in\left[8 \Lambda, 2^{(j-1) / 400} \Lambda\right]$, Proposition 7.4 implies that there exists a $3 \times 3$ matrix $\omega^{(m)} \in O(3)$ such that

$$
r_{k}^{-1} \sum_{a=1}^{3}\left|\sum_{b=1}^{3} \omega_{a b}^{(m)} U^{(b)}-\widetilde{U}^{(a)}\right|_{g(\bar{t})} \leqslant C m^{-80} \varepsilon_{k}
$$

on $B_{g(\bar{t})}\left(p_{k},(m+1) r_{k}\right) \backslash B_{g(\bar{t})}\left(p_{k},(m-1) r_{k}\right)$. Note that $\left|\omega^{(m)}-\omega^{(m+1)}\right| \leqslant C m^{-60} \varepsilon_{k}$. Consequently, there exists a $3 \times 3$ matrix $\omega \in O(3)$ such that $\left|\omega^{(m)}-\omega\right| \leqslant C m^{-40} \varepsilon_{k}$. Hence, for every integer $m \in\left[8 \Lambda, 2^{(j-1) / 400} \Lambda\right]$, we obtain

$$
r_{k}^{-1} \sum_{a=1}^{3}\left|\sum_{b=1}^{3} \omega_{a b} U^{(b)}-\widetilde{U}^{(a)}\right|_{g(\bar{t})} \leqslant C m^{-20} \varepsilon_{k}
$$

on $B_{g(\bar{t})}\left(p_{k},(m+1) r_{k}\right) \backslash B_{g(\bar{t})}\left(p_{k},(m-1) r_{k}\right)$. Using Lemma 7.1, we deduce that

$$
r_{k}^{-1} \sum_{a=1}^{3}\left|\sum_{b=1}^{3} \omega_{a b} U^{(b)}-\widetilde{U}^{(a)}\right|_{g(\bar{t})} \leqslant C \varepsilon_{k}
$$

on $B_{g(\bar{t})}\left(p_{k}, 16 \Lambda r_{k}\right)$. 
In the following, we define

$$
\Omega^{(j, k)}:=\left\{(x, t) \in B_{g\left(t_{k}\right)}\left(p_{k}, \delta_{k}^{-1} r_{k}\right) \times\left[t_{k}-2^{j / 100} r_{k}^{2}, t_{k}\right]: f(x, t) \leqslant 2^{j / 400}\right\}
$$

where $f: B_{g\left(t_{k}\right)}\left(p_{k}, \delta_{k}^{-1} r_{k}\right) \times\left[t_{k}-\delta_{k}^{-1} r_{k}^{2}, t_{k}\right] \rightarrow \mathbb{R}$ is the function in Corollary 9.12. We now state the main result of this section.

Proposition 9.19. Let $j$ be a large positive integer. If $k$ is sufficiently large (depending on $j$ ), then we can find time-independent vector fields $W^{(1)}, W^{(2)}$, and $W^{(3)}$ such that

$$
\sum_{l=0}^{40} r_{k}^{l}\left|D^{l}\left(\mathscr{L}_{W^{(a)}}(g)\right)\right| \leqslant C 2^{-j / 400} \varepsilon_{k}
$$

for all points $(x, t) \in B_{g\left(t_{k}\right)}\left(p_{k}, 4 \Lambda r_{k}\right) \times\left[t_{k}-1000 K \Lambda r_{k}^{2}, t_{k}\right]$. Here, $C$ is a constant which is independent of $j$ and $k$. Finally, on the set $B_{g\left(t_{k}\right)}\left(p_{k}, 4 \Lambda r_{k}\right) \times\left[t_{k}-1000 K \Lambda r_{k}^{2}, t_{k}\right]$, the vector fields $W^{(1)}, W^{(2)}$, and $W^{(3)}$ are close to the standard rotation vector fields on the Bryant soliton in the $C^{80}$-norm.

Proof. We will assume throughout that $j$ is large, and $k$ is sufficiently large depending on $j$. This ensures, after rescaling by the factor $r_{k}^{-1}$, the domain $\Omega^{(j, k)}$ is close to a piece of the Bryant soliton. By Corollary 9.12, the function $f: \Omega^{(j, k)} \rightarrow \mathbb{R}$ satisfies $R-\Delta f \leqslant 3 \delta_{k} r_{k}^{-2}$ and $\Delta f+|\nabla f|^{2} \leqslant\left(1+\delta_{k}\right) r_{k}^{-2}$, and hence

$$
R+|\nabla f|^{2} \leqslant\left(1+4 \delta_{k}\right) r_{k}^{-2} \leqslant 2 r_{k}^{-2}
$$

Moreover, Corollary 9.12 implies that

$$
\Delta f+|\nabla f|^{2} \geqslant\left(1-\delta_{k}\right) r_{k}^{-2} \text { and } \quad \frac{\partial f}{\partial t}+|\nabla f|^{2} \leqslant \delta_{k} r_{k}^{-2}
$$

and hence

$$
\frac{\partial}{\partial t} f-\Delta f \leqslant-\left(1-2 \delta_{k}\right) r_{k}^{-2} \leqslant-\frac{1}{2} r_{k}^{-2} .
$$

Note that

$$
\frac{1}{2 K}\left(r_{k}^{-1} d_{g(t)}\left(p_{k}, x\right)+1\right) \leqslant f(x, t)+1 \leqslant 2 K\left(r_{k}^{-1} d_{g(t)}\left(p_{k}, x\right)+1\right)
$$

by Corollary 9.12 .

Step 1. Using Lemmas 9.17 and 9.18, we can construct time-dependent vector fields $U^{(1)}, U^{(2)}$, and $U^{(3)}$, defined on $\Omega^{(j, k)}$, with the following properties:

- $r_{k}\left|\partial U^{(a)} / \partial t\right| \leqslant C(f+100)^{-10} \varepsilon_{k}$ on $\Omega^{(j, k)}$;

- $\left|\mathscr{L}_{U^{(a)}}(g)\right|+r_{k}\left|D\left(\mathscr{L}_{U^{(a)}}(g)\right)\right| \leqslant C(f+100)^{-100} \varepsilon_{k}$ on $\Omega^{(j, k)}$. 
Here, $C$ is a large constant that does not depend on $j$ or $k$. Moreover, we can arrange that, on the set $\Omega^{(j, k)}$, the vector fields $U^{(1)}, U^{(2)}$, and $U^{(3)}$ are close to the standard rotation vector fields on the Bryant soliton in the $C^{2}$-norm. Note that

$$
r_{k}\left|\Delta U^{(a)}+\operatorname{Ric}\left(U^{(a)}\right)\right| \leqslant C r_{k}\left|D\left(\mathscr{L}_{U^{(a)}}(g)\right)\right| \leqslant C(f+100)^{-100} \varepsilon_{k} \quad \text { on } \Omega^{(j, k)} .
$$

Step 2. Let $V^{(a)}$ denote the solution of the PDE

$$
\frac{\partial}{\partial t} V^{(a)}=\Delta V^{(a)}+\operatorname{Ric}\left(V^{(a)}\right) \quad \text { on } \Omega^{(j, k)}
$$

with Dirichlet boundary condition $V^{(a)}=U^{(a)}$ on the parabolic boundary of $\Omega^{(j, k)}$. Using the estimate

$$
r_{k}\left|\frac{\partial}{\partial t} U^{(a)}-\Delta U^{(a)}-\operatorname{Ric}\left(U^{(a)}\right)\right| \leqslant C(f+100)^{-10} \varepsilon_{k},
$$

we obtain

$$
r_{k}\left|\frac{\partial}{\partial t}\left(V^{(a)}-U^{(a)}\right)-\Delta\left(V^{(a)}-U^{(a)}\right)-\operatorname{Ric}\left(V^{(a)}-U^{(a)}\right)\right| \leqslant C(f+100)^{-10} \varepsilon_{k}
$$

in $\Omega^{(j, k)}$, where $C$ is a large constant that does not depend on $j$ or $k$. Proposition 5.3 gives

$$
r_{k}\left(\frac{\partial}{\partial t}-\Delta\right)\left|V^{(a)}-U^{(a)}\right| \leqslant C(f+100)^{-10} \varepsilon_{k}
$$

in $\Omega^{(j, k)}$, where $C$ is a large constant that does not depend on $j$ or $k$.

Using the inequalities

$$
\left(\frac{\partial}{\partial t}-\Delta\right) f \leqslant-\frac{1}{2} r_{k}^{-2} \text { and }|\nabla f|^{2} \leqslant 2 r_{k}^{-2},
$$

we obtain

$$
\begin{aligned}
\left(\frac{\partial}{\partial t}-\Delta\right)(f+100)^{-8} & =-8(f+100)^{-9}\left(\frac{\partial}{\partial t}-\Delta\right) f-72(f+100)^{-10}|\nabla f|^{2} \\
& \geqslant 4(f+100)^{-9} r_{k}^{-2}-144(f+100)^{-10} r_{k}^{-2} \\
& \geqslant(f+100)^{-9} r_{k}^{-2}
\end{aligned}
$$

in $\Omega^{(j, k)}$. Using the maximum principle, we conclude that

$$
r_{k}^{-1}\left|V^{(a)}-U^{(a)}\right| \leqslant C(f+100)^{-8} \varepsilon_{k}
$$

in $\Omega^{(j, k)}$, where $C$ is a large constant that does not depend on $j$ or $k$. Using standard interior estimates for linear parabolic equations, we obtain

$$
\left|D\left(V^{(a)}-U^{(a)}\right)\right| \leqslant C(f+100)^{-8} \varepsilon_{k}
$$


in $\Omega^{(j-1, k)}$, where $C$ is a large constant that does not depend on $j$ or $k$. In particular, on the set $\Omega^{(j-1, k)}$, the vector fields $V^{(1)}, V^{(2)}$, and $V^{(3)}$ are close to the standard rotation vector fields on the Bryant soliton in the $C^{1}$-norm. Consequently, on the set

$$
B_{g\left(t_{k}\right)}\left(p_{k}, 8 \Lambda r_{k}\right) \times\left[t_{k}-2000 K \Lambda r_{k}^{2}, t_{k}\right],
$$

the vector fields $V^{(1)}, V^{(2)}$, and $V^{(3)}$ are close to the standard rotation vector fields on the Bryant soliton in the $C^{100}$-norm.

Step 3 . We now define $h^{(a)}(t):=\mathscr{L}_{V^{(a)}(t)}(g(t))$. Since

$$
\frac{\partial}{\partial t} V^{(a)}=\Delta V^{(a)}+\operatorname{Ric}\left(V^{(a)}\right)
$$

we obtain

$$
\frac{\partial}{\partial t} h^{(a)}(t)=\Delta_{L, g(t)} h^{(a)}(t)
$$

by Corollary 5.2. The estimate for $V^{(a)}-U^{(a)}$ in Step 2 implies that

$$
\left|h^{(a)}\right| \leqslant\left|\mathscr{L}_{U^{(a)}}(g)\right|+C\left|D\left(V^{(a)}-U^{(a)}\right)\right| \leqslant C(f+100)^{-8} \varepsilon_{k}
$$

in $\Omega^{(j-1, k)}$, where $C$ is a large constant that does not depend on $j$ or $k$.

Let $C_{\#}$ and $c_{\#}$ denote the constants in Theorem B.1. If $j$ is sufficiently large and $k$ is sufficiently large depending on $j$, then $C_{\#} 2^{-j / 200} r_{k}^{-2} \leqslant R(x, t) \leqslant r_{k}^{-2}$ for all points $(x, t) \in \Omega^{(j, k)}$. Therefore, we may apply Theorem B.1 with $\varrho:=2^{-j / 200} r_{k}^{-2}$. Consequently, the function

$$
\psi^{(a)}:=\exp \left(-c_{\#} 2^{-j / 200} r_{k}^{-2}\left(t_{k}-t\right)\right) \frac{\left|h^{(a)}\right|}{r_{k}^{2} R-2^{-j / 200}}
$$

satisfies

$$
\left(\frac{\partial}{\partial t}-\Delta-\frac{2 r_{k}^{2}}{r_{k}^{2} R-2^{-j / 200}} D^{i} R D_{i}\right)\left(\psi^{(a)}\right)^{2} \leqslant 0
$$

on the set $\Omega^{(j-1, k)}$. We now apply the maximum principle to the function $\psi^{(a)}$ on the set $\Omega^{(j-1, k)}$. This gives

$$
\psi^{(a)} \leqslant C 2^{-j / 400} \varepsilon_{k}
$$

on the set $\Omega^{(j-1, k)}$, where $C$ is a large constant that does not depend on $j$ or $k$. Since $r_{k}^{2} R(x, t) \leqslant 1$ for all $t \leqslant t_{k}$, it follows that

$$
\left|h^{(a)}\right| \leqslant C 2^{-j / 400} \varepsilon_{k}
$$

on the set $B_{g\left(t_{k}\right)}\left(p_{k}, 16 \Lambda r_{k}\right) \times\left[t_{k}-2000 K \Lambda r_{k}^{2}, t_{k}\right]$, where $C$ is a large constant that does not depend on $j$ or $k$. Using standard interior estimates for linear parabolic equations, we obtain

$$
\sum_{l=0}^{100} r_{k}^{l}\left|D^{l} h^{(a)}\right| \leqslant C 2^{-j / 400} \varepsilon_{k}
$$


on the set $B_{g\left(t_{k}\right)}\left(p_{k}, 8 \Lambda r_{k}\right) \times\left[t_{k}-1000 K \Lambda r_{k}^{2}, t_{k}\right]$. To summarize, we have shown that

$$
\sum_{l=0}^{100} r_{k}^{l}\left|D^{l}\left(\mathscr{L}_{V^{(a)}}(g)\right)\right| \leqslant C 2^{-j / 400} \varepsilon_{k}
$$

on the set $B_{g\left(t_{k}\right)}\left(p_{k}, 8 \Lambda r_{k}\right) \times\left[t_{k}-1000 K \Lambda r_{k}^{2}, t_{k}\right]$. Moreover, using the identity

$$
\frac{\partial}{\partial t} V^{(a)}=\Delta V^{(a)}+\operatorname{Ric}\left(V^{(a)}\right)=\operatorname{div} h^{(a)}-\frac{1}{2} \nabla\left(\operatorname{tr} h^{(a)}\right),
$$

we obtain

$$
\sum_{l=0}^{80} r_{k}^{l+1}\left|D^{l}\left(\frac{\partial}{\partial t} V^{(a)}\right)\right| \leqslant C 2^{-j / 400} \varepsilon_{k},
$$

on the set $B_{g\left(t_{k}\right)}\left(p_{k}, 8 \Lambda r_{k}\right) \times\left[t_{k}-1000 K \Lambda r_{k}^{2}, t_{k}\right]$.

Step 4. Let $W^{(1)}, W^{(2)}$, and $W^{(3)}$ be time-independent vector fields such that $W^{(a)}=V^{(a)}$ at time $t_{k}$. On the set $B_{g\left(t_{k}\right)}\left(p_{k}, 8 \Lambda r_{k}\right) \times\left[t_{k}-1000 K \Lambda r_{k}^{2}, t_{k}\right]$, the vector fields $W^{(1)}, W^{(2)}$, and $W^{(3)}$ are close to the standard rotation vector fields on the Bryant soliton. Using the estimate for $\partial V^{(a)} / \partial t$ in Step 3, we obtain

$$
\sum_{l=0}^{60} r_{k}^{l-1}\left|D^{l}\left(W^{(a)}-V^{(a)}\right)\right| \leqslant C 2^{-j / 400} \varepsilon_{k},
$$

and hence

$$
\sum_{l=0}^{40} r_{k}^{l}\left|D^{l}\left(\mathscr{L}_{W^{(a)}}(g)\right)\right| \leqslant C 2^{-j / 400} \varepsilon_{k}
$$

on the set $B_{g\left(t_{k}\right)}\left(p_{k}, 8 \Lambda r_{k}\right) \times\left[t_{k}-1000 K \Lambda r_{k}^{2}, t_{k}\right]$.

For each $k$ large, we choose a compact domain $D_{k} \subset M$ with the following properties:

- there exists a point $x \in \partial D_{k}$ such that $\lambda_{1}\left(x, t_{k}\right)=\frac{2}{3} \theta R\left(x, t_{k}\right)$;

- for each $x \in D_{k}$, we have $\lambda_{1}\left(x, t_{k}\right) \geqslant \frac{2}{3} \theta R\left(x, t_{k}\right)$;

- $\partial D_{k}$ is a leaf of the CMC foliation of $\left(M, g\left(t_{k}\right)\right)$.

Note that

$$
D_{k} \subset\left\{x \in M: \lambda_{1}\left(x, t_{k}\right)>\frac{1}{2} \theta R\left(x, t_{k}\right)\right\} \subset B_{g\left(t_{k}\right)}\left(p_{k}, \Lambda r_{k}\right),
$$

in view of our choice of $\Lambda$. Moreover, if $\bar{x} \in M \backslash D_{k}$, then the point $\left(\bar{x}, t_{k}\right)$ lies at the center of an evolving $\varepsilon_{1}$-neck by Proposition 9.1. 
Proposition 9.20. Let $j$ be a large positive integer. If $k$ is sufficiently large (depending on $j$ ), then the vector fields $W^{(1)}, W^{(2)}$, and $W^{(3)}$ constructed in Proposition 9.19 have the following property. For each point $\bar{x} \in B_{g\left(t_{k}\right)}\left(p_{k}, \Lambda r_{k}\right) \backslash D_{k}$, we have

$$
\sum_{l=0}^{10} r_{k}^{l-1}\left|D^{l}\left(\left\langle W^{(a)}, \nu\right\rangle\right)\right| \leqslant C 2^{-j / 400} \varepsilon_{k}
$$

on the parabolic neighborhood

$$
B_{g\left(t_{k}\right)}\left(\bar{x}, 600 R\left(\bar{x}, t_{k}\right)^{-1 / 2}\right) \times\left[t_{k}-200 R\left(\bar{x}, t_{k}\right)^{-1}, t_{k}\right] .
$$

Here, $\nu$ denotes the unit normal to the CMC foliation and $C$ is a constant which is independent of $j$ and $k$.

Proof. Let us consider a point $\bar{x} \in B_{g\left(t_{k}\right)}\left(p_{k}, \Lambda r_{k}\right) \backslash D_{k}$. Recall that the point $\left(\bar{x}, t_{k}\right)$ lies at the center of an evolving $\varepsilon_{1}$-neck. By Corollary $9.12, R\left(\bar{x}, t_{k}\right)^{-1} \leqslant 4 K \Lambda r_{k}^{2}$. Since $\sqrt{4 K / \Lambda} \leqslant 10^{-6}$, we obtain $R\left(\bar{x}, t_{k}\right)^{-1 / 2} \leqslant 10^{-6} \Lambda r_{k}$. Hence, the parabolic neighborhood

$$
B_{g\left(t_{k}\right)}\left(\bar{x}, 1000 R\left(\bar{x}, t_{k}\right)^{-1 / 2}\right) \times\left[t_{k}-200 R\left(\bar{x}, t_{k}\right)^{-1}, t_{k}\right]
$$

is contained in

$$
B_{g\left(t_{k}\right)}\left(p_{k}, 4 \Lambda r_{k}\right) \times\left[t_{k}-1000 K \Lambda r_{k}^{2}, t_{k}\right]
$$

In particular, the estimates in Proposition 9.19 hold on the parabolic neighborhood

$$
B_{g\left(t_{k}\right)}\left(\bar{x}, 1000 R\left(\bar{x}, t_{k}\right)^{-1 / 2}\right) \times\left[t_{k}-200 R\left(\bar{x}, t_{k}\right)^{-1}, t_{k}\right]
$$

Let us fix a time $t \in\left[t_{k}-200 R\left(\bar{x}, t_{k}\right)^{-1}, t_{k}\right]$, and let $\Sigma_{s}$ denote the CMC foliation of $(M, g(t))$. Note that the foliation depends on $t$, but we suppress this dependence in our notation. In the following, we only consider those leaves of the foliation that are contained in $B_{g\left(t_{k}\right)}\left(\bar{x}, 800 R\left(\bar{x}, t_{k}\right)^{-1 / 2}\right)$. We define a function $F^{(a)}: \Sigma_{s} \rightarrow \mathbb{R}$ by $F^{(a)}:=\left\langle W^{(a)}, \nu\right\rangle$. The quantity

$$
\Delta_{\Sigma_{s}} F^{(a)}+\left(|A|^{2}+\operatorname{Ric}(\nu, \nu)\right) F^{(a)}=: H^{(a)}
$$

can be expressed in terms of $\mathscr{L}_{W^{(a)}}(g)$ and the first derivatives of $\mathscr{L}_{W^{(a)}}(g)$. Using the estimate for $\mathscr{L}_{W^{(a)}}(g)$ in Step 4 , we obtain

$$
\sum_{l=0}^{20} r_{k}^{l+1}\left|D^{l} H^{(a)}\right| \leqslant C 2^{-j / 400} \varepsilon_{k} .
$$

We now consider the spectrum of the Jacobi operator $\Delta_{\Sigma_{s}}+\left(|A|^{2}+\operatorname{Ric}(\nu, \nu)\right)$ on $\Sigma_{s}$. Since $\Sigma_{s} \subset B_{g\left(t_{k}\right)}\left(\bar{x}, 800 R\left(\bar{x}, t_{k}\right)^{-1 / 2}\right) \subset B_{g\left(t_{k}\right)}\left(p_{k}, 4 \Lambda r_{k}\right)$, the eigenvalues of the Jacobi operator lie outside the interval $\left[-c r_{k}^{-2}, c r_{k}^{-2}\right]$ for some small positive constant $c$ which is 
independent of $j$ and $k$. (This can be easily verified on the Bryant soliton. For the general case, we observe that the actual solution is a small perturbation of the Bryant soliton in the relevant region.) Consequently, we can invert the Jacobi operator. Using the estimate

$$
\sum_{l=0}^{20} r_{k}^{l+1}\left|D^{l} H^{(a)}\right| \leqslant C 2^{-j / 400} \varepsilon_{k}
$$

we obtain

$$
\sum_{l=0}^{10} r_{k}^{l-1}\left|D^{l} F^{(a)}\right| \leqslant C 2^{-j / 400} \varepsilon_{k} .
$$

Since $t \in\left[t_{k}-200 R\left(\bar{x}, t_{k}\right)^{-1}, t_{k}\right]$ is arbitrary, we conclude that

$$
\sum_{l=0}^{10} r_{k}^{l-1}\left|D^{l}\left(\left\langle W^{(a)}, \nu\right\rangle\right)\right| \leqslant C 2^{-j / 400} \varepsilon_{k}
$$

on $B_{g\left(t_{k}\right)}\left(\bar{x}, 600 R\left(\bar{x}, t_{k}\right)^{-1 / 2}\right) \times\left[t_{k}-200 R\left(\bar{x}, t_{k}\right)^{-1}, t_{k}\right]$.

Proposition 9.21. Let $j$ be a large integer. If $k$ is sufficiently large (depending on $j)$, then the vector fields $W^{(1)}, W^{(2)}$, and $W^{(3)}$ constructed in Proposition 9.19 have the following property. For each point $\bar{x} \in B_{g\left(t_{k}\right)}\left(p_{k}, \Lambda r_{k}\right) \backslash D_{k}$, we can find a symmetric $3 \times 3$ matrix $Q_{a b}$ such that

$$
\left|Q_{a b}-\operatorname{area}_{g(t)}(\Sigma)^{-2} \int_{\Sigma}\left\langle W^{(a)}, W^{(b)}\right\rangle_{g(t)} d \mu_{g(t)}\right| \leqslant C 2^{-j / 400} \varepsilon_{k}
$$

whenever $t \in\left[t_{k}-200 R\left(\bar{x}, t_{k}\right)^{-1}, t_{k}\right]$ and $\Sigma \subset B_{g\left(t_{k}\right)}\left(\bar{x}, 200 R\left(\bar{x}, t_{k}\right)^{-1 / 2}\right)$ is a leaf of the $C M C$ foliation of $(M, g(t))$. Note that $Q_{a b}$ is independent of $t$ and $\Sigma$. Moreover, the eigenvalues of the matrix $Q_{a b}$ lie in the interval $[1 / C, C]$, where $C$ is independent of $j$ and $k$.

Proof. Let us consider a point $\bar{x} \in B_{g\left(t_{k}\right)}\left(p_{k}, \Lambda r_{k}\right) \backslash D_{k}$. Recall that the point $\left(\bar{x}, t_{k}\right)$ lies at the center of an evolving $\varepsilon_{1}$-neck. Moreover, since

$$
R\left(\bar{x}, t_{k}\right)^{-1} \leqslant 4 K \Lambda r_{k}^{2} \quad \text { and } \quad R\left(\bar{x}, t_{k}\right)^{-1 / 2} \leqslant 10^{-6} \Lambda r_{k},
$$

the parabolic neighborhood

$$
B_{g\left(t_{k}\right)}\left(\bar{x}, 600 R\left(\bar{x}, t_{k}\right)^{-1 / 2}\right) \times\left[t_{k}-200 R\left(\bar{x}, t_{k}\right)^{-1}, t_{k}\right]
$$

is contained in

$$
B_{g\left(t_{k}\right)}\left(p_{k}, 4 \Lambda r_{k}\right) \times\left[t_{k}-1000 K \Lambda r_{k}^{2}, t_{k}\right] .
$$


Therefore, the estimates in Propositions 9.19 and 9.20 hold on the parabolic neighborhood

$$
B_{g\left(t_{k}\right)}\left(\bar{x}, 600 R\left(\bar{x}, t_{k}\right)^{-1 / 2}\right) \times\left[t_{k}-200 R\left(\bar{x}, t_{k}\right)^{-1}, t_{k}\right]
$$

We now argue as in Steps 11-14 in the proof of the neck improvement theorem. This implies that there exists a symmetric $3 \times 3$ matrix $Q_{a b}$ such that

$$
\left|Q_{a b}-\operatorname{area}_{g(t)}(\Sigma)^{-2} \int_{\Sigma}\left\langle W^{(a)}, W^{(b)}\right\rangle_{g(t)} d \mu_{g(t)}\right| \leqslant C 2^{-j / 400} \varepsilon_{k}
$$

whenever $t \in\left[t_{k}-200 R\left(\bar{x}, t_{k}\right)^{-1}, t_{k}\right]$ and $\Sigma \subset B_{g\left(t_{k}\right)}\left(\bar{x}, 200 R\left(\bar{x}, t_{k}\right)^{-1 / 2}\right)$ is a leaf of the CMC foliation of $(M, g(t))$. Finally, since the vector fields $W^{(1)}, W^{(2)}$, and $W^{(3)}$ are close to the standard rotation vector fields on the Bryant soliton, the eigenvalues of the matrix $Q_{a b}$ are uniformly bounded from above and below.

COROLlary 9.22. If $k$ is sufficiently large, then $\left(\bar{x}, t_{k}\right)$ is $\frac{1}{2} \varepsilon_{k}$-symmetric for all $\bar{x} \in B_{g\left(t_{k}\right)}\left(p_{k}, \Lambda r_{k}\right) \backslash D_{k}$.

Proof. This follows by combining Propositions 9.19-9.21.

We can now complete the proof of Theorem 1.2. Combining Lemma 9.15 and Corollary 9.22 , we conclude that $\left(\bar{x}, t_{k}\right)$ is $\frac{1}{2} \varepsilon_{k}$-symmetric for all $\bar{x} \in M \backslash D_{k}$. Moreover, if $k$ is sufficiently large, it follows from Propositions 9.19-9.21 that there exist vector fields on the cap $D_{k}$ which satisfy the requirements of Definition 9.2 with $\varepsilon=\frac{1}{2} \varepsilon_{k}$. Therefore, the flow is $\frac{1}{2} \varepsilon_{k}$-symmetric at time $t_{k}$ if $k$ is sufficiently large. By Lemma 9.5 , we can find a time $\tilde{t}_{k}>t_{k}$ with the property that the flow is $\varepsilon_{k}$-symmetric at time $t$ for all $t \in\left[t_{k}, \tilde{t}_{k}\right]$. This contradicts the definition of $t_{k}$. This completes the proof of Theorem 1.2.

\section{Appendix A. Summary of known results about ancient $\kappa$-solutions}

In this appendix, we collect some of the main known results on ancient $\kappa$-solutions, which we use in this paper. We first recall a basic Riemannian geometry fact.

Proposition A.1. Let $(M, g)$ be a complete, non-compact manifold with positive sectional curvature, and let $N$ be a neck in $M$. Let $U$ denote the unbounded connected component of $M \backslash N$. If every point in $N \cup U$ lies at the center of a neck, then

$$
\sup _{U} R \leqslant C \sup _{N} R
$$

Proof. The assertion is a consequence of [20, Corollary 2.21]. (Note that the soul cannot lie at the center of a neck, and therefore must be contained in $M \backslash(N \cup U)$.) 
In the following, we give an alternative argument for the convenience of the reader. By assumption, every point in $N \cup U$ lies at the center of a neck. Hence, by work of Hamilton, there is a canonical CMC foliation $\Sigma_{s}, s \in[0, \infty)$, such that

$$
\Sigma_{0} \subset N \quad \text { and } \quad U \subset \bigcup_{s \in[0, \infty)} \Sigma_{s} \subset N \cup U .
$$

Let $v$ denote the lapse function of this CMC foliation. We assume that the lapse function $v$ has mean value 1 , so that $\int_{\Sigma_{s}} v=\operatorname{area}\left(\Sigma_{s}\right)$ for each $s$. Note that $\sup _{\Sigma_{s}}|v-1|$ is very small; in particular, $v$ is positive. We next compute

$$
-\frac{d}{d s} H=\Delta_{\Sigma_{s}} v+\left(|A|^{2}+\operatorname{Ric}(\nu, \nu)\right) v \geqslant \Delta_{\Sigma_{s}} v+\frac{1}{2} H^{2} v
$$

at each point on $\Sigma_{s}$. We now take the mean value over $\Sigma_{s}$. Clearly, $\Delta_{\Sigma_{s}} v$ has mean value 0 , by the divergence theorem. Moreover, since $H$ is constant on $\Sigma_{s}$ and $v$ has mean value 1 , it follows that the function $H^{2} v$ has mean value $H^{2}$. This gives

$$
-\frac{d}{d s} H \geqslant \frac{1}{2} H^{2}
$$

Hence, if $H(s)<0$ for some $s$, then $H(s)$ converges to $-\infty$ at a finite value of $s$, which is impossible. Therefore, $H(s) \geqslant 0$ for all $s$. Consequently, area $\left(\Sigma_{s}\right)$ is an increasing function of $s$. This implies that

$$
\frac{1}{C} \sup _{\Sigma_{s}} R \leqslant \operatorname{area}\left(\Sigma_{s}\right)^{-1} \leqslant \operatorname{area}\left(\Sigma_{0}\right)^{-1} \leqslant C \sup _{\Sigma_{0}} R
$$

for all $s \in[0, \infty)$. From this, the assertion follows.

We now recall the following fundamental theorem due to Perelman.

Theorem A.2. (Perelman $[21, \S 11.8])$ Let $(M, g(t))$ be a 3 -dimensional ancient $\kappa$ solution which is non-compact and has positive sectional curvature. Given any $\varepsilon>0$, we can find a compact domain $\Omega_{t} \subset M$ with the following properties:

- for each $x \in M \backslash \Omega_{t}$, the point $(x, t)$ lies at the center of an evolving $\varepsilon$-neck;

- the boundary $\partial \Omega_{t}$ is a leaf of the CMC foliation at time $t$;

- $\sup _{x \in \Omega_{t}} R(x, t) \leqslant C(\varepsilon) \inf _{x \in \Omega_{t}} R(x, t)$;

- $\operatorname{diam}_{g(t)}\left(\Omega_{t}\right)^{2} \sup _{x \in \Omega_{t}} R(x, t) \leqslant C(\varepsilon)$.

Combining Theorem A.2 with Proposition A.1 gives the following.

Corollary A.3. Let $(M, g(t))$ be a 3-dimensional ancient $\kappa$-solution which is noncompact and has positive sectional curvature. Let $\varepsilon$ be a small positive real number, and let $\Omega_{t}$ be as in Theorem A.2. Then,

$$
\sup _{x \in M} R(x, t) \leqslant C(\varepsilon) \inf _{x \in \Omega_{t}} R(x, t) \quad \text { and } \quad \operatorname{diam}_{g(t)}\left(\Omega_{t}\right)^{2} \sup _{x \in M} R(x, t) \leqslant C(\varepsilon) .
$$


Proof. Proposition A.1 implies

$$
\sup _{x \in M \backslash \Omega_{t}} R(x, t) \leqslant C \sup _{x \in \partial \Omega_{t}} R(x, t) .
$$

This gives

$$
\sup _{x \in M} R(x, t) \leqslant C \sup _{x \in \Omega_{t}} R(x, t) .
$$

Hence, the assertion follows from Theorem A.2.

Next result is a consequence of the neck stability theorem of Kleiner and Lott.

Theorem A.4. (Cf. Kleiner-Lott [18, Theorem 6.1]) Suppose that $(M, g(t))$ is a 3-dimensional ancient $\kappa$-solution which is non-compact and has positive sectional curvature. Then, there exists a point $q \in M$ such that $\sup _{t \leqslant 0}(-t) R(q, t) \leqslant 100$.

Proof. In the following, we give a proof for the convenience of the reader. Suppose that the assertion is false, so that $\sup _{t \leqslant 0}(-t) R(q, t)>100$ for each point $q \in M$. Let $q_{k}$ be a sequence of points going to infinity. For each $k$, we denote by $\ell_{k}(x, t)$ the reduced distance of $(x, t)$ from $\left(q_{k}, 0\right)$. Moreover, we denote by

$$
V_{k}(t)=(-t)^{-3 / 2} \int_{M} e^{-\ell_{k}(x, t)} d \operatorname{vol}_{g(t)}
$$

the reduced volume at time $t$.

By work of Perelman [21], we can find a sequence $\varepsilon_{k} \rightarrow 0$ such that the point $\left(q_{k}, 0\right)$ lies at the center of an evolving $\varepsilon_{k}$-neck (cf. Theorem A.2). This implies $(-t) R(x, t) \leqslant 10$ for all $t \in\left[-\varepsilon_{k}^{-1} R\left(q_{k}, 0\right)^{-1}, 0\right]$. Therefore, $\ell_{k}\left(q_{k}, t\right) \leqslant 100$ for all $t \in\left[-\varepsilon_{k}^{-1} R\left(q_{k}, 0\right)^{-1}, 0\right)$. By a result of Ye, there exists a universal constant $C$ such that

$$
\frac{d_{g(t)}(x, y)^{2}}{-t} \leqslant C\left(\ell_{k}(x, t)+\ell_{k}(y, t)+1\right)
$$

for all $t<0$ and all $x, y \in M$ (see [24], Lemma 3.2). Putting $y=q_{k}$ gives

$$
\frac{d_{g(t)}\left(x, q_{k}\right)^{2}}{-t} \leqslant C\left(\ell_{k}(x, t)+1\right)
$$

for all $t \in\left[-\varepsilon_{k}^{-1} R\left(q_{k}, 0\right)^{-1}, 0\right)$ and all $x \in M$.

Recall that the point $\left(q_{k}, 0\right)$ lies at the center of an evolving $\varepsilon_{k}$-neck. Using this fact together with Ye's estimate, we obtain

$$
\limsup _{k \rightarrow \infty} V_{k}\left(\tau R\left(q_{k}, 0\right)^{-1}\right) \leqslant V_{\text {cyl }}(\tau)
$$


for each $\tau<0$, where $V_{\text {cyl }}(\tau)$ denotes the reduced volume for a family of shrinking cylinders. Using the monotonicity of the reduced volume, we deduce that

$$
\limsup _{k \rightarrow \infty} V_{k}\left(-\varepsilon_{k}^{-1} R\left(q_{k}, 0\right)^{-1}\right) \leqslant V_{\text {cyl }}(\tau)
$$

for each $\tau<0$. Taking the limit as $\tau \rightarrow-\infty$ gives

$$
\limsup _{k \rightarrow \infty} V_{k}\left(-\varepsilon_{k}^{-1} R\left(q_{k}, 0\right)^{-1}\right) \leqslant V_{\text {cyl }}(-\infty)
$$

where $V_{\text {cyl }}(-\infty):=\lim _{\tau \rightarrow-\infty} V_{\text {cyl }}(\tau)$. On the other hand, since the asymptotic shrinking soliton is a family of shrinking cylinders, we have

$$
\lim _{t \rightarrow-\infty} V_{k}(t) \geqslant V_{\mathrm{cyl}}(-\infty)
$$

for each $k$. Since $V_{k}(t)$ is monotone increasing in $t$, it follows that

$$
V_{k}(t) \geqslant V_{\text {cyl }}(-\infty)
$$

for all $k$ and all $t$.

For each $k$, we define $t_{k}:=\sup \left\{t \leqslant 0:(-t) R\left(q_{k}, t\right)>10\right\}$. Clearly, $t_{k} \leqslant-\varepsilon_{k}^{-1} R\left(q_{k}, 0\right)^{-1}$, $\left(-t_{k}\right) R\left(q_{k}, t_{k}\right)=10$, and $(-t) R\left(q_{k}, t\right) \leqslant 10$ for all $t \in\left[t_{k}, 0\right]$. This implies

$$
\ell_{k}\left(q_{k}, t_{k}\right) \leqslant \frac{1}{2 \sqrt{-t_{k}}} \int_{t_{k}}^{0} \sqrt{-t} R\left(q_{k}, t\right) d t \leqslant 100
$$

The discussion above gives

$$
\inf _{t \in\left(-\infty, t_{k}\right]} V_{k}(t) \geqslant V_{\text {cyl }}(-\infty)
$$

and

$$
\sup _{t \in\left(-\infty, t_{k}\right]} V_{k}(t) \leqslant V_{k}\left(-\varepsilon_{k}^{-1} R\left(q_{k}, 0\right)^{-1}\right) \rightarrow V_{\text {cyl }}(-\infty)
$$

as $k \rightarrow \infty$. Hence, if we dilate the flow $(M, g(t))$ around $\left(q_{k}, t_{k}\right)$ by the factor $\left(-t_{k}\right)^{-1 / 2}$, then the rescaled flows converge in the Cheeger-Gromov sense to a shrinking gradient Ricci soliton (see [21, §11]). Since $\left(-t_{k}\right) R\left(q_{k}, t_{k}\right)=10$ for each $k$, this limiting soliton is non-flat, and consequently must be a cylinder with scalar curvature 1 (cf. $[22, \S 1])$. In particular, $\left(-t_{k}\right) R\left(q_{k}, t_{k}\right) \rightarrow 1$ as $k \rightarrow \infty$. This contradicts the fact that $\left(-t_{k}\right) R\left(q_{k}, t_{k}\right)=10$ for each $k$. 


\section{Appendix B. A variant of the Anderson-Chow estimate}

In [2], Anderson and Chow proved an important estimate for solutions of the parabolic Lichnerowicz equation. In this appendix, we state a variant of that estimate which is due to Kyeongsu Choi.

TheOREM B.1. (Choi) There exists a large constant $C_{\#} \geqslant 10$ and a small positive constant $c_{\#}$ such that the following holds. Let $(M, g(t))$ be a solution to the Ricci flow in dimension 3 with non-negative Ricci curvature, let $h(t)$ be a 1-parameter family of symmetric $(0,2)$-tensors satisfying the parabolic Lichnerowicz equation

$$
\frac{\partial}{\partial t} h(t)=\Delta_{L, g(t)} h(t)
$$

and let $\varrho$ denote a positive real number. Then,

$$
\left(\frac{\partial}{\partial t}-\Delta-\frac{2}{R-\varrho} D^{i} R D_{i}\right)\left(\exp \left(2 c_{\#} \varrho t\right) \frac{|h|^{2}}{(R-\varrho)^{2}}\right) \leqslant 0
$$

whenever $R \geqslant C_{\#} \varrho$.

In the following, we sketch the proof of Theorem B.1. We assume throughout that $R>\varrho>0$. The computation of Anderson-Chow yields

$$
\left(\frac{\partial}{\partial t}-\Delta-\frac{2}{R-\varrho} D^{i} R D_{i}\right) \frac{|h|^{2}}{(R-\varrho)^{2}}=-\frac{2}{(R-\varrho)^{4}}\left|(R-\varrho) D_{i} h_{j k}-D_{i} R h_{j k}\right|^{2}-\frac{4 S}{(R-\varrho)^{3}},
$$

where

$$
S:=-2(R-\varrho)\langle\text { Ric }, h\rangle \operatorname{tr}(h)+2(R-\varrho)\left\langle\text { Ric }, h^{2}\right\rangle-\frac{1}{2} R(R-\varrho)\left(|h|^{2}-\operatorname{tr}(h)^{2}\right)+|h|^{2}|\operatorname{Ric}|^{2}
$$

(cf. $\left[2\right.$, p. 8]). Let us fix a point $p \in M$, and consider an orthonormal basis of $T_{p} M$ with the property that $h$ is diagonal. We denote by $h_{1}, h_{2}$, and $h_{3}$ the diagonal entries of $h$. Moreover, we denote by $r_{1}, r_{2}$, and $r_{3}$ the diagonal entries of Ric. We may assume that $r_{1} \leqslant r_{2} \leqslant r_{3}$. Clearly, $R=r_{1}+r_{2}+r_{3}$ and $\mid$ Ric $\left.\right|^{2} \geqslant r_{1}^{2}+r_{2}^{2}+r_{3}^{2}$. This implies that

$$
2 S \geqslant\left[\begin{array}{lll}
h_{1} & h_{2} & h_{3}
\end{array}\right] A_{\varrho}\left[\begin{array}{l}
h_{1} \\
h_{2} \\
h_{3}
\end{array}\right]
$$

where $A_{\varrho}$ is defined by

$$
A_{\varrho}=\left[\begin{array}{ccc}
2\left(r_{1}^{2}+r_{2}^{2}+r_{3}^{2}\right) & (\varrho-R)\left(r_{1}+r_{2}-r_{3}\right) & (\varrho-R)\left(r_{3}+r_{1}-r_{2}\right) \\
(\varrho-R)\left(r_{1}+r_{2}-r_{3}\right) & 2\left(r_{1}^{2}+r_{2}^{2}+r_{3}^{2}\right) & (\varrho-R)\left(r_{2}+r_{3}-r_{1}\right) \\
(\varrho-R)\left(r_{3}+r_{1}-r_{2}\right) & (\varrho-R)\left(r_{2}+r_{3}-r_{1}\right) & 2\left(r_{1}^{2}+r_{2}^{2}+r_{3}^{2}\right)
\end{array}\right] .
$$


We claim that the matrix $A_{\varrho}$ is positive definite. In order to prove this, we use Sylvester's criterion. The first minor is clearly positive. The second minor satisfies

$$
\begin{aligned}
4\left(r_{1}^{2}+r_{2}^{2}+r_{3}^{2}\right)^{2}-(\varrho-R)^{2}\left(r_{1}+r_{2}-r_{3}\right)^{2} & \geqslant 4\left(r_{1}^{2}+r_{2}^{2}+r_{3}^{2}\right)^{2}-R^{2}\left(r_{1}+r_{2}-r_{3}\right)^{2} \\
& =4\left(r_{1}^{2}+r_{2}^{2}+r_{3}^{2}\right)^{2}-\left(\left(r_{1}+r_{2}\right)^{2}-r_{3}^{2}\right)^{2}>0,
\end{aligned}
$$

where in the last step we have used the inequality

$$
-2\left(r_{1}^{2}+r_{2}^{2}+r_{3}^{2}\right)<\left(r_{1}+r_{2}\right)^{2}-r_{3}^{2}<2\left(r_{1}^{2}+r_{2}^{2}+r_{3}^{2}\right) .
$$

Finally, we consider the third minor of $A_{\varrho}$. Expanding $\operatorname{det} A_{\varrho}$ in powers of $\varrho$ gives

$$
\begin{gathered}
\operatorname{det} A_{\varrho} \geqslant \operatorname{det} A_{0}+4 \varrho R\left(r_{1}^{2}+r_{2}^{2}+r_{3}^{2}\right)\left[\left(r_{1}+r_{2}-r_{3}\right)^{2}+\left(r_{2}+r_{3}-r_{1}\right)^{2}+\left(r_{3}+r_{1}-r_{2}\right)^{2}\right] \\
+6 \varrho R^{2}\left(r_{1}+r_{2}-r_{3}\right)\left(r_{2}+r_{3}-r_{1}\right)\left(r_{3}+r_{1}-r_{2}\right)-C \varrho^{2} R^{4}-C \varrho^{3} R^{3} .
\end{gathered}
$$

By work of Anderson-Chow, $\operatorname{det} A_{0} \geqslant 0$ (see [2, pp. 10-11]). Moreover,

$$
R\left(r_{1}+r_{2}-r_{3}\right)=\left(r_{1}+r_{2}\right)^{2}-r_{3}^{2} \geqslant-r_{3}^{2}
$$

and

$$
0 \leqslant\left(r_{2}+r_{3}-r_{1}\right)\left(r_{3}+r_{1}-r_{2}\right) \leqslant \frac{1}{2}\left[\left(r_{2}+r_{3}-r_{1}\right)^{2}+\left(r_{3}+r_{1}-r_{2}\right)^{2}\right] .
$$

This implies that

$$
R\left(r_{1}+r_{2}-r_{3}\right)\left(r_{2}+r_{3}-r_{1}\right)\left(r_{3}+r_{1}-r_{2}\right) \geqslant-\frac{1}{2} r_{3}^{2}\left[\left(r_{2}+r_{3}-r_{1}\right)^{2}+\left(r_{3}+r_{1}-r_{2}\right)^{2}\right] .
$$

Putting these facts together, we obtain

$$
\begin{gathered}
\operatorname{det} A_{\varrho} \geqslant 4 \varrho R\left(r_{1}^{2}+r_{2}^{2}+r_{3}^{2}\right)\left[\left(r_{1}+r_{2}-r_{3}\right)^{2}+\left(r_{2}+r_{3}-r_{1}\right)^{2}+\left(r_{3}+r_{1}-r_{2}\right)^{2}\right] \\
-3 \varrho R r_{3}^{2}\left[\left(r_{2}+r_{3}-r_{1}\right)^{2}+\left(r_{3}+r_{1}-r_{2}\right)^{2}\right]-C \varrho^{2} R^{4}-C \varrho^{3} R^{3} .
\end{gathered}
$$

Hence, we can find a large constant $C_{\#} \geqslant 10$ and a small positive constant $c$ with the property that $\operatorname{det} A_{\varrho} \geqslant c \varrho R^{5}>0$ whenever $R \geqslant C_{\#} \varrho$. By Sylvester's criterion, the matrix $A_{\varrho}$ is positive definite whenever $R \geqslant C_{\#} \varrho$. Moreover, the largest eigenvalue of $A_{\varrho}$ is bounded by $C R^{2}$ whenever $R>\varrho$. Since $\operatorname{det} A_{\varrho} \geqslant c \varrho R^{5}$, it follows that the smallest eigenvalue of $A_{\varrho}$ is greater than $c \varrho R$ whenever $R \geqslant C_{\#} \varrho$.

To summarize, we have shown that there exists a small positive constant $c_{\#}$ such that $2 S \geqslant c_{\#} \varrho R|h|^{2}$ whenever $R \geqslant C_{\#} \varrho$. Putting these facts together, we conclude that

$$
\left(\frac{\partial}{\partial t}-\Delta-\frac{2}{R-\varrho} D^{i} R D_{i}\right) \frac{|h|^{2}}{(R-\varrho)^{2}} \leqslant-\frac{4 S}{(R-\varrho)^{3}} \leqslant-2 c_{\#} \varrho \frac{|h|^{2}}{(R-\varrho)^{2}}
$$

whenever $R \geqslant C_{\#} \varrho$. From this, the assertion follows. 


\section{References}

[1] Alexakis, S., Chen, D. \& Fournodavlos, G., Singular Ricci solitons and their stability under the Ricci flow. Comm. Partial Differential Equations, 40 (2015), 2123-2172.

[2] Anderson, G. \& Chow, B., A pinching estimate for solutions of the linearized Ricci flow system on 3-manifolds. Calc. Var. Partial Differential Equations, 23 (2005), 1-12.

[3] Angenent, S., Daskalopoulos, P. \& Sesum, N., Uniqueness of two-convex closed ancient solutions to the mean curvature flow. Ann. of Math., 192 (2020), 353-436.

[4] Angenent, S., Daskalopoulos, P. \& ŠEšum, N., Unique asymptotics of ancient convex mean curvature flow solutions. J. Differential Geom., 111 (2019), 381-455.

[5] Angenent, S. \& Knopf, D., An example of neckpinching for Ricci flow on $S^{n+1}$. Math. Res. Lett., 11 (2004), 493-518.

[6] Angenent, S. B., Isenberg, J. \& Knopf, D., Degenerate neckpinches in Ricci flow. J. Reine Angew. Math., 709 (2015), 81-117.

[7] Berline, N., Getzler, E. \& Vergne, M., Heat Kernels and Dirac Operators. Grundlehren Text Editions. Springer, Berlin-Heidelberg, 2004.

[8] Brendle, S., Rotational symmetry of self-similar solutions to the Ricci flow. Invent. Math., 194 (2013), 731-764.

[9] Brendle, S. \& Choi, K., Uniqueness of convex ancient solutions to mean curvature flow in $\mathbb{R}^{3}$. Invent. Math., 217 (2019), 35-76.

[10] BRYAnt, R. L., Ricci flow solitons in dimension three with $S O(3)$-symmetries. Unpublished manuscript, 2005. www . math.duke.edu/ bryant/3DRotSymRicciSolitons . pdf.

[11] Chen, B.-L., Strong uniqueness of the Ricci flow. J. Differential Geom., 82 (2009), 363382.

[12] Chen, B.-L. \& ZHu, X.-P., Uniqueness of the Ricci flow on complete noncompact manifolds. J. Differential Geom., 74 (2006), 119-154.

[13] Daskalopoulos, P., Hamilton, R. \& Šešum, N., Classification of ancient compact solutions to the Ricci flow on surfaces. J. Differential Geom., 91 (2012), 171-214.

[14] Hamilton, R. S., Three-manifolds with positive Ricci curvature. J. Differential Geom., 17 (1982), 255-306.

[15] - Eternal solutions to the Ricci flow. J. Differential Geom., 38 (1993), 1-11.

[16] - The Harnack estimate for the Ricci flow. J. Differential Geom., 37 (1993), 225-243.

[17] - The formation of singularities in the Ricci flow, in Surveys in Differential Geometry, Vol. II (Cambridge, MA, 1993), pp. 7-136. Int. Press, Cambridge, MA, 1995.

[18] Kleiner, B. \& LotT, J., Singular Ricci flows I. Acta Math., 219 (2017), 65-134.

[19] Merle, F. \& ZaAG, H., Optimal estimates for blowup rate and behavior for nonlinear heat equations. Comm. Pure Appl. Math., 51 (1998), 139-196.

[20] Morgan, J. \& Tian, G., Ricci Flow and the Poincaré Conjecture. Clay Math. Monogr., 3. Amer. Math. Soc. Providence, RI; Clay Mathematics Institute, Cambridge, MA, 2007.

[21] Perelman, G., The entropy formula for the Ricci flow and its geometric applications. Unpublished manuscript, 2002. arXiv:0211159 [math.DG].

[22] — Ricci flow with surgery on 3-manifolds. Unpublished manuscript, 2003. arXiv: 0303109 [math.DG].

[23] Topping, P., Lectures on the Ricci Flow. London Math. Soc. Lecture Note Ser., 325. Cambridge Univ. Press, Cambridge, 2006.

[24] YE, R., On the l-function and the reduced volume of Perelman. I. Trans. Amer. Math. Soc., 360 (2008), 507-531.

[25] Zhang, Y., On three-dimensional type I $\kappa$-solutions to the Ricci flow. Proc. Amer. Math. Soc., 146 (2018), 4899-4903. 
Simon Brendle

Department of Mathematics Columbia University

2990 Broadway

New York, NY 10027

U.S.A.

simon.brendle@columbia.edu

Received January 31, 2019 